INT-PUB-02-49

\title{
A Complete Basis for Power Suppressed Collinear-Ultrasoft Operators
}

\author{
Dan Pirjol ${ }^{1}$ and Iain W. Stewart ${ }^{2}$ \\ ${ }^{1}$ Department of Physics and Astronomy, The Johns Hopkins University, \\ Baltimore, MD 21218* \\ ${ }^{2}$ Institute for Nuclear Theory, University of Washington, Seattle, WA $98195^{\dagger}$
}

\begin{abstract}
We construct operators that describe power corrections in mixed collinear-ultrasoft processes in QCD. We treat the ultrasoft-collinear Lagrangian to $\mathcal{O}\left(\lambda^{2}\right)$, and heavy-to-light currents involving collinear quarks to $\mathcal{O}(\lambda)$ including new three body currents. A complete gauge invariant basis is derived which has a full reduction in Dirac structures and is valid for matching at any order in $\alpha_{s}$. The full set of reparameterization invariance (RPI) constraints are included, and are found to restrict the number of parameters appearing in Wilson coefficients and rule out some classes of operators. The QCD ultrasoft-collinear Lagrangian has two $\mathcal{O}\left(\lambda^{2}\right)$ operators in its gauge invariant form. For the $\mathcal{O}(\lambda)$ heavy-to-light currents there are $(4,4,14,14,21)$ subleading (scalar, pseudo-scalar, vector, axial-vector, tensor) currents, where $(1,1,4,4,7)$ have coefficients that are not determined by RPI. In a frame where $v_{\perp}=0$ and $n \cdot v=1$ the total number of currents reduces to $(2,2,8,8,13)$, but the number of undetermined coefficients is the same. The role of these operators and universality of jet functions in the factorization theorem for heavy-to-light form factors is discussed.
\end{abstract}

\footnotetext{
* Electronic address: dpirjol@pha.jhu.edu

$\dagger$ Electronic address: iain@phys.washington.edu
} 


\section{INTRODUCTION}

The soft-collinear effective theory (SCET) constructed in [1, 2, 3, 4] offers a systematic description of processes involving energetic particles. It has an expansion in a small parameter $\lambda \sim p_{\perp} / Q$, where $p_{\perp}$ is a typical transverse momenta and $Q$ the large energy scale. Hard exclusive and inclusive processes in QCD are usually described using the powerful techniques of QCD factorization and light-cone expansions [5, [6]. SCET encompasses and extends these frameworks, and in particular allows a model independent description of effects caused by the interplay between energetic collinear particles and soft particles beyond leading order in the power expansion. These effects can be described in a rigorous way based solely on QCD, but are not included in purely collinear expansions. The study of operators that describe these mixed collinear-ultrasoft (collinear-usoft) effects is the purpose of this paper. For recent applications of SCET in hard scattering processes and B-decays see Refs. [7, 8, 9, 10, 11, 12, 13, 15].

Since our focus is on mixed collinear-usoft interactions, we consider collinear quark fields $\xi_{n, p}$, collinear gluon fields $A_{n, p}^{\mu}$, usoft heavy quark fields $h_{v}$, usoft light quark fields $q_{u s}$, and usoft gluons $A_{u s}^{\mu}$. (We follow the notation in Refs. 22, 3], but for simplicity will often suppress the momentum label $p$ on the collinear fields.) These degrees of freedom can interact in a local manner in Lagrangians and currents. This is in contrast with collinear-soft couplings whose interactions are mediated by offshell fluctuations [4], and appear in external operators. We comment on collinear-soft interactions at the end of the paper.

The derivation of the leading order collinear quark and gluon Lagrangians $\mathcal{L}_{\xi \xi}^{(0)}$ and $\mathcal{L}_{c g}^{(0)}$ can be found in Ref. [2, 4], and a description of the gauge symmetries of SCET can be found in Refs. 3, 4]. For details on power counting we refer to Ref. 12]. The heavy-to-light currents at large energy, $J_{h l}$, were derived to leading order in Ref. 2], including one-loop matching for all the Wilson coefficients. The running of these Wilson coefficients was considered in Refs. [1, 2].

In the context of the SCET, power suppressed corrections were first considered in Ref. [10], and the $\mathcal{O}(\lambda)$ suppressed currents $J_{h l}$ and collinear quark Lagrangians were derived. The authors showed that a reparameterization invariance (RPI) uniquely fixes the Wilson coefficients of their subleading currents and Lagrangian in terms of the leading order coefficients. ${ }^{1}$ In Ref. 11] the RPI of SCET was extended to the most general three classes (I,II,III), and the multipole expansion of the collinear quark Lagrangian was treated to higher orders in $\lambda$ and were shown not to receive anomalous dimensions. In Ref. [12] the presence of additional $\mathcal{O}(\lambda)$ heavy-to-light currents was pointed out that were missing in Ref. [10].

The study of power corrections in SCET was continued in Ref. [13] and several important results were obtained for mixed usoft-collinear operators. In particular the mixed usoftcollinear quark Lagrangian $\mathcal{L}_{\xi q}$ was first considered and was derived to $\mathcal{O}\left(\lambda, \lambda^{2}\right)$ working at tree level, but to all orders in attachments of $\bar{n} \cdot A_{n} \sim \lambda^{0}$ gluon fields. In a similar fashion heavy-to-light currents were derived to $\mathcal{O}\left(\lambda^{2}\right)$, and linear combinations of currents that are

\footnotetext{
${ }^{1}$ A similar application of Lorentz invariance was used to derive constraints on the form of higher-twist contributions to structure functions in deep inelastic scattering in 16]. For this case, invariance under changes in the light-cone vector $\bar{n}_{\mu}$ was used to derive constraints on matrix elements $\left\langle p\left|T \bar{\psi}(0) \Gamma \psi\left(\lambda \bar{n}_{\mu}\right)\right| p\right\rangle$.
} 
invariant under the three types of RPI were identified. It was also shown that the operators in $\mathcal{L}_{\xi q}$ are not renormalized based on an analysis of arbitrary $N$-loop diagrams in the hard region of QCD. The mixed usoft-collinear quark Lagrangian $\mathcal{L}_{\xi q}$ was extended to a gauge invariant form with covariant derivatives in Ref. 14].

The purpose of the present paper is to answer some open questions regarding our knowledge of the power suppressed usoft-collinear Lagrangian and heavy-to-light currents. This includes the number of $J_{h l}$ currents at $\mathcal{O}(\lambda)$, since even at tree level the full reduction of Dirac structures has not yet been implemented. For both $J_{h l}$ and $\mathcal{L}_{\xi q}$ we also construct a complete basis which is valid for matching at any order in $\alpha_{s}$, and therefore includes all operators that can be induced by radiative corrections or operator mixing. We work in the most general possible frame throughout (eg. allowing $v_{\perp} \neq 0, v \cdot n \neq 1$ ), and consider all the restrictions from RPI including the transformation of Wilson coefficients. Finally, we include the mixed usoft-collinear pure glue Lagrangian beyond LO (which follows from an extension of work in Refs. [4, 11]). The above results are obtained by considering the full implications of RPI, and including all possible operators allowed from collinear gauge invariance, power counting, and the reduction of Dirac structures from the effective theory fields. ${ }^{2}$

For the heavy-to-light currents at $\mathcal{O}(\lambda)$ an important result we find is a new type of "three-body" currents, which have not been previously considered in the literature. ${ }^{3}$ In Refs. 10, 12, 13] the attention was restricted to SCET operators of two-body type $J=$ $(\bar{\xi} \ldots W)\left(h_{v}\right)$, where the two products in parenthesis are collinear gauge invariant, and the ellipses denote combinations of collinear derivatives. Beyond tree level but at the same order in $\lambda$, we find that three-body structures can appear for some of the currents, having the form $\mathcal{J}=(\bar{\xi} \ldots W)\left(W^{\dagger} \ldots W\right)\left(h_{v}\right)$ with three collinear gauge invariant factors. We show the RPI can be used to determine for which currents this happens. We also show that RPI greatly restricts the form of the three-body operators, so that they always involve a collinear gluon field strength. The two-body operators have hard Wilson coefficients which are functions of a single parameter $C\left(\omega_{1}\right)$, while the new three-body operators have two parameter coefficients $C\left(\omega_{1}, \omega_{2}\right)$. Analogous three-body structures could appear in the usoft-collinear Lagrangian $\mathcal{L}_{\xi q}$ at higher orders in perturbation theory, however using constraints from symmetries of SCET we prove that this does not occur.

Our results are relevant to the study of decay channels for $B$ mesons which involve energetic hadrons in the final state. For instance, the results derived in this paper are necessary ingredients in the factorization formula for heavy-to-light form factors proven in Ref. [15] (for earlier work on factorization in heavy-to-light form factors see Ref. 18, 19],

\footnotetext{
${ }^{2}$ Note that in deriving the complete basis for $J_{h l}$ we restrict ourselves to $\mathcal{O}(\lambda)$ which is one order less than the order to which the tree-level matching results are known from Ref. 13]. We treat $\mathcal{L}_{\xi q}$ to $\mathcal{O}\left(\lambda^{2}\right)$, and give a detailed account of how the gauge invariant form in Ref. [15] was derived. In cases where our results are restricted to those in Refs. 10, 13] we find agreement, as discussed in more detail in the body of the paper. The results derived here are sufficient for the proof of a factorization theorem for heavy-to-light form factors to all orders in $\alpha_{s}$ and leading order in $1 / Q$ [15].

${ }^{3}$ In the final stages of this paper, Ref. [17] appeared where soft-collinear light-to-light currents are considered. Although different from the usoft-collinear heavy-to-light case studied here, we note that 3-body currents were also found. Further remarks are left to a note added at the end.
} 
and for results from QCD sum rules see Refs. [20]). The factorization theorem is valid to all orders in $\alpha_{s}$ and leading order in $1 / Q, Q=\left\{m_{B}, E\right\}$, and separates contributions from the scales $p^{2} \sim Q^{2}, p^{2} \sim Q \Lambda$, and $p^{2} \sim \Lambda^{2}$, where $\Lambda$ is a hadronic scale. It states that a generic form factor can be split into two types of contributions $F=f^{\mathrm{F}}(Q)+f^{\mathrm{NF}}(Q)$ where [15]

$$
\begin{aligned}
f^{F}(Q)= & N_{0} \int_{0}^{1} d z \int_{0}^{1} d x \int_{0}^{\infty} d r_{+} T\left(z, Q, \mu_{0}\right) \\
& \times J\left(z, x, r_{+}, Q, \mu_{0}, \mu\right) \phi_{M}(x, \mu) \phi_{B}\left(r_{+}, \mu\right), \\
f^{\mathrm{NF}}(Q)= & C_{k}(Q, \mu) \zeta_{k}^{M}(Q, \mu),
\end{aligned}
$$

$N_{0}=f_{B} f_{M} m_{B} /\left(4 E^{2}\right)$, and the two terms both scale as $1 / Q^{3 / 2}$. This scaling is model independent and is in agreement with that derived from QCD Sum Rules [21]. In Eq. (11) $\phi_{M}$ and $\phi_{B}=\phi_{B}^{ \pm}$are standard nonperturbative light-cone distribution amplitudes, c.f. [19, 22]. The hard coefficients $C_{k}$ and $T$ can be calculated in an expansion in $\alpha_{s}(Q)$ and are simply related to the Wilson coefficients of the $\mathcal{O}\left(\lambda^{0}, \lambda^{1}\right)$ current operators $J_{h l}$. The jet function $J$ is dominated by momenta $p^{2} \simeq Q \Lambda$. If we wish to expand in $\alpha_{s}(\sqrt{Q \Lambda})$ then using the techniques developed in Ref. [15], $J$ is calculable in terms of time-ordered products of the $\mathrm{SCET}_{\mathrm{I}}$ operators $J_{h l}$ and $\mathcal{L}_{\xi q}$ that we study here. At tree-level [ie. $\mathcal{O}\left(\alpha_{s}(\sqrt{Q \Lambda})^{1} \alpha_{s}(Q)^{0}\right)$ ] one finds that $J$ contains a $\delta(z-x)$, and in ratios of form factors the results for $f^{F}$ then agree with terms computed in Ref. [19]. The $z$ dependence first shows up at $\mathcal{O}\left(\alpha_{s}^{2}\right)$ as does possible dependence on $\phi_{B}^{-}$. However, as we show in section VI] it is possible to absorb the $\phi_{B}^{-}$terms into a redefinition of the $\zeta_{k}^{M}$ to all orders in perturbation theory.

The factorization formula provides a clean separation of the "soft" non-factorizable (NF) contributions and "hard" factorizable (F) terms without double counting. It also gives us a procedure to systematically improve the predictions to any order in perturbation theory at leading order in $1 / Q$. The value of $T$ and $C_{k}$ can depend on which heavy-to-light process we consider, whereas $\phi_{M}$ and $\phi_{B}^{ \pm}$are universal functions. The $\zeta_{k}$ 's are also universal since only a $\zeta^{M}(E)$ appears for decays to pseudoscalars $M$, and a $\zeta_{\perp}^{M}(E)$ and $\zeta_{\|}^{M}(E)$ appear if $M$ is a vector meson. The jet functions $J$ are common among certain classes of form factors and also do not depend on the precise state (eg. $\pi$ or $\eta$ ). The $f^{N F}$ terms satisfy the socalled large energy form factor relations 15/, as expected from the prior loose definitions of these terms as "soft" contributions [2, 19, 23]. ${ }^{4}$ Note that we have not bothered to separate $p^{2} \sim Q \Lambda$ and $p^{2} \sim \Lambda^{2}$ fluctuations in the $\zeta_{k}^{M}$ functions, since it is not clearly beneficial phenomenologically. The factorization theorem does tell us that $\zeta_{k}^{M} \sim(\Lambda / Q)^{3 / 2}$, however it does not distinguish between factors of $m_{b}$ and $E$ in this $Q^{-3 / 2}$. It also does not numerically favor the $f^{F}$ or $f^{N F}$ term, for instance it is possible that the leading $\alpha_{s}(\sqrt{Q \Lambda})$ in $J$ is compensated by an analogous factor in $\zeta_{k}^{M}$.

We start in Section II by reviewing the general constraints imposed on SCET operators following from collinear gauge invariance, spin structure reduction, and reparameterization invariance. In Section III we study the implications of these predictions for the subleading

\footnotetext{
${ }^{4}$ These relations were first derived in Ref. 23] using LEET [24]. However for studying energetic hadrons with QCD the LEET framework is known to be inconsistent [1, 25], for instance it does not bind an energetic quark-antiquark pair into a meson in heavy-to-light decays [26].
} 
usoft-collinear Lagrangian $\mathcal{L}_{\text {uc }}$. In Section IV we present detailed results for SCET currents. Using the example of the scalar current as the pedagogical example, we demonstrate the construction of the complete basis of $O(\lambda)$ operators contributing to the weak currents, which closes under RPI transformations. Explicit results are then also derived for the pseudo-scalar, vector, axial-vector, and tensor heavy-to-light currents to $\mathcal{O}(\lambda)$. In Section $\mathrm{V}$ we summarize the one-loop matching results for the currents, give explicit results for $\mathcal{L}_{\xi q}$ Feynman rules, and discuss the basis of currents in the particular frame $v_{\perp}=0, n \cdot v=1$.

\section{OPERATOR CONSTRAINTS IN SCET}

In this section we briefly review the symmetries and structure of SCET which will be important for our construction of operators. We refer to Refs. [1, 2, 3, 4, 10, 11] for more details.

SCET includes infrared degrees of freedom corresponding to the relevant low energy scales in the problem. These are typically those with momentum that are collinear $p_{c}^{\mu} \sim Q\left(\lambda^{2}, 1, \lambda\right)$, soft $p_{s}^{\mu} \sim Q(\lambda, \lambda, \lambda)$, or ultrasoft (usoft) $p_{u s}^{\mu} \sim Q\left(\lambda^{2}, \lambda^{2}, \lambda^{2}\right)$, where the components here are in a light-cone basis $(+,-, \perp)$. Each type of mode has effective theory quark and gluon fields, which are then organized into operators with a well-defined power counting in $\lambda$. It is convenient to introduce light-cone unit vectors $\left(n_{\mu}, \bar{n}_{\mu}\right)$ satisfying $n^{2}=\bar{n}^{2}=0, n \cdot \bar{n}=2$, in terms of which a vector has components $p^{\mu}=\left(n \cdot p, \bar{n} \cdot p, p_{\perp}^{\mu}\right)$. The couplings of the fields are described by an effective Lagrangian, while the couplings to external sources appear as additional operators or currents. Both the Lagrangian and currents are constructed such that they include constraints from power counting, spin symmetries, and collinear and (u)soft gauge invariance.

The soft-collinear effective theory also contains a kinematical reparameterization invariance symmetry. Lorentz invariance is broken by introducing the vectors $n$ and $\bar{n}$, but is restored order by order in $\lambda$, by requiring invariance of operators under a simultaneous change in $n$ and/or $\bar{n}$ and compensating changes in the effective theory fields. This reparameterization invariance (RPI) symmetry of SCET was first considered in Ref. [10], and was then extended to the most general three classes (I,II,III) of allowed transformations in Ref. [11]. ${ }^{5}$ The three types are defined by the infinitesimal change they induce on the light-cone unit vectors: type-I $\left(n \rightarrow n+\Delta_{\perp}\right)$, type-II $\left(\bar{n} \rightarrow \bar{n}+\varepsilon_{\perp}\right)$, and type-III $(n \rightarrow(1+\alpha) n, \bar{n} \rightarrow(1-\alpha) \bar{n})$. Here $\alpha \sim \varepsilon_{\perp} \sim \lambda^{0}$, while $\Delta_{\perp} \sim \lambda$. It is the analog of the reparameterization invariance of heavy quark effective theory (HQET) under changes in the heavy quark velocity $v$ [28], where $v^{2}=1$. We will use HQET for heavy quark fields [29].

The restrictions we consider for finding the most general set of power suppressed gauge invariant operators are:

\footnotetext{
${ }^{5}$ The nature of Lorentz symmetries on the light-cone are well known [27]. The new point in SCET [10, 11] is that for any collinear process these symmetries are realized in a way that leads to non-trivial restrictions both on operators at a given order in the power counting and between operators at different orders in the power counting.
} 
i) Power counting and gauge invariance which determine what basic building blocks are allowed at the order we are considering.

ii) What auxiliary vectors are available ( $\operatorname{such}$ as $n, \bar{n}, v, \ldots$ ), which can be used to construct the most general set of allowed scalars/tensors/Dirac structures.

iii) Eliminate operators which are redundant by integration by parts, or equations of motion.

iv) Impose type-III reparameterization invariance. If a non-trivial invariant can be formed with the label operators, such as $(n \cdot v \overline{\mathcal{P}})$, then include Wilson coefficients that depends on these quantities.

v) Impose all constraints from type-I and type-II reparameterization invariance.

To impose the five constraints we start by writing minimal sets of independent operators compatible with the general principles in i), ii), and iii). We then require RPI invariance order by order in the $\lambda$ power counting. To do this we found it useful to split the RPI transformations into two categories, those that act within the order we are considering $\delta_{j}^{\left(\lambda^{0}\right)}$, with $j=$ I,II,III, and those which connect operators to one higher order $\delta_{j}^{(\lambda)}$. At leading order the type-II and type-III $\delta_{j}^{\left(\lambda^{0}\right)}$ transformations already provide non-trivial constraints on the allowed form of operators. In contrast the $\delta_{j}^{(\lambda)}$ transformations allow us to derive relations valid to all orders in $\alpha_{s}$ between the Wilson coefficients of operators at different orders in $\lambda$, These relations are similar to the case of RPI in HQET [28, 30], where we note in particular the important relations derived for coefficients of subleading heavy-to-heavy currents in Ref. 31]. We start by summarizing restrictions that follow from collinear gauge invariance and power counting in section $\llbracket \mathrm{A}$, spin structure reductions in section RPI in section $\amalg$ IC.

To separate the momentum scales we follow Ref. [2] and use collinear quark fields $\xi_{n, p}(x)$ (and gluon fields $A_{n, p}^{\mu}(x)$ ) which have momentum labels $p$ for the large components of the collinear momenta, and residual coordinates $x^{\mu} \sim 1 / \lambda^{2}[2]$. Thus, all derivatives on collinear fields are the same size as derivatives on usoft fields, $\partial^{\mu} \sim \lambda^{2}$. This setup implements the multipole expansion in momentum space. Note that our analysis of power corrections differs from Ref. [13] in two ways, the first being that in Ref. [13] the momentum scales were separated by performing the multipole expansion in position space, which however leads to an equivalent formulation. We do find that concise results for the power suppressed corrections are obtained with the momentum space version. Secondly, we derive our basis of operators and implement all symmetry constraints working order by order in the power counting, rather than constructing invariants and then expanding in $\lambda$. This made it simpler to derive a complete gauge invariant basis at the desired order while working in a general frame. 


\section{A. Power Counting and Gauge Invariance}

The SCET is derived from QCD by integrating out fluctuations with $p^{2} \gg Q^{2} \lambda^{2}$, where in typical processes $\lambda=\left(\Lambda_{\mathrm{QCD}} / Q\right)^{k}$ with $k=1$ or $k=1 / 2$. Infrared fluctuations are then described by effective theory fields. A gauge invariant power counting for fields can be fixed by demanding that the kinetic terms in the action are order $\lambda^{0}$. For the collinear fields this gives $\xi_{n} \sim \lambda^{0}$ for the quarks, and $\left(n \cdot A_{n}, \bar{n} \cdot A_{n}, A_{n}^{\perp \mu}\right) \sim\left(\lambda^{2}, \lambda^{0}, \lambda\right)$ for the collinear gluons, $h_{v} \sim q \sim \lambda^{3}$ for usoft quarks, and $A_{u s}^{\mu} \sim \lambda^{2}$ for usoft gluons [1, 2]. Derivatives on these fields count as $\partial^{\mu} \sim \lambda^{2}$. The larger collinear momenta are picked out by introducing label operators $\overline{\mathcal{P}} \sim \lambda^{0}$ and $\mathcal{P}_{\perp}^{\mu} \sim \lambda[\underline{3}]$. For example $\overline{\mathcal{P}} \xi_{n, p}=(\bar{n} \cdot p) \xi_{n, p}$. For notational convenience we define collinear covariant derivatives

$$
i \bar{n} \cdot D_{c}=\overline{\mathcal{P}}+g \bar{n} \cdot A_{n}, \quad i D_{c}^{\perp \mu}=\overline{\mathcal{P}}_{\perp}^{\mu}+g A_{n}^{\perp \mu},
$$

and ultrasoft covariant derivatives

$$
i \bar{n} \cdot D_{u s}=i \bar{n} \cdot \partial+g \bar{n} \cdot A_{u s}, \quad i D_{u s}^{\perp \mu}=i \partial_{\perp}^{\mu}+g A_{u s}^{\perp \mu} .
$$

For the $n^{\mu}$ components, it is only the combination

$$
i n \cdot D=i n \cdot \partial+g n \cdot A_{n}+g n \cdot A_{u s},
$$

that ever appears. In general a derivative without a subscript involves the sum of the collinear and usoft pieces, $D^{\mu}=D_{c}^{\mu}+D_{u s}^{\mu}$, and it is this combination which is RPI invariant [11] (implying that the anomalous dimensions of terms that appear in the multipole expansion are related).

Integrating out the offshell fluctuations builds up a collinear Wilson line, $W$, built out of collinear gluon fields which are not suppressed in the power counting [3]

$$
W=\left[\sum_{\text {perms }} \exp \left(-\frac{g}{\overline{\mathcal{P}}} \bar{n} \cdot A_{n, q}(x)\right)\right],
$$

where the label operators only act on fields inside the square brackets. Up to the important fact that $W$ has been multipole expanded, it is the Fourier transform of a standard position space Wilson line, $W(-\infty, x)$. Factors of $W \sim \lambda^{0}$ can be included in operators without changing the order in the power counting. However, their location is restricted by collinear gauge transformations, $U_{c}$, under which $W \rightarrow U_{c} W[3]$. Since $\overline{\mathcal{P}} \sim \lambda^{0}$ in the power counting the hard Wilson coefficients can be arbitrary functions of the momentum or momenta, $\omega_{i}$, picked out by these operator, $C\left(\omega_{i}, \mu\right)$ 3]. These coefficients can be computed by matching with $\mathrm{QCD}$ at the hard scale $\mu \simeq Q$ and running with the renormalization group.

If we consider a general Wilson coefficient and operator $C \otimes \mathcal{O}$, then the covariant derivative

$$
i \bar{n} \cdot D_{c}=W \overline{\mathcal{P}} W^{\dagger},
$$

so it is always possible to put all the Wilson lines in $\mathcal{O}$ and the dependence on the momenta picked out by $\overline{\mathcal{P}}$ into $C$. We will find it convenient to use the notation

$$
\begin{aligned}
\left(\bar{\xi}_{n} W\right)_{\omega_{1}} & =\left[\bar{\xi}_{n} W \delta\left(\omega_{1}-n \cdot v \overline{\mathcal{P}}^{\dagger}\right)\right] \\
\left(W^{\dagger} D_{c}^{\perp \mu} W\right)_{\omega_{2}} & =\left[W^{\dagger} D_{c}^{\perp \mu} W \delta\left(\omega_{2}-n \cdot v \overline{\mathcal{P}}^{\dagger}\right)\right],
\end{aligned}
$$


where again the label operators do not act outside the square brackets. The factor of $n \cdot v$ is included next to $\overline{\mathcal{P}}$ to make it a type-III RPI invariant. Thus, the momentum labels $\omega_{i}$ do not transform under RPI. The products of fields in Eq. (8) are color singlets under the collinear gauge symmetry, so the momentum labels $\omega_{i}$ are gauge invariant. These products still transform under an usoft gauge transformation $U_{u s}$ as $\left(\bar{\xi}_{n} W\right) \rightarrow\left(\bar{\xi}_{n} W\right) U_{u s}^{\dagger}$ and $\left(W^{\dagger} D_{c}^{\perp \mu} W\right) \rightarrow U_{u s}\left(W^{\dagger} D_{c}^{\perp \mu} W\right) U_{u s}^{\dagger}$. We will elaborate on how RPI affects Wilson coefficients in SCET in subsection $1 \mathrm{C}$ below.

For Lagrangians and currents where the variable $v^{\mu}$ is not available we can not make use of the definitions in Eq. (8). It is still convenient to make use of a similar notation:

$$
\begin{aligned}
\left(\bar{\xi}_{n} W\right)_{z_{1}} & =\left[\bar{\xi}_{n} W \delta\left(z_{1}-\overline{\mathcal{P}}^{\dagger}\right)\right], \\
\left(W^{\dagger} D_{c}^{\perp \mu} W\right)_{z_{2}} & =\left[W^{\dagger} D_{c}^{\perp \mu} W \delta\left(z_{2}-\overline{\mathcal{P}}^{\dagger}\right)\right],
\end{aligned}
$$

where we use the variables $z_{i}$ rather than $\omega_{i}$. Under a type-III transformation the $z_{i}$ transform like $\bar{n}$ so the delta function is homogeneous (and compensated by an integration measure $\left.d z_{i}\right)$.

Using the scalings for fields and derivatives the power counting for an arbitrary diagram, $\lambda^{\delta}$, can be determined entirely from its operators using [12]

$$
\delta=4+\sum_{k}(k-4)\left[V_{k}^{C}+V_{k}^{S}+V_{k}^{S C}\right]+(k-8) V_{k}^{U} .
$$

Here $V_{k}^{C, S, S C, U}$ count the number of order $\lambda^{k}$ operators which have collinear fields, soft fields, both, or neither respectively. For any operator the power of $k$ is derived by adding up the powers of $\lambda$ in its components, so for instance $\bar{\xi}_{n} \not{\hbar}$ in $\cdot D \xi_{n} \sim \lambda^{4}$, counts as $V_{4}^{C}=1$. Since the operators are gauge invariant so is their value of $k$ and also the power counting of any diagram using the result for $\delta$ in Eq. (10). In this paper we focus on operators with $V_{k}^{S}=V_{k}^{S C}=0$.

We have also found it convenient to define additional pure gluon operators. In particular we will use the purely collinear field strength

$$
i g B_{c}^{\perp \mu}=\left[i \bar{n} \cdot D^{c}, i D_{c}^{\perp \mu}\right] .
$$

We will also make use of the mixed tensors

$$
i g n \cdot M=i g n \cdot B_{c}=\left[i \bar{n} \cdot D^{c}, i n \cdot D\right], \quad i g M_{\perp}=\left[i \bar{n} \cdot D^{c}, i \not D_{\perp}^{u s}\right]
$$

In fact the operators $M_{\perp}$ and $n \cdot M$, together with $i g \bar{n} \cdot M=\left[i \bar{n} \cdot D^{c}, i \bar{n} \cdot D^{u s}\right]$ can be combined into a single object closed under usoft Lorentz transformations, which transforms in the desired way under the collinear and usoft gauge symmetries

$$
i g M=\left[i \bar{n} \cdot D^{c}, i \not D^{u s}+\frac{\not h}{2} g n \cdot A_{n}\right] .
$$

Finally the following results for manipulating covariant derivatives on Wilson lines also prove to be useful

$$
\begin{aligned}
& \left(W^{\dagger} i \overleftarrow{D}_{c}^{\perp} W\right)=\left[W^{\dagger} i \overleftarrow{D}_{c}^{\perp} W\right]-\mathscr{P}_{\perp}^{\dagger}=\left[\frac{1}{\overline{\mathcal{P}}} W^{\dagger} i g \mathbb{P}_{c}^{\perp} W\right]-\mathcal{P}_{\perp}^{\dagger} \\
& \left(W^{\dagger} i \overrightarrow{D D}_{c}^{\perp} W\right)=\left[W^{\dagger} \overrightarrow{\not D}_{c}^{\perp} W\right]+\mathscr{P}_{\perp}=\left[\frac{1}{\overline{\mathcal{P}}} W^{\dagger} i g \mathbb{P}_{c}^{\perp} W\right]+\mathscr{P}_{\perp}
\end{aligned}
$$




\section{B. Reduction in Spin Structures}

Collinear quarks and heavy usoft quarks have spinors with only two non-zero components. In four component notation this is encoded in projection formulae for the fields,

$$
P_{n} \xi_{n}=\xi_{n}, \quad P_{v} h_{v}=h_{v},
$$

where $P_{n}=(\not h \not h) / 4$ and $P_{v}=(1+\psi) / 2$. We also define the orthogonal projector $P_{\bar{n}}=(\not h \not h$ ) $/ 4$ where $P_{n}+P_{\bar{n}}=1$. A quark bilinear with a heavy ultrasoft quark and light collinear quark therefore only has four possible non-trivial Dirac structures. On the other hand if the heavy ultrasoft quark is replaced by a massless ultrasoft quark which has a four component spinor then their are eight possible Dirac structures. When generating operators we should be careful not to include redundant Dirac structures. Therefore, it is convenient to have a canonical basis which we can project results onto to check their interdependence. For this purpose we choose the basis

$$
\begin{array}{ll}
\bar{\xi}_{n} \Gamma_{1} h_{v}, & \Gamma_{1}=\left\{\frac{\not h}{2}, \frac{\not h \gamma^{5}}{2}, \gamma_{\perp}^{\mu}\right\}, \\
\bar{\xi}_{n} \Gamma_{2} q_{u s}, & \Gamma_{2}=\left\{1, \frac{\not h}{2}, \gamma^{5}, \frac{\not h \gamma^{5}}{2}, \gamma_{\perp}^{\mu}, \frac{\not h \gamma_{\perp}^{\mu}}{2}\right\} .
\end{array}
$$

Any general Dirac structure can be projected onto a linear combination of terms in these basis with the help of the following formulae

$$
\begin{aligned}
\bar{\xi}_{n} \Gamma h_{v} & =\bar{\xi}_{n} \Gamma_{1} h_{v} \\
\Gamma_{1} & =\frac{\not h}{2} \operatorname{tr}\left[\frac{\not h}{2} P_{\bar{n}} \Gamma P_{v}\right]-\frac{\not h \gamma_{5}}{2} \operatorname{tr}\left[\frac{\not h}{2} \gamma_{5} P_{\bar{n}} \Gamma P_{v}\right]+\gamma_{\perp}^{\mu} \operatorname{tr}\left[\gamma_{\mu}^{\perp} P_{\bar{n}} \Gamma P_{v}\right] .
\end{aligned}
$$

and

$$
\begin{aligned}
\bar{\xi}_{n} \Gamma q_{u s}= & \bar{\xi}_{n} \Gamma_{2} q_{u s}, \\
\Gamma_{2}= & 1 \operatorname{tr}\left[\frac{\not h \not h}{8} \Gamma\right]+\frac{\not h}{2} \operatorname{tr}\left[\frac{\not h}{4} \Gamma\right]+\gamma_{5} \operatorname{tr}\left[\frac{\not h \not h \gamma_{5}}{8} \Gamma\right]-\frac{\not h \gamma_{5}}{2} \operatorname{tr}\left[\frac{\not h \gamma_{5}}{4} \Gamma\right] \\
& +\gamma_{\perp}^{\mu} \operatorname{tr}\left[\frac{\gamma_{\mu}^{\perp} \not h \not h}{8} \Gamma\right]-\frac{\not h \gamma_{\perp}^{\mu}}{2} \operatorname{tr}\left[\frac{\not h \gamma_{\mu}^{\perp}}{4} \Gamma\right] .
\end{aligned}
$$

The number of independent structures is quite logical, for $\bar{\xi}_{n} \Gamma h_{v}$ each field is determined by two-component spinors and there are $2 \times 2=4$ terms in the basis. For $\bar{\xi}_{n} \Gamma q_{u s}$ only the collinear spinor has two-components and there are $2 \times 4=8$ terms in our basis. Our choice of basis in Eq. (17) differs from Ref. [2] where the choice $\Gamma_{1}=\left\{1, \gamma_{5}, \gamma_{\perp}^{\mu}\right\}$ was used, and calculations were given in a frame where $v \cdot n=1$ and $v_{\perp}=0$. When $v^{\mu}$ is kept arbitrary we have found the basis in Eq. (16) is more convenient since it retains its orthonormality in an arbitrary frame.

The projections formulae in Eq. (17) can be used to reduce the possible Dirac structures in constructing a complete basis of operators. It is convenient to define $r_{\perp}^{\mu} \equiv i \epsilon^{\mu \alpha \beta \gamma} n_{\alpha} \bar{n}_{\beta} v_{\gamma}$ and $v_{\perp}^{\mu}=v^{\mu}-n \cdot v \bar{n}^{\mu} / 2-\bar{n} \cdot v n^{\mu} / 2$, since then $v_{\perp}^{\mu}, r_{\perp}^{\mu}, n^{\mu}, \bar{n}^{\mu}$ form a complete vector basis. 
To reduce the Dirac structures we can use relations such as

$$
\begin{aligned}
& \psi_{\perp} \doteq 1-\frac{n \cdot v}{2} \not \vec{n}, \quad \not h \psi_{\perp} \doteq \not{h}-2 \bar{n} \cdot v, \quad \gamma_{\perp}^{\mu} \doteq \gamma_{\mu}-\frac{n \cdot v}{2} \not{n}, \\
& r_{\perp}^{\mu} \gamma_{5} \doteq-2 v_{\perp}^{\mu}+2 \gamma_{\perp}^{\mu}+\not h \gamma_{\perp}^{\mu} n \cdot v, \quad \not h r_{\perp}^{\mu} \gamma_{5} \doteq 2 \not h v_{\perp}^{\mu}-4 \bar{n} \cdot v \gamma_{\perp}^{\mu}-2 \not h \gamma_{\perp}^{\mu} \text {, } \\
& i \epsilon^{\mu \nu \alpha \beta} n_{\alpha} v_{\beta} \frac{\not h \gamma_{5}}{2} \doteq i \sigma_{\mu \nu}-\left(v_{\mu} \gamma_{\nu}-v_{\nu} \gamma_{\mu}\right)-\frac{1}{2}\left(\bar{n}_{\mu} n_{\nu}-n_{\mu} \bar{n}_{\nu}\right)-\frac{\bar{n} \cdot v}{2}\left(n_{\mu} \gamma_{\nu}-n_{\nu} \gamma_{\mu}\right) \\
& +\frac{n \cdot v}{2}\left(\bar{n}_{\mu} \gamma_{\nu}-\bar{n}_{\nu} \gamma_{\mu}\right), \quad \ldots \text {, }
\end{aligned}
$$

where the $\doteq$ indicates that these are only true between the fields in Eq. (17). (The complete set of relations is rather lengthy and is not shown.) The relations in Eq. (19) allow the structures on the left to be traded for those on the right (with more than one iteration in some cases). Using the projection formulae it is straightforward to show that the most general Dirac structure possible for the LO scalar currents are $\{1, \vec{n}\}$, while the vector and axial-vector currents have the basis shown in Eq. (74)), and the tensor currents depend on the basis in Eq. (92)

\section{Reparameterization Invariance}

The decomposition into collinear fields requires introducing two light-like vectors $n$ and $\bar{n}$, such that $n^{2}=\bar{n}^{2}=0$ and $n \cdot \bar{n}=2$. These vectors break five of the six Lorentz generators. This part of the Lorentz symmetry is restored order by order in the power counting by requiring invariance under reparameterization transformations on $n$ and $\bar{n}$ [11]:

$$
\text { (I) }\left\{\begin{array} { l } 
{ n _ { \mu } \rightarrow n _ { \mu } + \Delta _ { \mu } ^ { \perp } } \\
{ \overline { n } _ { \mu } \rightarrow \overline { n } _ { \mu } }
\end{array} \quad \text { (II) } \left\{\begin{array} { l } 
{ n _ { \mu } \rightarrow n _ { \mu } } \\
{ \overline { n } _ { \mu } \rightarrow \overline { n } _ { \mu } + \varepsilon _ { \mu } ^ { \perp } }
\end{array} \quad \text { (III) } \left\{\begin{array}{l}
n_{\mu} \rightarrow(1+\alpha) n_{\mu} \\
\bar{n}_{\mu} \rightarrow(1-\alpha) \bar{n}_{\mu}
\end{array}\right.\right.\right.
$$

where $\Delta^{\perp} \sim \lambda$, while $\varepsilon^{\perp} \sim \alpha \sim \lambda^{0}$. In general one has two options for constructing RPI invariants: i) construct operators out of completely RPI invariant quantities and then expand these in powers of $\lambda$, ii) construct operators order by order in $\lambda$ and transform them to see what linear combinations are invariant, and which operators are ruled out. In this paper we will adopt approach ii), since starting with the most general gauge invariant sets and then reducing them allows us to be confident that we do not miss operators that could arise at any order in perturbation theory.

For our purposes it is convenient to divide the RPI transformations into two subsets, those which include terms within the same order in $\lambda$ denoted by $\delta_{\mathrm{I}}^{\left(\lambda^{0}\right)}, \delta_{\mathrm{II}}^{\left(\lambda^{0}\right)}$, and $\delta_{\mathrm{III}}^{\left(\lambda^{0}\right)}$, and those which cause order $\lambda$ suppressed transformations denoted by $\delta_{\mathrm{I}}^{(\lambda)}$ and $\delta_{\mathrm{II}}^{(\lambda)}$. All typeIII transformations act within the same order in $\lambda$ and it is easy to construct invariants under type-III. We simply need to have the same number of $n$ 's ( $\bar{n}$ 's) in the numerator and denominator, or have products of $n$ times $\bar{n}$. The transformations of type-I and type-II are more involved. From Ref. [1] the transformations that have terms of the same order in $\lambda$ 
are

$$
\begin{array}{ll}
\delta_{\mathrm{I}}^{\left(\lambda^{0}\right)}: & n \cdot D \rightarrow n \cdot D+\Delta^{\perp} \cdot D_{\perp}, \quad D_{\perp}^{\mu} \rightarrow D_{\perp}^{\mu}-\frac{\Delta_{\perp}^{\mu}}{2} \bar{n} \cdot D, \\
\delta_{\mathrm{II}}^{\left(\lambda^{0}\right)}: & \bar{n}^{\mu} \rightarrow \bar{n}^{\mu}+\varepsilon_{\perp}^{\mu}, \quad D_{\perp}^{\mu} \rightarrow D_{\perp}^{\mu}-\frac{n^{\mu}}{2} \varepsilon_{\perp} \cdot D_{\perp}, \quad \gamma_{\perp}^{\mu} \rightarrow \gamma_{\perp}^{\mu}-\frac{n^{\mu}}{2} \notin_{\perp}-\frac{\varepsilon_{\perp}^{\mu}}{2} \hbar,
\end{array}
$$

and the transformations that start one power down in $\lambda$ include

$$
\begin{aligned}
\delta_{\mathrm{I}}^{(\lambda)}: \quad n^{\mu} \rightarrow n^{\mu}+\Delta_{\perp}^{\mu}, \quad D_{\perp}^{\mu} \rightarrow D_{\perp}^{\mu}-\frac{\bar{n}^{\mu}}{2} \Delta^{\perp} \cdot D_{\perp}, \quad \bar{\xi}_{n} \rightarrow \bar{\xi}_{n}\left(1+\frac{\not h \phi_{\perp}}{4}\right), \\
\gamma_{\perp}^{\mu} \rightarrow \gamma_{\perp}^{\mu}-\frac{\Delta_{\perp}^{\mu} \not h}{2}-\frac{\bar{n}^{\mu}}{2} \phi_{\perp}, \\
\delta_{\mathrm{II}}^{(\lambda)}: \quad \bar{n} \cdot D \rightarrow \bar{n} \cdot D+\varepsilon_{\perp} \cdot D_{\perp}, \quad D_{\perp}^{\mu} \rightarrow D_{\perp}^{\mu}-\frac{\varepsilon_{\perp}^{\mu}}{2} n \cdot D, \\
\bar{\xi}_{n} \rightarrow \bar{\xi}_{n}\left(1+\overleftarrow{D D}_{\perp} \frac{1}{\bar{n} \cdot \overleftarrow{D}} \frac{\ddagger_{\perp}}{2}\right), \quad \mathcal{W} \rightarrow\left[\left(1-\frac{1}{\bar{n} \cdot D} \varepsilon^{\perp} \cdot D^{\perp}\right) \mathcal{W}\right] .
\end{aligned}
$$

Here $\mathcal{W}$ is the RPI completed $W$, and is the Fourier transform with respect to $y$ of a position space Wilson line involving $\left(\bar{n} \cdot A_{n}+\bar{n} \cdot A_{u s}\right)(s \bar{n}+x)$ taken from $s=-\infty$ to y [13]. When expanded in $\lambda, \mathcal{W}=W+\mathcal{O}\left(\lambda^{2}\right)$, where $W$ involves only the $\bar{n} \cdot A_{n}$ field as in Eq. (6).

If we start by considering LO operators then they must be invariant under the $\delta^{\left(\lambda^{0}\right)}$ transformations in Eq. (21) all by themselves. The $\delta^{(\lambda)}$ transformations of the LO terms connect them to NLO operator's $\delta^{\left(\lambda^{0}\right)}$ transformations. Since in the collinear sector only $\delta^{\left(\lambda^{0}, \lambda^{1}\right)}$ terms exist this pattern repeats at all higher orders in the power counting. Note that here we will not need to consider HQET RPI under the velocity $v^{\mu}$. Since the transformation $v^{\mu} \rightarrow v^{\mu}+\Delta_{v}^{\mu}$ where $\Delta_{v}^{\mu} \sim \Lambda_{\mathrm{QCD}} / Q \sim \lambda^{2}$, this type of RPI only needs to be taken into account at one-higher order than the order we are working. The combined SCET and HQET RPI transformations were used in the $\mathcal{O}\left(\lambda^{2}\right)$ analysis of $J_{h l}$ in Ref. 13].

Finally we consider a new feature of RPI in SCET, namely how Wilson coefficients are affected by reparameterization invariance. Our analysis is similar in spirit to Ref. 31], where heavy-to-heavy HQET currents with coefficients depending on the change in velocity, $C\left(v \cdot v^{\prime}\right)$, were analyzed. If we adopt the view of building invariants at all orders in $\lambda$ then the coefficients in SCET must also be functions of invariants, such as operators like

$$
\bar{\Psi}_{n} C(-i \overleftarrow{D} \cdot \mathcal{V}) \Gamma \mathcal{H}_{v}
$$

where $\Psi_{n}, \mathcal{H}_{v}$ are invariants including the quark fields $\xi_{n}, h_{v}$, and $\mathcal{V}^{\mu}$ is the RPI version of the velocity $v^{\mu}$ [28]. When expanded in $\lambda$ the leading term involving the covariant derivative in $C$ can be traded for $W$ and $\overline{\mathcal{P}}$ using Eq. (7), $C\left(-i n \cdot v \bar{n} \cdot \overleftarrow{D}_{c}\right)=W C\left(n \cdot v \overline{\mathcal{P}}^{\dagger}\right) W^{\dagger}$. Here we will use the opposite but equivalent arrangement of starting with a current that is leading order in $\lambda$,

$$
\bar{\xi}_{n} W C\left(n \cdot v \overline{\mathcal{P}}^{\dagger}\right) h_{v}
$$

and then determining how both the operators and coefficient transform under RPI. We then determine which structures are required at one higher order in $\lambda$ to cancel this change, and which allowed higher order structures are left unconstrained. 


\section{COLLINEAR-ULTRASOFT LAGRANGIAN}

In this section we discuss the mixed ultrasoft-collinear Lagrangians to $\mathcal{O}\left(\lambda^{2}\right)$. These actions are power suppressed [7], and start at $\mathcal{O}(\lambda)$ [13]. In section III we consider the derivation from integrating out components of the full theory field, which gives a tree level derivation of the action (for further explanation of this approach see Refs. [2, 4, 13]). In Ref. 13. this procedure was used to derive a form for the mixed ultrasoft-collinear quark Lagrangian, but a manifestly gauge invariant form was not determined. In Ref. [14] the analysis was extended to give manifestly gauge invariant operators in terms of covariant derivatives. In section IIIA we review the details of how a derivation of a gauge invariant form of the action was carried out in Ref. [15] where the result is purely in terms of field strengths.

However, since the analysis in section $\llbracket \mathrm{A}$ is only valid at tree level, it misses i) nontrivial Wilson coefficients in the tree level operators, and ii) new operators whose coefficients can have zero tree-level matching. In Ref. [13] point i) was addressed and it was shown diagrammatically that no non-trivial Wilson coefficients are generated. However, point ii) has not yet been addressed, so additional operators could still be induced by matching at some higher order in perturbation theory. In section IIB we show that both points i) and ii) can be simultaneously solved by using the full set of symmetries of SCET when constructing operators. We also extend the derivation to the mixed usoft-collinear pure gluon sector.

\section{A. Matching for $\mathcal{L}_{u c}$ at tree level, but all orders in $\bar{n} \cdot A_{n}$ gluons}

In this subsection we discuss in detail the matching calculation for the mixed usoftcollinear quark Lagrangian 15]. The part of our discussion from Eq. (26) to (30) follows Ref. [13], but with our momentum space notation. We start with the action $\mathcal{L}=\bar{\psi} i \not D \psi$ and decompose it with SCET fields

$$
\begin{aligned}
\mathcal{L}= & \bar{\xi}_{n} \frac{\not h}{2} i n \cdot D \xi_{n}+\bar{\xi}_{n} i \not D^{\perp} \xi_{\bar{n}}+\bar{\xi}_{n} g A_{c} q_{u s}+\bar{q}_{u s} g A_{c} \xi_{n}+\bar{q}_{u s} g A_{c} \xi_{\bar{n}}+\bar{q}_{u s} i \not D_{u s} q_{u s} \\
& +\left[\bar{\xi}_{\bar{n}} i \not D^{\perp} \xi_{n}+\bar{\xi}_{\bar{n}} \frac{\not h}{2} i \bar{n} \cdot D \xi_{\bar{n}}+\bar{\xi}_{\bar{n}} g A_{c} q_{u s}\right],
\end{aligned}
$$

where the $D$ is usoft plus collinear, $D_{c}$ is purely collinear, and collinear momentum conservation has been enforced. Varying with respect to $\bar{\xi}_{\bar{n}}$ gives an equation of motion to eliminate this field from the term in square brackets

$$
\xi_{\bar{n}}=-\frac{1}{i \bar{n} \cdot D} \frac{\not h}{2}\left[i \not D_{\perp} \xi_{n}+g A_{n} q_{u s}\right], \quad \bar{\xi}_{\bar{n}}=\left[\bar{q}_{u s} g A_{n}-\bar{\xi}_{n} i \overleftarrow{D_{\perp}}\right] \frac{\not h}{2} \frac{1}{i \bar{n} \cdot \overleftarrow{D}}
$$

Plugging this into Eq. (26) and expanding we find that the two collinear quark terms exactly reproduce terms in the gauge invariant multipole expanded action in Ref. [11]. ${ }^{6}$ Using

\footnotetext{
${ }^{6}$ Note that in QED the $\mathcal{O}(\lambda)$ pure collinear quark Lagrangian can be written in terms of $F_{\mu \nu}$ 13. With the momentum space multipole expansion this manipulation is not necessary to achieve a gauge invariant result [33, 34].
} 
Eq. (10) the terms with two ultrasoft quarks and $\geq 2$ collinear gluons first show up at $\delta=3$, ie. $\mathcal{O}\left(\lambda^{3}\right)$ [13], and are therefore neglected. The mixed usoft-collinear quark terms are

$$
\mathcal{L}_{\xi q}=\left[\bar{\xi}_{n} g A_{n} q_{u s}+\bar{\xi}_{n} \frac{\not h}{2} i \not D_{\perp} \frac{1}{i \bar{n} \cdot D} g A_{n} q_{u s}\right]+\left[\bar{q}_{u s} g A_{n} \xi_{n}+\bar{q}_{u s} g A_{n} \frac{1}{i \bar{n} \cdot D} i \not D_{\perp} \frac{\not h}{2} \xi_{n}\right] .
$$

Taking Eq. (28) and expanding to second order in $\lambda$ gives

$$
\begin{aligned}
\mathcal{L}_{\xi q}^{(1)} & =\bar{\xi}_{n}\left(g A_{\perp}^{c}-i \not D_{\perp} \frac{1}{i \bar{n} \cdot D_{c}} g \bar{n} \cdot A_{c}\right) q_{u s}+\text { h.c. } \\
\mathcal{L}_{\xi q}^{(2)} & =\bar{\xi}_{n} \frac{\not h}{2}\left(g n \cdot A^{c}+i \not D_{\perp}^{c} \frac{1}{i \bar{n} \cdot D_{c}} g A_{\perp}^{c}\right) q_{u s}-\bar{\xi}_{n} i D_{\perp}^{u s} \frac{1}{i \bar{n} \cdot D_{c}} g \bar{n} \cdot A^{c} q_{u s}+\text { h.c. },
\end{aligned}
$$

where the superscripts denote the power suppression in $\lambda$ and $\lambda^{2}$. In each of the last terms in $\mathcal{L}_{\xi q}^{(1,2)}$ we use $1-W=1 /\left(i \bar{n} \cdot D_{c}\right) g \bar{n} \cdot A_{c}$, and in the first term of $\mathcal{L}_{\xi q}^{(2)}$ we write $g A_{\perp}^{c}=i \not D_{\perp}-P^{\perp}$ and then $q_{u s}=[W+(1-W)] q_{u s}$. Thus,

$$
\begin{aligned}
\mathcal{L}_{\xi q}^{(1)}= & \bar{\xi}_{n}\left(i \not D_{\perp}^{c} W-\mathbb{P}_{\perp}\right) q_{u s}+\text { h.c. }, \\
\mathcal{L}_{\xi q}^{(2)}= & \bar{\xi}_{n} \frac{\not h}{2}\left(g n \cdot A^{c}+i \not D_{\perp}^{c} \frac{1}{i \bar{n} \cdot D_{c}}\left(i \not D_{\perp}^{c}-\mathcal{P}_{\perp}\right)\right)[W+(1-W)] q_{u s}+\bar{\xi}_{n} i \not D_{\perp}^{u s}(W-1) q_{u s}+\text { h.c. } \\
= & \bar{\xi}_{n} \frac{\not h}{2}\left(g n \cdot A^{c}+i \not D_{\perp}^{c} \frac{1}{i \bar{n} \cdot D_{c}} i \not D_{\perp}^{c}\right) W q_{u s}-\bar{\xi}_{n} \frac{\not h}{2} i \not D_{\perp}^{c} \frac{1}{i \bar{n} \cdot D_{c}} \mathcal{P}_{\perp} q_{u s} \\
& +\bar{\xi}_{n}\left(\frac{\not h}{2} i n \cdot D^{u s}+i \not \not_{\perp}^{u s}\right)(W-1) q_{u s}+\text { h.c. }
\end{aligned}
$$

In manipulating $\mathcal{L}_{\xi q}^{(2)}$ we used the fact that integration by parts is allowed on the $(1-W) q_{u s}$ term and we can then use the equation of motion for the collinear quark to give a term $\left(-i n \cdot D_{u s}\right)(1-W)$ which we collected with the $i \not D_{\perp}^{u s}(W-1)$ term. The result in Eq. (30) agrees with Ref. [13], up to the fact that we performed the multipole expansion in momentum space.

In Eq. (30) we did not drop the $\mathscr{P}_{\perp} q=0$ terms since we want to make explicit the fact that it is the combination $\left(i \not D_{\perp} W-\mathcal{P}_{\perp}\right)=\left[i \not D_{\perp} W\right]$ which starts with at least one-collinear gluon. Written this way it appears that our $\mathcal{L}_{\xi q}^{(1)}$ is not collinear gauge invariant. In the transformed result the non-invariant term cancels if we use $\mathcal{P}_{\perp} q_{u s}=0$, but then it is not explicit that the operator starts with one-collinear gluon, so $\mathcal{L}_{\xi q}^{(1)}$ has either one or the other explicit. For $\mathcal{L}_{\xi q}^{(2)}$ Eq. (30) still involves the gluon field $A_{n}^{\mu}$ so the gauge invariance of this expression is not at all clear. However, the above considerations indicates that it should be possible to write all the terms in Eq. (30) in terms of gluon field strengths, and thereby achieve a manifestly gauge invariant action that starts with one-collinear gluon. This derivation was carried out in Ref. [15], but no details of the calculation were described there. These details are described below in Eqs. (31) through (35).

To proceed we note that using Eq. (11), ig $B_{c \perp}^{\nu} W=\left[i \bar{n} \cdot D_{c}, i D_{c}^{\perp \nu}\right] W=i \bar{n} \cdot D_{c} i D_{c}^{\perp \nu} W-$ $i D_{c}^{\perp \nu} W \overline{\mathcal{P}}$. Making similar manipulations for $n \cdot M$ and $M_{\perp}$ we can write

$$
\begin{aligned}
i g \not P_{c}^{\perp} W & =i \bar{n} \cdot D_{c}\left(i \not D_{c}^{\perp} W-\mathcal{P}_{\perp}\right)-\left\{i \not D_{c}^{\perp} W-\mathcal{P}_{c}^{\perp}\right\} \overline{\mathcal{P}}+\left\{g \bar{n} \cdot A^{c}\right\} \mathcal{P}_{\perp} . \\
i g n \cdot M W & =i \bar{n} \cdot D_{c} i n \cdot D W-\overline{\mathcal{P}} i n \cdot D_{u s}-\left\{i n \cdot D W-i n \cdot D_{u s}\right\} \overline{\mathcal{P}}, \\
i g M_{\perp} W & =i \bar{n} \cdot D_{c} i \not D_{\perp}^{u s} W-\overline{\mathcal{P}} i \not D_{\perp}^{u s}+\left\{i \not D_{\perp}^{u s}-i \not D_{\perp}^{u s} W\right\} \overline{\mathcal{P}} .
\end{aligned}
$$


Now we take purely usoft fields on the right, and divide on the left by $i \bar{n} \cdot D_{c}$. In Eq. (31) the terms in curly brackets start at one-collinear gluon, so even in the presence of $1 /\left(i \bar{n} \cdot D_{c}\right)$ these terms are non-singular and can safely be dropped using that the label operators give zero on the usoft field. This gives

$$
\begin{aligned}
\frac{1}{i \bar{n} \cdot D_{c}} i g \not B^{\perp} W q_{u s} & =\left(i \not D_{c}^{\perp} W-\not{P}^{\perp}\right) q_{u s}, \\
\frac{1}{i \bar{n} \cdot D_{c}} i g n \cdot M W q_{u s} & =\left(i n \cdot D W-i n \cdot D_{u s}\right) q_{u s}-(W-1) i n \cdot D_{u s} q_{u s}, \\
\frac{1}{i \bar{n} \cdot D_{c}} i g M M_{\perp} W q_{u s} & =i \not D_{\perp}^{u s}(W-1) q_{u s}-(W-1) i \not D_{\perp}^{u s} q_{u s} .
\end{aligned}
$$

These expressions allow us to write covariant derivatives acting on Wilson lines in terms of field strengths.

Using Eq. (32) for $\mathcal{L}_{\xi q}^{(1)}$ in Eq. (30), we arrive at the final result

$$
\mathcal{L}_{\xi q}^{(1)}=\bar{\xi}_{n} \frac{1}{i \bar{n} \cdot D_{c}} i g \not_{c}^{\perp} W q_{u s}+\text { h.c. } .
$$

This form is particularly nice since it is explicitly collinear and usoft gauge invariant and furthermore explicitly starts at one-collinear gluon due to the $B_{\perp}$. To see the gauge invariance note that under a collinear gauge transformation $U_{c}$ we have $\xi_{n} \rightarrow U_{c} \xi_{n}, W \rightarrow U_{c} W$, $\not B_{\perp} \rightarrow U_{c} \mathbb{B}_{\perp} U_{c}^{\dagger}$, and $\left(\bar{n} \cdot D_{c}\right)^{-1} \rightarrow U_{c}\left(\bar{n} \cdot D_{c}\right)^{-1} U_{c}^{\dagger}$ so all factors of $U_{c}$ cancel. Under an ultrasoft gauge transformation $U_{u}$ we have $\xi_{n} \rightarrow U_{u} \xi_{n}, W \rightarrow U_{u} W U_{u}^{\dagger}, \mathbb{B}_{\perp} \rightarrow U_{u} \mathbb{B}_{\perp} U_{u}^{\dagger}$, $\left(\bar{n} \cdot D_{c}\right)^{-1} \rightarrow U_{u}\left(\bar{n} \cdot D_{c}\right)^{-1} U_{u}^{\dagger}$, and $q_{u s} \rightarrow U_{u} q_{u s}$ so all factors of $U_{u}$ also cancel. In Fig. 3 in section $\mathrm{VC}$ we show the one and two gluon Feynman rules that follow from $\mathcal{L}_{u c}^{(1)}$ in Eq. (33). A non-trivial check on our manipulations is that the same Feynman rules can be obtained from Eq. (30) by using the free equations of motion.

We now proceed to further simplify $\mathcal{L}_{\xi q}^{(2)}$ in Eq. (30). Using Eq. (32) leaves

$$
\begin{aligned}
\mathcal{L}_{\xi q}^{(2)}= & \bar{\xi}_{n} \frac{1}{i \bar{n} \cdot D_{c}} i g M W q_{u s}+\bar{\xi}_{n} \frac{\not h}{2} i \not D_{\perp}^{c} \frac{1}{\left(i \bar{n} \cdot D_{c}\right)^{2}} i g B_{\perp}^{c} W q_{u s} \\
& +\bar{\xi}_{n}(W-1) i \not D_{u s} q_{u s}+\text { h.c. },
\end{aligned}
$$

where in the first and last terms we used the fact that $\not \xi_{n}=0$ to write a full $\gamma^{\mu}$ in $M$ and $\not D_{u s}$. For the last term in Eq. (34) we can now use the equation of motion for the usoft quark field to give our final result

$$
\mathcal{L}_{\xi q}^{(2)}=\bar{\xi}_{n} \frac{1}{i \bar{n} \cdot D_{c}} i g M W q_{u s}+\bar{\xi}_{n} \frac{\not h}{2} i \not D_{\perp}^{c} \frac{1}{\left(i \bar{n} \cdot D_{c}\right)^{2}} i g B_{\perp}^{c} W q_{u s}+\text { h.c. }
$$

Again in this form the action is explicitly collinear and usoft gauge invariant and furthermore explicitly starts at one-collinear gluon due to the field strength $B$ 's and $M$ 's. In the way we have written the result it is invariant under usoft Lorentz transformations on $x^{\mu}$ which separately rotate $\gamma_{\mu}$ and $D_{u s}^{\mu}$.

Finally we note that the mixed usoft-collinear quark actions in Eqs. (33) and (35) proved to be important for the proof of a factorization formula for heavy-to-light decays in Ref. 15]. In the next section we analyze the most general possible basis for $\mathcal{L}_{\xi q}^{(1,2)}$ beyond tree level, which follow purely from symmetry considerations and also discuss power suppressed terms in the collinear gluon action. 


\section{B. Most General Basis for $\mathcal{L}_{u c}$}

The ultrasoft-collinear quark Lagrangian can be expanded in a power series in the parameter $\lambda$. It is not possible to construct an invariant operator that is dimension- 4 and order $\lambda^{0}$. Therefore, we have the series

$$
\mathcal{L}_{\xi q}=\mathcal{L}_{\xi q}^{(1)}+\mathcal{L}_{\xi q}^{(2)}+\ldots
$$

Since this is a Lagrangian, insertions of these operators do not inject momentum, and we are free to integrate by parts as long as we are careful not to generate singular terms.

To construct the most general quark action $\mathcal{L}_{\xi q}^{(1)}$ we can use a single collinear quark field $\xi_{n} \sim \lambda$, an ultrasoft quark field $q_{u s} \sim \lambda^{3}$, and a $D_{c}^{\perp \mu} \sim \lambda$. These factors give a dimension 4 operator, and from the power counting formula in Eq. (10) they give $\delta=1$ which is the correct order for $\mathcal{L}_{\xi q}^{(1)}$. To satisfy collinear gauge invariance without changing the order in the power counting we make use of the Wilson line $W$ to write $\left(\bar{\xi}_{n} W\right)$ and $\left(W^{\dagger} i D_{c}^{\perp \mu} W\right)$. Since the Lagrangian is a scalar we must dot the index $\mu$ into another vector. The possible Dirac structures are restricted by the fact that $\not h \xi_{n}=0$. They are also restricted by type-III RPI, for instance $\not h \gamma_{\mu}$ is not invariant and therefore is ruled out (in the case of the heavy-to-light currents we can make use of the product $n \cdot v$, so $n \cdot v \not h$ is allowed). Taking these constraints into account leaves $\gamma_{\mu}$ as the only possibility. Thus, we have reduced $\mathcal{L}_{u c}^{(1)}$ to the form

$$
\left(\bar{\xi}_{n} W\right) \rho(\overline{\mathcal{P}}, \mu)\left(W^{\dagger} i \not D_{c}^{\perp} W\right) q_{u s}+\text { h.c. },
$$

where the coefficient $\rho$ is dimensionless and $i \not D_{c}^{\perp}$ acts to the left or right. However, by typeIII RPI invariance the coefficient $\rho(\overline{\mathcal{P}}, \mu)$ can not be a function of $\overline{\mathcal{P}}$, leaving only $\rho(\mu)$. Now $\rho$ is a dimensionless function of the dimension-full parameter $\mu$ and can only be equal to a constant (assuming no new dynamical scales like $\Lambda_{\mathrm{QCD}}$ are generated by renormalizing $\left.\mathcal{L}_{u c}^{(1)}\right)$. Now $\mathcal{P}_{\perp} q=0$ since $q$ carries no perpendicular momenta of order $\lambda$, so using Eq. (14) we see that $\left(W^{\dagger} i \overrightarrow{D D}_{c}^{\perp} W\right)$ can be traded for a $\not B_{c}^{\perp}$ operator. For $i \overleftarrow{\not D} \perp$ we obtain the same $\not B_{c}^{\perp}$ operator, plus $\left(\bar{\xi}_{n} W\right) \mathcal{P}_{\perp}^{\dagger} q=0$, which follows from the fact that $q_{u s}$, and by momentum conservation $\left(\bar{\xi}_{n} W\right)$, carries zero collinear $\perp$ momentum. Fixing the constant $\rho=1$ by tree level matching then leaves

$$
\mathcal{L}_{\xi q}^{(1)}=\bar{\xi}_{n} W \frac{1}{\overline{\mathcal{P}}} W^{\dagger} i g \mathbb{B}_{c}^{\perp} W q_{u s}+\text { h.c. }=\bar{\xi}_{n} \frac{1}{i \bar{n} \cdot D_{c}} i g \not B_{c}^{\perp} W q_{u s}+\text { h.c. . }
$$

In this form it is clear that the operator is collinear and usoft gauge invariant and generates terms with $\geq 1$ collinear gluon as required by momentum conservation. Finally, it is easy to show that its order $\lambda^{0}$ type-I and type-II variations vanish, since from Eq. (21) only the transformation of $D_{c}^{\perp}$ must be considered and $\delta_{I}^{\left(\lambda^{0}\right)} B_{c}^{\perp} \propto\left[i \bar{n} \cdot D_{c}, i \bar{n} \cdot D_{c}\right]=0$, while $\delta_{\mathrm{II}}^{\left(\lambda^{0}\right)} \not B_{c}^{\perp} \propto \not h$ which gives zero since $\not \xi_{n}=0$.

The above line of reasoning can be repeated at $\mathcal{O}\left(\lambda^{2}\right)$. By power counting and gauge invariance we can now have one $\left(W^{\dagger} i n \cdot D W\right)$ or two $\left(W^{\dagger} D_{c}^{\perp \mu} W\right)$ factors with derivatives to the left or right. Again we can use Eq. (14) to simplify the covariant derivative terms. We must have an operator starting with one-collinear gluon, and again the $\left(W^{\dagger} D W\right)$ factor next to $q_{u s}$ must be in square brackets and can be turned into a gluon field strength. Also 
by type-III RPI the Wilson coefficients must again be numbers, except for operators with three or more invariant collinear products in $\mathcal{L}_{\xi q}^{(2)}$ where they can be functions of the ratio $z_{i}$ of minus-momenta. Taking into account these constraints leaves three possible operators ${ }^{7}$

$$
\begin{aligned}
\mathcal{L}_{1}^{(2)} & =\rho_{1}\left(\bar{\xi}_{n} W\right)\left(W^{\dagger} \frac{1}{i \bar{n} \cdot D_{c}} i g M W\right) q_{u s}+\text { h.c. } \\
\mathcal{L}_{2}^{(2)} & =\int d z_{1} d z_{2} \rho_{2}\left(\frac{z_{2}}{z_{1}}\right)\left(\bar{\xi}_{n} W\right)_{z_{1}} \frac{\not h}{2}\left(W^{\dagger} i \vec{D}_{c}^{\perp} W\right)_{z_{2}} \frac{1}{\overline{\mathcal{P}}}\left(W^{\dagger} \frac{1}{i \bar{n} \cdot D_{c}} i g \mathbb{B}_{c}^{\perp} W\right) q_{u s}+\text { h.c. } \\
\mathcal{L}_{3}^{(2)} & =\int d z_{1} d z_{2} \rho_{3}\left(\frac{z_{2}}{z_{1}}\right)\left(\bar{\xi}_{n} W\right)_{z_{1}} \frac{\not h}{2}\left(W^{\dagger} i \vec{D}_{c}^{\perp \mu} W\right)_{z_{2}} \frac{1}{\overline{\mathcal{P}}}\left(W^{\dagger} \frac{1}{i \bar{n} \cdot D_{c}} i g B_{c \mu}^{\perp} W\right) q_{u s}+\text { h.c. },
\end{aligned}
$$

where $(\ldots)_{z_{i}} \equiv\left[\ldots \delta\left(z_{i}-\overline{\mathcal{P}}^{\dagger}\right)\right]$, and we've used our freedom to integrate by parts to make the perp covariant derivatives act to the right. Note that the overall $\bar{n} \cdot p$ momentum is zero, so no $z_{i}$ label is used on the $\not_{c}^{\perp}$ bracketed term (following the convention in Ref. [3] ). Again the presence or absence of factors of $\not h$ are completely fixed by type-III RPI. Now consider the type-I and type-II RPI transformations. Computing the order $\lambda$ variations of $\mathcal{L}_{u c}^{(1)}$ and simplifying the resulting expressions gives

$$
\begin{aligned}
\delta_{\mathrm{I}}^{(\lambda)} \mathcal{L}_{\xi q}^{(1)} & =-\bar{\xi}_{n} \frac{\not h}{2} \frac{1}{i \bar{n} \cdot D_{c}} i g \not \not_{\perp}^{c} \frac{\phi_{\perp}}{2} W q_{u s}-\bar{\xi}_{n} \frac{1}{i \bar{n} \cdot D_{c}}\left(\delta_{\mathrm{I}}^{\left(\lambda^{0}\right)} i g M_{\perp}\right) W q_{u s}+\text { h.c. }, \\
\delta_{\mathrm{II}}^{(\lambda)} \mathcal{L}_{\xi q}^{(1)} & =-\bar{\xi}_{n}\left\{i \not D_{c}^{\perp} \frac{1}{\left(i \bar{n} \cdot D_{c}\right)^{2}} i g \not \not_{c}^{\perp} \frac{\xi_{\perp}}{2}+\frac{1}{i \bar{n} \cdot D_{c}} \frac{\xi_{\perp}}{2} i g n \cdot M+\frac{1}{i \bar{n} \cdot D_{c}}\left(\delta_{\mathrm{II}}^{\left(\lambda^{0}\right)} i g M_{\perp}\right)\right\} W q_{u s}+\text { h.c. }
\end{aligned}
$$

The $\delta_{I, I I}^{\left(\lambda^{0}\right)} i g M_{\perp}$ terms appear since in the RPI transformations in Eq. (22) it is the full $D_{\perp}^{\mu}$ which transforms. For the order $\lambda^{0}$ variations of $\mathcal{L}_{j}^{(2)}$ we find

$$
\begin{aligned}
& \delta_{\mathrm{I}}^{\left(\lambda^{0}\right)} \mathcal{L}_{1}^{(2)}=\rho_{1} \bar{\xi}_{n}\left\{\frac{1}{i \bar{n} \cdot D_{c}} \frac{\not h}{2} \Delta_{\perp} \cdot B_{\perp}+\frac{1}{i \bar{n} \cdot D_{c}}\left(\delta_{\mathrm{I}}^{\left(\lambda^{0}\right)} i g M_{\perp}\right)\right\} W q_{u s}+\text { h.c. }, \\
& \delta_{\mathrm{II}}^{\left(\lambda^{0}\right)} \mathcal{L}_{1}^{(2)}=\rho_{1} \bar{\xi}_{n}\left\{\frac{1}{i \bar{n} \cdot D_{c}} \frac{\xi_{\perp}}{2} i g n \cdot M+\frac{1}{i \bar{n} \cdot D_{c}}\left(\delta_{\mathrm{II}}^{\left(\lambda^{0}\right)} i g M_{\perp}\right)\right\} W q_{u s}+\text { h.c. }, \\
& \delta_{\mathrm{I}}^{\left(\lambda^{0}\right)} \mathcal{L}_{2}^{(2)}=-\int d z_{1} d z_{2} \rho_{2}\left(\frac{z_{2}}{z_{1}}\right)\left(\bar{\xi}_{n} W\right)_{z_{1}} \delta\left(z_{2}\right) \frac{\not h \phi_{\perp}}{4} \frac{1}{\overline{\mathcal{P}}}\left(W^{\dagger} \frac{1}{i \bar{n} \cdot D_{c}} i g \mathbb{B}_{c}^{\perp} W\right) q_{u s}+\text { h.c. }, \\
& \delta_{\mathrm{II}}^{\left(\lambda^{0}\right)} \mathcal{L}_{2}^{(2)}=-\int d z_{1} d z_{2} \rho_{2}\left(\frac{z_{2}}{z_{1}}\right)\left(\bar{\xi}_{n} W\right)_{z_{1}}\left(W^{\dagger} i \overrightarrow{D D}_{c}^{\perp} W\right)_{z_{2}} \frac{\notin_{\perp}}{2} \frac{1}{\overline{\mathcal{P}}}\left(W^{\dagger} \frac{1}{i \bar{n} \cdot D_{c}} i g \mathbb{B}_{c}^{\perp} W\right) q_{u s} \\
& +\int d z_{1} d z_{2} \rho_{2}\left(\frac{z_{2}}{z_{1}}\right)\left(\bar{\xi}_{n} W\right)_{z_{1}}\left(W^{\dagger} i \vec{D}_{c}^{\perp} W\right)_{z_{2}} \frac{1}{\overline{\mathcal{P}}}\left(W^{\dagger} \frac{1}{i \bar{n} \cdot D_{c}} i g \varepsilon_{\perp} \cdot B_{c}^{\perp} W\right) q_{u s}+\text { h.c. }, \\
& \delta_{\mathrm{I}}^{\left(\lambda^{0}\right)} \mathcal{L}_{3}^{(2)}=-\int d z_{1} d z_{2} \rho_{3}\left(\frac{z_{2}}{z_{1}}\right)\left(\bar{\xi}_{n} W\right)_{z_{1}} \delta\left(z_{2}\right) \frac{\not h}{4} \frac{1}{\overline{\mathcal{P}}}\left(W^{\dagger} \frac{1}{i \bar{n} \cdot D_{c}} i g \Delta^{\perp} \cdot B_{c}^{\perp} W\right) q_{u s}+\text { h.c. }, \\
& \delta_{\mathrm{II}}^{\left(\lambda^{0}\right)} \mathcal{L}_{3}^{(2)}=\int d z_{1} d z_{2} \rho_{3}\left(\frac{z_{2}}{z_{1}}\right)\left(\bar{\xi}_{n} W\right)_{z_{1}} \frac{\notin_{\perp}}{2}\left(W^{\dagger} i \vec{D}_{c}^{\perp \mu} W\right)_{z_{2}} \frac{1}{\overline{\mathcal{P}}}\left(W^{\dagger} \frac{1}{i \bar{n} \cdot D_{c}} i g B_{c \mu}^{\perp} W\right) q_{u s} .
\end{aligned}
$$

\footnotetext{
7 This assumes we have eliminated a possible four quark operator using the collinear gluon equations of motion [15], $g^{2}\left(\bar{\xi}_{n} W T^{A} \not \vec{h} W^{\dagger} \xi_{n}\right) 1 / \overline{\mathcal{P}}^{2}\left(\bar{\xi}_{n} W T^{A} \not \vec{h} q_{u s}\right)=\bar{\xi}_{n} \not \vec{h} /\left(2 i \bar{n} \cdot D_{c}\right) i g n \cdot M W q_{u s}+2 \bar{\xi}_{n} \not \vec{n} /\{2(i \bar{n}$. $\left.\left.D_{c}\right)^{2}\right\}\left[i D_{c \mu}^{\perp}, i g B_{c}^{\perp \mu}\right] W q_{u s}$. This conclusion is not changed if we consider the most general possible fourquark operators allowed by all the symmetry constraints.
} 
Comparing Eqs. (40) and (41) we see that it is not possible to form an invariant involving $\mathcal{L}_{3}^{(2)}$ so $\rho_{3}=0$, while an invariant can be formed from $\mathcal{L}_{u c}^{(1)}+\mathcal{L}_{1}^{(2)}+\mathcal{L}_{2}^{(2)}$ by taking $\rho_{1}=1$ and $\rho_{2}\left(z_{2} / z_{1}\right)=1$. Since $\rho_{2}$ is independent of $z_{1} / z_{2}$ the integrals over $z_{1,2}$ can be performed. Therefore, we can write our final result for the first two orders in the usoft-collinear quark Lagrangian as

$$
\begin{aligned}
\mathcal{L}_{\xi q}^{(1)} & =\bar{\xi}_{n} \frac{1}{i \bar{n} \cdot D_{c}} i g \not_{c}^{\perp} W q_{u s}+\text { h.c. }, \\
\mathcal{L}_{\xi q}^{(2 a)} & =\bar{\xi}_{n} \frac{1}{i \bar{n} \cdot D_{c}} i g M W q_{u s}+\text { h.c. }, \\
\mathcal{L}_{\xi q}^{(2 b)} & =\bar{\xi}_{n} \frac{\not h}{2} i D_{\perp}^{c} \frac{1}{\left(i \bar{n} \cdot D_{c}\right)^{2}} i g \mathbb{B}_{\perp}^{c} W q_{u s}+\text { h.c. }
\end{aligned}
$$

These terms agree exactly with the result from tree level matching in Eqs. (33) and (35). The analysis here shows that no other terms are induced by matching at any order in $\alpha_{s}$.

Next we proceed to analyze power suppressed terms in the collinear gluon action. Starting with the LO collinear gluon action [4], $\mathcal{L}_{c g}^{(0)}$ and making it RPI invariant with $i D^{\mu}=i D_{c}^{\mu}+$ $i D_{u s}^{\mu}$ gives

$$
\mathcal{L}_{c g}=\frac{1}{2 g^{2}} \operatorname{tr}\left\{\left[i D^{\mu}, i D^{\nu}\right]^{2}\right\}
$$

It is straightforward to see that no other gauge invariant pure glue dimension-4 operators are possible. We could build a more general gauge invariant operator out of a string of $m$ terms $\left[\mathcal{W} i D^{\mu_{i}} \mathcal{W}^{\dagger}\right]_{z_{i}}$, with $m-4$ factors of $1 / \overline{\mathcal{P}}$ to make up the mass dimensions. However, type-III RPI then demands $m-4$ factors of $\bar{n}_{\mu_{i}}$ in the numerators which using $\bar{n} \cdot D \mathcal{W}=0$ collapses the operator to the case $m=4$. Finally, since $\mathcal{W}$ transforms under type-II RPI as in Eq. (22), but $i D^{\mu}$ does not we find that these operators must have Wilson coefficients $C\left(z_{i}\right)$ that are independent of the $z_{i}$ parameters. In this case all factors of $\mathcal{W}$ cancel out and we are left with Eq. (43) (after performing the $z_{i}$ integrals and fixing the coefficient at tree level). Expanding Eq. (43) we see that the order $\lambda$ and $\lambda^{2}$ suppressed terms are

$$
\begin{aligned}
\mathcal{L}_{c g}^{(1)} & =\frac{2}{g^{2}} \operatorname{tr}\left\{\left[i \mathcal{D}^{\mu}, i D_{c}^{\perp \nu}\right]\left[i \mathcal{D}_{\mu}, i D_{u s \nu}^{\perp}\right]\right\}, \\
\mathcal{L}_{c g}^{(2)} & =\frac{1}{g^{2}} \operatorname{tr}\left\{\left[i \mathcal{D}^{\mu}, i D_{u s}^{\perp \nu}\right]\left[i \mathcal{D}_{\mu}, i D_{u s \nu}^{\perp}\right]\right\}+\frac{1}{g^{2}} \operatorname{tr}\left\{\left[i D_{u s}^{\perp \mu}, i D_{u s}^{\perp \nu}\right]\left[i D_{c \mu}^{\perp}, i D_{c \nu}^{\perp}\right]\right\} \\
& +\frac{1}{g^{2}} \operatorname{tr}\left\{\left[i \mathcal{D}^{\mu}, i n \cdot D\right]\left[i \mathcal{D}_{\mu}, i \bar{n} \cdot D_{u s}\right]\right\}+\frac{1}{g^{2}} \operatorname{tr}\left\{\left[i D_{u s}^{\perp \mu}, i D_{c}^{\perp \nu}\right]\left[i D_{c \mu}^{\perp}, i D_{u s \nu}^{\perp}\right]\right\},
\end{aligned}
$$

where $\mathcal{D}^{\mu}=D_{c}^{\mu}+\bar{n}^{\mu} n \cdot D_{u s} / 2$.

In Ref. [4] the gauge fixing terms in the LO gluon action were given in a general covariant gauge. We do not bother to consider the possibility of other leading order gauge fixing terms since we have some residual freedom to choose these terms however we like. In an RPI invariant form the terms from Ref. [4] are

$$
\mathcal{L}_{c g}=2 \operatorname{tr}\left\{\bar{c}_{n}\left[i D_{\mu},\left[i D^{\mu}, c_{n}\right]\right]\right\}+\frac{1}{\alpha} \operatorname{tr}\left\{\left[i D_{\mu}, A_{n}^{\mu}\right]\left[i D_{\nu}, A_{n}^{\nu}\right]\right\}
$$


and the subleading terms in their expansion are

$$
\begin{aligned}
\mathcal{L}_{c g}^{(1)}= & 2 \operatorname{tr}\left\{\bar{c}_{n}\left[i D_{\perp \mu}^{u s},\left[i D_{c}^{\perp \mu}, c_{n}\right]\right]\right\}+2 \operatorname{tr}\left\{\bar{c}_{n}\left[i D_{\perp \mu}^{c},\left[i D_{u s}^{\perp \mu}, c_{n}\right]\right]\right\} \\
& +\frac{2}{\alpha} \operatorname{tr}\left\{\left[i D_{\perp \mu}^{u s}, A_{n}^{\perp \mu}\right]\left[i D_{\nu}, A_{n}^{\nu}\right]\right\}, \\
\mathcal{L}_{c g}^{(2)}= & 2 \operatorname{tr}\left\{\bar{c}_{n}\left[i D_{\perp \mu}^{u s},\left[i D_{u s}^{\perp \mu}, c_{n}\right]\right]\right\}+\operatorname{tr}\left\{\bar{c}_{n}\left[i \bar{n} \cdot D^{u s},\left[i n \cdot D, c_{n}\right]\right]\right\} \\
& +\operatorname{tr}\left\{\bar{c}_{n}\left[i n \cdot D,\left[i \bar{n} \cdot D_{u s}, c_{n}\right]\right]\right\} \\
& +\frac{1}{\alpha} \operatorname{tr}\left\{\left[i D_{\perp \mu}^{u s}, A_{n}^{\perp \mu}\right]\left[i D_{\perp \nu}^{u s}, A_{n}^{\perp \nu}\right]\right\}+\frac{1}{\alpha} \operatorname{tr}\left\{\left[i \bar{n} \cdot D^{u s}, n \cdot A_{n}\right]\left[i D_{\nu}, A_{n}^{\nu}\right]\right\} .
\end{aligned}
$$

\section{MOST GENERAL BASIS FOR HEAVY-TO-LIGHT CURRENTS}

In this section we give our derivation of the most general basis of heavy-to-light currents at $\mathcal{O}(\lambda)$. The scalar current is given in great detail, and forms the basis of the analysis for the other Dirac structures. Expanding the heavy-to-light currents in powers of $\lambda$ we write

$$
J_{h l}=J^{(d)}+K^{(d)}+\ldots,
$$

for the LO currents $\left(J^{(d)}\right)$, and NLO currents $\left(K^{(d)}\right)$. The superscript denotes whether the current is scalar $(d=s)$, pseudo-scalar $(d=p)$, vector $(d=v)$, axial-vector $(d=a)$, or tensor $(d=t)$. For the preliminary basis where only constraints from gauge invariance, power counting, and type-III RPI invariance are imposed we use a calligraphic notation $\mathcal{J}^{(d)}+\mathcal{K}^{(d)}+\ldots$, and then switch to Roman for the final basis that is invariant under all the type-I and type-II constraints. We will also make use of a convolution notation

$$
J^{(d)}=\int d \omega C^{(d)}\left(\frac{\omega}{m_{b}}, \frac{\mu}{m_{b}}\right) J^{(d)}(\omega),
$$

where $J^{(d)}(\omega)$ contains fields and operators with the notation in Eq. (8) and the Wilson coefficients $C^{(d)}\left(\hat{\omega}, \mu / m_{b}\right)$ are numerical functions of the convolution parameter (where $\hat{\omega}=$ $\left.\omega / m_{b}\right)$.

\section{A. Scalar Currents}

From gauge invariance and power counting the most general leading order heavy-to-light current has the form $\bar{\xi}_{n} W \Gamma h_{v}$ [2]. For $v_{\perp} \neq 0$ the most general allowed scalar spin structures from section $I \mathrm{~B}$ are then $\Gamma=\{1, \not h\}$. Type-III RPI demands that the $\not{h}$ is accompanied by either a $n \cdot v$ or a $1 / \bar{n} \cdot v$. Thus after imposing constraints $\mathrm{i}$ )-iv) of section $\coprod$ we are left with the possible leading order currents

$$
\mathcal{J}_{1}^{(s)}=c_{1}^{(s)} \bar{\xi}_{n} W h_{v}, \quad \mathcal{J}_{2}^{(s)}=c_{2}^{(s)} \bar{\xi}_{n} W n \cdot v \frac{\not h}{2} h_{v}, \quad \mathcal{J}_{3}^{(s)}=c_{3}^{(s)} \bar{\xi}_{n} W \frac{\not h}{2 \bar{n} \cdot v} h_{v},
$$

where $c_{i}$ are dimensionless Wilson coefficients. With type-III RPI invariance the $c_{i}$ can only depend on the combination $(n \cdot v \overline{\mathcal{P}})$, the b-quark mass $m_{b}$, and the renormalization scale $\mu$. 
Now consider the order $\lambda^{0}$ type-I and II RPI transformations in Eq. (21). Since none of the operators in Eq. (50) involve quantities that have $\delta_{\mathrm{I}}^{\left(\lambda^{0}\right)}$ transformations they are invariant under type-I at this order. However, under the analogous type-II transformations

$$
\begin{aligned}
& \delta_{\mathrm{II}}^{\left(\lambda^{0}\right)} \mathcal{J}_{1}^{(s)}=0, \quad \delta_{\mathrm{II}}^{\left(\lambda^{0}\right)} \mathcal{J}_{2}^{(s)}=c_{2}^{(s)} \bar{\xi}_{n} W\left(n \cdot v \frac{\xi_{\perp}}{2}\right) h_{v}, \\
& \delta_{\mathrm{II}}^{\left(\lambda^{0}\right)} \mathcal{J}_{3}^{(s)}=c_{3}^{(s)} \bar{\xi}_{n} W\left(\frac{\notin_{\perp}}{2 \bar{n} \cdot v}-\frac{\not h}{2(\bar{n} \cdot v)^{2}} \varepsilon_{\perp} \cdot v\right) h_{v} .
\end{aligned}
$$

Thus, it is not possible to form an invariant involving the currents $\mathcal{J}_{2,3}^{(s)}$, and only the current $\mathcal{J}_{1}^{(s)}$ is allowed. Therefore, we can rewrite our final result for the most general leading order scalar current as

$$
J_{0}^{(s)}=C_{0}^{(s)}\left(\frac{-n \cdot v \overline{\mathcal{P}}}{m_{b}}, \frac{\mu}{m_{b}}\right) \bar{\xi}_{n} W h_{v} .
$$

Since the Wilson coefficient is dimensionless it can only be a function of the dimensionless ratios of parameters as shown. The minus sign in the first variable is included so that $\overline{\mathcal{P}}$ gives the total outgoing momentum of $\bar{\xi}_{n} W$. Switching to the convolution notation in Eq. (49) and defining $\hat{\omega}=\omega / m_{b}$ we can write Eq. (52) as

$$
\begin{aligned}
J_{0}^{(s)} & =\int d \omega C_{0}^{(s)}\left(\hat{\omega}, \mu / m_{b}\right) J_{0}^{(s)}(\omega), \\
J_{0}^{(s)}(\omega) & =\left(\bar{\xi}_{n} W\right)_{\omega} h_{v} .
\end{aligned}
$$

Thus our notation is that $J_{0}^{(s)}$ contains the Wilson coefficient, while $J_{0}^{(s)}(\omega)$ is purely the field operator. With the convolution notation in Eq. (153) the Wilson coefficients are just numerical functions which do not transform under RPI. We will often suppress the dependence of Wilson coefficients on $\mu / m_{b}$ in what follows.

Next consider currents that are suppressed by a power of $\lambda$. At this order the only additional structure we can use is a $D_{c}^{\perp \mu} \sim \lambda$, where the derivative acts to the left or to the right. To form the most general collinear gauge invariant we take $\left(W^{\dagger} D_{c}^{\perp \mu} W\right)$, which we then insert between the $\left(\bar{\xi}_{n} W\right)$ and $h_{v}$ to satisfy the usoft gauge invariance. Since the two collinear factors are invariant by themselves they can have arbitrary labels $\omega_{1,2}$. Thus we have operators with the structure

$$
\left(\bar{\xi}_{n} W\right)_{\omega_{1}} \Gamma\left(W^{\dagger} D_{c}^{\perp \mu} W\right)_{\omega_{2}} \frac{1}{\overline{\mathcal{P}}^{\dagger}} h_{v}
$$

The factor of $1 / \overline{\mathcal{P}}^{\dagger}$ is included to make the Wilson coefficients dimensionless. To make a scalar current the $\mu$ superscript in Eq. (54) can be dotted into a $\gamma_{\mu}$ or $v_{\mu}$ in $\Gamma$. In either case the most general remaining Dirac structure involves either 1 or $\not h$ as follows from section $\amalg B$. Thus, combining the constraints from gauge invariance, spin reduction, and type-III RPI leaves eight $\mathcal{O}(\lambda)$ suppressed currents

$$
\mathcal{K}_{j}^{(s)}=\int d \omega_{1} d \omega_{2} b_{j}^{(s)}\left(\hat{\omega}_{1}, \hat{\omega}_{2}\right) \mathcal{K}_{j}^{(s)}\left(\omega_{1}, \omega_{2}\right)
$$


where $j=1, \ldots, 8$, and the $b_{i}$ coefficients are dimensionless functions of $\hat{\omega}_{1,2}=\omega_{1,2} / m_{b}$ and $\mu / m_{b}$. The eight operators are

$$
\begin{aligned}
& \mathcal{K}_{\{1,2\}}^{(s)}\left(\omega_{1}, \omega_{2}\right)=\left(\bar{\xi}_{n} W\right)_{\omega_{1}}\left(W^{\dagger} i \overleftarrow{D}_{c}^{\perp} W\right)_{\omega_{2}} \frac{1}{\overline{\mathcal{P}}^{\dagger}}\left\{\frac{\not h}{2}, \frac{1}{n \cdot v}\right\} h_{v}, \\
& \mathcal{K}_{\{3,4\}}^{(s)}\left(\omega_{1}, \omega_{2}\right)=\left(\bar{\xi}_{n} W\right)_{\omega_{1}}\left(W^{\dagger} i v \cdot \overleftarrow{D}_{c}^{\perp} W\right)_{\omega_{2}} \frac{1}{\overline{\mathcal{P}}^{\dagger}}\left\{\frac{\not h}{2}, \frac{1}{n \cdot v}\right\} h_{v}, \\
& \mathcal{K}_{\{5,6\}}^{(s)}\left(\omega_{1}, \omega_{2}\right)=\left(\bar{\xi}_{n} W\right)_{\omega_{1}}\left(W^{\dagger} i \vec{D}_{c}^{\perp} W\right)_{\omega_{2}} \frac{1}{\overline{\mathcal{P}}^{\dagger}}\left\{\frac{\not h}{2}, \frac{1}{n \cdot v}\right\} h_{v}, \\
& \mathcal{K}_{\{7,8\}}^{(s)}\left(\omega_{1}, \omega_{2}\right)=\left(\bar{\xi}_{n} W\right)_{\omega_{1}}\left(W^{\dagger} i v \cdot \vec{D}_{c}^{\perp} W\right)_{\omega_{2}} \frac{1}{\overline{\mathcal{P}}^{\dagger}}\left\{\frac{\not h}{2}, \frac{1}{n \cdot v}\right\} h_{v}
\end{aligned}
$$

Note that the dependence of the Wilson coefficients on the labels $\omega_{i}$ account for insertions of $1 / \overline{\mathcal{P}}^{\dagger}$ in all possible locations. Just as for the leading currents we cannot use $\bar{n} \cdot v$ to form a type-III invariant in Eq. (56) as it leads to currents which can not be made invariant under type-II transformations (the transformed currents would depend on $\epsilon_{\perp} \cdot v /(\bar{n} \cdot v)^{2}$ in a way that could not be canceled).

Next consider the type-I transformations for the currents in Eq. (156). For these subleading currents only the $\lambda^{0}$ transformations are necessary since we are only working to order $\lambda$. Under type-I only $D_{c}^{\perp}$ transforms and we have

$$
\begin{aligned}
& \delta_{\mathrm{I}}^{\left(\lambda^{0}\right)}\left(W^{\dagger} i \overleftarrow{D}_{c}^{\perp \mu} W\right)_{\omega_{2}}=-\frac{\Delta_{\perp}^{\mu}}{2}\left(W^{\dagger} i \bar{n} \cdot \overleftarrow{D}_{c} W\right)_{\omega_{2}}=+\frac{\Delta_{\perp}^{\mu}}{2} \overline{\mathcal{P}}^{\dagger} \delta\left(\omega_{2}-n \cdot v \overline{\mathcal{P}}^{\dagger}\right) \\
& \delta_{\mathrm{I}}^{\left(\lambda^{0}\right)}\left(W^{\dagger} i \vec{D}_{c}^{\perp \mu} W\right)_{\omega_{2}}=-\frac{\Delta_{\perp}^{\mu}}{2}\left(W^{\dagger} i \bar{n} \cdot \vec{D}_{c} W\right)_{\omega_{2}}=-\frac{\Delta_{\perp}^{\mu}}{2} \overline{\mathcal{P}} \delta\left(\omega_{2}-n \cdot v \overline{\mathcal{P}}^{\dagger}\right)
\end{aligned}
$$

Since $\overline{\mathcal{P}} h_{v}=0$ it is easy to see that each of $\mathcal{K}_{5,6,7,8}^{(s)}$ are type-I invariant all by themselves. The other currents do transform, and using Eq. (57) gives

$$
\begin{aligned}
\delta_{\mathrm{I}}^{\left(\lambda^{0}\right)} \mathcal{K}_{1}^{(s)}\left(\omega_{1}, \omega_{2}\right) & =\delta\left(\omega_{2}\right)\left(\bar{\xi}_{n} W\right)_{\omega_{1}}\left(\frac{-\not h \Delta_{\perp}}{4}\right) h_{v}, \\
\delta_{\mathrm{I}}^{\left(\lambda^{0}\right)} \mathcal{K}_{2}^{(s)}\left(\omega_{1}, \omega_{2}\right) & =\delta\left(\omega_{2}\right)\left(\bar{\xi}_{n} W\right)_{\omega_{2}}\left(\frac{\Delta_{\perp}}{2 n \cdot v}\right) h_{v}, \\
\delta_{\mathrm{I}}^{\left(\lambda^{0}\right)} \mathcal{K}_{3}^{(s)}\left(\omega_{1}, \omega_{2}\right) & =\delta\left(\omega_{2}\right)\left(\bar{\xi}_{n} W\right)_{\omega_{1}}\left(\frac{\not h \Delta_{\perp} \cdot v}{4}\right) h_{v}, \\
\delta_{\mathrm{I}}^{\left(\lambda^{0}\right)} \mathcal{K}_{4}^{(s)}\left(\omega_{1}, \omega_{2}\right) & =\delta\left(\omega_{2}\right)\left(\bar{\xi}_{n} W\right)_{\omega_{1}}\left(\frac{\Delta_{\perp} \cdot v}{2 n \cdot v}\right) h_{v}, \\
\delta_{\mathrm{I}}^{\left(\lambda^{0}\right)} \mathcal{K}_{5,6,7,8}^{(s)}\left(\omega_{1}, \omega_{2}\right) & =0 .
\end{aligned}
$$

The delta functions $\delta\left(\omega_{2}\right)$ cause only the coefficients $b_{1,2,3,4}^{(s)}\left(\hat{\omega}_{1}, 0\right)$ to appear in the transformation of $\mathcal{K}_{\{1,2,3,4\}}^{(s)}$. We also need the order $\lambda$ variation of the LO current in Eq. (53). In this computation we must be careful to note that the $\delta\left(\omega-n \cdot v \overline{\mathcal{P}}^{\dagger}\right)$ in $\left(\bar{\xi}_{n} W\right)_{\omega}$ depends on $n \cdot v$, and therefore also transforms

$$
\delta_{I}^{\left(\lambda^{0}\right)} \delta\left(\omega-n \cdot v \overline{\mathcal{P}}^{\dagger}\right)=-v \cdot \Delta_{\perp} \overline{\mathcal{P}}^{\dagger} \delta^{\prime}\left(\omega-n \cdot v \overline{\mathcal{P}}^{\dagger}\right)=-\frac{v \cdot \Delta_{\perp}}{n \cdot v} \frac{d}{d \omega} \omega \delta\left(\omega-n \cdot v \overline{\mathcal{P}}^{\dagger}\right)
$$


Using Eq. (22) we find a term from transforming the delta function and a term from transforming the collinear quark field

$$
\delta_{\mathrm{I}}^{(\lambda)} J_{0}^{(s)}(\omega)=\left(\bar{\xi}_{n} W\right)_{\omega}\left(\frac{\not h \phi_{\perp}}{4}\right) h_{v}-\frac{v \cdot \Delta_{\perp}}{n \cdot v} \frac{d}{d \omega} \omega\left(\bar{\xi}_{n} W\right)_{\omega} h_{v} .
$$

Demanding invariance under the transformations in Eqs. (58) and (60) gives non-trivial constraints on the Wilson coefficients in $J_{0}^{(s)}$ and $\mathcal{K}_{i}^{(s)}$. From Eq. (58) the currents $\mathcal{K}_{\{1,2,3,4\}}^{(s)}$ are invariant by themselves provided that $b_{\{1,2,3,4\}}^{(s)}\left(\hat{\omega}_{1}, 0\right)=0$. However, in Eq. (60) the first term can only be canceled by a $\mathcal{K}_{1}^{(s)}$ with $b_{1}^{(s)}(\hat{\omega}, 0)=C_{0}^{(s)}(\hat{\omega})$. To cancel the second term we integrate by parts to give a $\omega d / d \omega=\hat{\omega} d / d \hat{\omega}$ acting on $C_{0}^{(s)}$. This term can then be canceled by $b_{4}^{(s)}\left(\hat{\omega}_{1}, 0\right)=-2 \hat{\omega} d / d \hat{\omega} C_{0}^{(s)}(\hat{\omega})$. Thus, the summary of type-I invariants is $J_{0}+\mathcal{K}_{1}^{(s)}+\mathcal{K}_{4}^{(s)}$, $\mathcal{K}_{\{5,6,7,8\}}^{(s)}, \mathcal{K}_{\{2,3\}}^{(s)}$, with any $b_{5,6,7,8}^{(s)}\left(\hat{\omega}_{1}, \hat{\omega}_{2}\right)$ and coefficients

$$
b_{1}^{(s)}(\hat{\omega}, 0)=C_{0}^{(s)}(\hat{\omega}), \quad b_{2,3}^{(s)}(\hat{\omega}, 0)=0, \quad b_{4}^{(s)}(\hat{\omega}, 0)=-2 \hat{\omega} d / d \hat{\omega} C_{0}^{(s)}(\hat{\omega}) .
$$

Now consider the type-II transformations. The analog of Eq. (59) is

$$
\delta_{\mathrm{II}}^{(\lambda)} \delta\left(\omega-n \cdot v \overline{\mathcal{P}}^{\dagger}\right)=-n \cdot v \varepsilon_{\perp} \cdot \mathcal{P}_{\perp}^{\dagger} \delta^{\prime}\left(\omega-n \cdot v \overline{\mathcal{P}}^{\dagger}\right)=-n \cdot v \varepsilon_{\perp} \cdot \mathcal{P}_{\perp}^{\dagger} \frac{d}{d \omega} \delta\left(\omega-n \cdot v \overline{\mathcal{P}}^{\dagger}\right)
$$

For the order $\lambda$ variation of the LO current $J_{0}^{(s)}(\omega)$ we have terms from the transformation of the delta function, the collinear quark field, and the Wilson line $W$

$$
\begin{aligned}
\delta_{\mathrm{II}}^{(\lambda)} J_{0}(\omega)= & n \cdot v \varepsilon_{\perp} \cdot \mathcal{P}_{\perp} \frac{d}{d \omega}\left(\bar{\xi}_{n} W\right)_{\omega} h_{v}+\left(\bar{\xi}_{n} i \overleftarrow{D D}_{c}^{\perp} \frac{1}{i \bar{n} \cdot \overleftarrow{D}_{c}} \frac{\xi_{\perp}}{2} W\right)_{\omega} h_{v} \\
& -\left(\bar{\xi}_{n} \frac{1}{i \bar{n} \cdot D_{c}} i \varepsilon_{\perp} \cdot \vec{D}_{c}^{\perp} W\right)_{\omega} h_{v} .
\end{aligned}
$$

In the subleading currents $\mathcal{K}_{i}^{(s)}$ both $\bar{n}^{\mu}$ and $D_{c}^{\perp}$ have $\lambda^{0}$ transformations. $^{8} \mathrm{~A} i \not D_{c}^{\perp}$ transforms to give a $\not h$, so since $\not h \xi_{n}=0$ it is easy to see that $\mathcal{K}_{\{2,6\}}^{(s)}$ are invariant under type-II transformations at this order. The transformations for the remaining currents are more involved

$$
\begin{aligned}
& \delta_{\mathrm{II}}^{\left(\lambda^{0}\right)} \mathcal{K}_{\{2,6\}}^{(s)}\left(\omega_{1}, \omega_{2}\right)=0, \\
& \delta_{\mathrm{II}}^{\left(\lambda^{0}\right)} \mathcal{K}_{1}^{(s)}\left(\omega_{1}, \omega_{2}\right)=\left(\bar{\xi}_{n} W\right)_{\omega_{1}}\left(W^{\dagger} i \overleftarrow{D D}_{c}^{\perp} W\right)_{\omega_{2}} \frac{\notin_{\perp}}{2} \frac{1}{\overline{\mathcal{P}}^{\dagger}} h_{v} \\
& \delta_{\mathrm{II}}^{\left(\lambda^{0}\right)} \mathcal{K}_{3}^{(s)}\left(\omega_{1}, \omega_{2}\right)=\left(\bar{\xi}_{n} W\right)_{\omega_{1}}\left\{\frac{\notin_{\perp}}{2}\left(W^{\dagger} i v \cdot \overleftarrow{D}_{c}^{\perp} W\right)_{\omega_{2}}-\frac{n \cdot v \not h}{4}\left(W^{\dagger} i \varepsilon_{\perp} \cdot \overleftarrow{D}{ }_{c}^{\perp} W\right)_{\omega_{2}}\right\} \frac{1}{\overline{\mathcal{P}}^{\dagger}} h_{v} \\
& \delta_{\mathrm{II}}^{\left(\lambda^{0}\right)} \mathcal{K}_{4}^{(s)}\left(\omega_{1}, \omega_{2}\right)=-\frac{1}{2}\left(\bar{\xi}_{n} W\right)_{\omega_{1}}\left(W^{\dagger} i \varepsilon_{\perp} \cdot \overleftarrow{D}_{c}^{\perp} W\right)_{\omega_{2}} \frac{1}{\overline{\mathcal{P}}^{\dagger}} h_{v} \\
& \delta_{\mathrm{II}}^{\left(\lambda^{0}\right)} \mathcal{K}_{5}^{(s)}\left(\omega_{1}, \omega_{2}\right)=\left(\bar{\xi}_{n} W\right)_{\omega_{1}}\left(W^{\dagger} i \overrightarrow{D D}_{c}^{\perp} W\right)_{\omega_{2}} \frac{\notin_{\perp}}{2} \frac{1}{\overline{\mathcal{P}}^{\dagger}} h_{v} \\
& \delta_{\mathrm{II}}^{\left(\lambda^{0}\right)} \mathcal{K}_{7}^{(s)}\left(\omega_{1}, \omega_{2}\right)=\left(\bar{\xi}_{n} W\right)_{\omega_{1}}\left\{\frac{\notin_{\perp}}{2}\left(W^{\dagger} i v \cdot \vec{D}_{c}^{\perp} W\right)_{\omega_{2}}-\frac{n \cdot v \not h}{4}\left(W^{\dagger} i \varepsilon_{\perp} \cdot \vec{D}_{c}^{\perp} W\right)_{\omega_{2}}\right\} \frac{1}{\overline{\mathcal{P}}^{\dagger}} h_{v}, \\
& \delta_{\mathrm{II}}^{\left(\lambda^{0}\right)} \mathcal{K}_{8}^{(s)}\left(\omega_{1}, \omega_{2}\right)=-\frac{1}{2}\left(\bar{\xi}_{n} W\right)_{\omega_{1}}\left(W^{\dagger} i \varepsilon_{\perp} \cdot \vec{D}_{c}^{\perp} W\right)_{\omega_{2}} \frac{1}{\overline{\mathcal{P}}^{\dagger}} h_{v},
\end{aligned}
$$

\footnotetext{
${ }^{8}$ Since $\delta_{\mathrm{II}} \overline{\mathcal{P}}=\mathcal{O}(\lambda)$ the transformation of the delta functions in $\mathcal{K}_{i}^{(s)}$ only appear at one higher order.
} 
It is straightforward to see that it is not possible to form a type-II invariant using only the currents $\mathcal{K}_{\{1,3,4,5,7,8\}}^{(s)}$. However, it is possible to form an invariant taking a combination of $\mathcal{K}_{\{1,4,8\}}^{(s)}$ with $J_{0}^{(s)}$. To facilitate this we rewrite Eq. (63) as

$$
\begin{gathered}
\delta_{\mathrm{II}}^{\left(\lambda^{1}\right)} J_{0}^{(s)}(\omega)=\int d \omega_{1}\left[n \cdot v \frac{d}{d \omega}\left(\bar{\xi}_{n} W\right)_{\omega_{1}}\left\{\left(W^{\dagger} i \varepsilon_{\perp} \cdot \vec{D}_{c}^{\perp} W\right)_{\omega-\omega_{1}}+\left(W^{\dagger} i \varepsilon_{\perp} \cdot \overleftarrow{D} \overleftarrow{c}_{c}^{\perp} W\right)_{\omega-\omega_{1}}\right\} h_{v}\right. \\
\left.-\left(\bar{\xi}_{n} W\right)_{\omega_{1}}\left(W^{\dagger} i \overleftarrow{D}_{c}^{\perp} W\right)_{\omega-\omega_{1}} \frac{1}{\overline{\mathcal{P}}^{\dagger}} \frac{\xi_{\perp}}{2} h_{v}-\left(\bar{\xi}_{n} W\right)_{\omega_{1}} \frac{1}{\omega_{1}-\omega}\left(W^{\dagger} i \varepsilon_{\perp} \cdot \vec{D}_{c}^{\perp} W\right)_{\omega-\omega_{1}} h_{v}\right] .
\end{gathered}
$$

To derive Eq. (65) we used $\varepsilon_{\perp} \cdot \mathcal{P}_{\perp} \bar{\xi}_{n} W h_{v}=\bar{\xi}_{n} i \varepsilon_{\perp} \cdot \vec{D}_{c}^{\perp} W h_{v}+\bar{\xi}_{n} i \varepsilon_{\perp} \cdot \overleftarrow{D}_{c}^{\perp} W h_{v}$, and the fact that the $\omega_{1}$ integration can be carried out with the delta function in $\left(\bar{\xi}_{n} W\right)_{\omega_{1}}$ to get back a product of operators with momentum label $\omega$ where the intermediate $W W^{\dagger}$ cancel out. Now for the $d / d \omega$ terms in Eq. (65) we can integrate by parts in $\left[C_{0}(\hat{\omega}) \delta_{\mathrm{II}}^{\left(\lambda^{1}\right)} J_{0}^{(s)}(\omega)\right]$ so that the derivative acts on the Wilson coefficient $C_{0}^{(s)}(\hat{\omega})$. It is then evident that the third term in Eq. (65) can be canceled by $\delta_{\mathrm{II}}^{\left(\lambda^{0}\right)} \mathcal{K}_{1}^{(s)}$ with $b_{1}^{(s)}\left(\hat{\omega}_{1}, \hat{\omega}-\hat{\omega}_{1}\right)=C_{0}^{(s)}(\hat{\omega})$, the second term is canceled by $\delta_{\mathrm{II}}^{\left(\lambda^{0}\right)} \mathcal{K}_{4}^{(s)}$ with $b_{4}^{(s)}\left(\hat{\omega}_{1}, \hat{\omega}-\hat{\omega}_{1}\right)=-2 \hat{\omega} d / d \hat{\omega} C_{0}^{(s)}(\hat{\omega})$, and the first and fourth terms are canceled by $\delta_{\mathrm{II}}^{\left(\lambda^{0}\right)} \mathcal{K}_{8}^{(s)}$ with $b_{8}^{(s)}\left(\hat{\omega}_{1}, \hat{\omega}-\hat{\omega}_{1}\right)=-2 \hat{\omega} d / d \hat{\omega} C_{0}^{(s)}(\hat{\omega})-2 \hat{\omega} /\left(\hat{\omega}_{1}-\hat{\omega}\right) C_{0}^{(s)}(\hat{\omega})$. Therefore, type-II RPI rules out the operators $\mathcal{K}_{3,5,7}^{(s)}$ and leaves only the invariants

$$
J_{0}^{(s)}+\mathcal{K}_{1}^{(s)}+\mathcal{K}_{4}^{(s)}+\mathcal{K}_{8}^{(s)}, \quad \mathcal{K}_{2}^{(s)}, \quad \mathcal{K}_{6}^{(s)}
$$

For type-II invariance their coefficients can have any $b_{\{2,4\}}^{(s)}\left(\hat{\omega}_{1}, \hat{\omega}_{2}\right)$, but require

$$
\begin{aligned}
& b_{1}^{(s)}\left(\hat{\omega}_{1}, \hat{\omega}-\omega_{1}\right)=C_{0}^{(s)}(\hat{\omega}), \quad b_{4}^{(s)}\left(\hat{\omega}, \hat{\omega}-\omega_{1}\right)=-2 \hat{\omega} \frac{d}{d \hat{\omega}} C_{0}^{(s)}(\hat{\omega}), \\
& b_{8}^{(s)}\left(\hat{\omega}_{1}, \hat{\omega}-\omega_{1}\right)=-2 \hat{\omega} \frac{d}{d \hat{\omega}} C_{0}^{(s)}(\hat{\omega})-\frac{2 \hat{\omega}}{\left(\hat{\omega}_{1}-\hat{\omega}\right)} C_{0}^{(s)}(\hat{\omega}) .
\end{aligned}
$$

The restrictions on the Wilson coefficients are summarized in Table I Comparing the invariants in Eqs. (61) and (67), we see that the combinations in Eq. (66) are the most general combinations invariant under type-I and type-II with the restrictions in Eq. (67) plus $b_{2}^{(s)}(\hat{\omega}, 0)=0$. However, with this constraint on $b_{2}^{(s)}$ the operator $\mathcal{K}_{2}^{(s)}$ is actually identical to $\mathcal{K}_{6}^{(s)}$ with an unconstrained coefficient $b_{6}^{(s)}$. To see this note that within square brackets $\left[W^{\dagger} i \overrightarrow{D D}_{c}^{\perp} W\right]_{\omega_{2}}=\left[W^{\dagger} i \overleftarrow{D D}_{c}^{\perp} W\right]_{\omega_{2}}=-\left[W^{\dagger} i g \not B_{c}^{\perp} W\right]_{\omega_{2}} / \omega_{2}$, so the difference comes from $i \overleftarrow{D D}_{c}^{\perp}$ acting also on $\left(\bar{\xi}_{n} W\right)$ in $\mathcal{K}_{2}^{(s)}$. However, since the factor $\left(\bar{\xi}_{n} W\right)$ on the left is a collinear color singlet we can write

$$
\left(W^{\dagger} i \overleftarrow{D}{ }_{c}^{\perp} W\right)_{\omega_{2}}=\left[W^{\dagger} i \overleftarrow{D D}_{c}^{\perp} W\right]_{\omega_{2}}-\mathcal{P}_{\perp}^{\dagger}\left(W^{\dagger} W\right)_{\omega_{2}}=\left[W^{\dagger} i \overleftarrow{D D}_{c}^{\perp} W\right]_{\omega_{2}}-\mathcal{P}_{\perp}^{\dagger} \delta\left(\omega_{2}\right)
$$

and the last term vanishes since $b_{2}\left(\omega_{1}, 0\right)=0$. Given this result and the constraints in 


\begin{tabular}{|c|c|c|c|}
\hline & $\begin{array}{c}\text { RPI-I } \\
b_{i}^{(s)}\left(\hat{\omega}_{1}, 0\right)=\end{array}$ & $\begin{array}{c}\text { RPI-II } \\
b_{i}^{(s)}\left(\hat{\omega}_{1}, \hat{\omega}_{2}\right)=\end{array}$ & Combined Constraints \\
\hline$b_{1}^{(s)}$ & $C_{0}^{(s)}\left(\hat{\omega}_{1}\right)$ & $C_{0}^{(s)}\left(\hat{\omega}_{1}+\hat{\omega}_{2}\right)$ & $b_{1}^{(s)}\left(\hat{\omega}_{1}, \hat{\omega}_{2}\right)=C_{0}^{(s)}\left(\hat{\omega}_{1}+\hat{\omega}_{2}\right)$ \\
\hline$b_{2}^{(s)}$ & 0 & $b_{2}^{(s)}\left(\hat{\omega}_{1}, \hat{\omega}_{2}\right)$ & $b_{2}^{(s)}\left(\hat{\omega}_{1}, 0\right)=0$ \\
\hline$b_{3}^{(s)}$ & 0 & 0 & $b_{3}^{(s)}\left(\hat{\omega}_{1}, \hat{\omega}_{2}\right)=0$ \\
\hline$b_{4}^{(s)}$ & $-2 \hat{\omega}_{1} C_{0}^{(s) \prime}\left(\hat{\omega}_{1}\right)$ & $-2\left(\hat{\omega}_{1}+\hat{\omega}_{2}\right) C_{0}^{(s)^{\prime}}\left(\hat{\omega}_{1}+\hat{\omega}_{2}\right)$ & $b_{4}^{(s)}\left(\hat{\omega}_{1}, \hat{\omega}_{2}\right)=-2\left(\hat{\omega}_{1}+\hat{\omega}_{2}\right) C_{0}^{(s)^{\prime}}\left(\hat{\omega}_{1}+\hat{\omega}_{2}\right)$ \\
\hline$b_{5}^{(s)}$ & $b_{5}^{(s)}\left(\hat{\omega}_{1}, 0\right)$ & 0 & $b_{5}^{(s)}\left(\hat{\omega}_{1}, \hat{\omega}_{2}\right)=0$ \\
\hline$b_{6}^{(s)}$ & $b_{6}^{(s)}\left(\hat{\omega}_{1}, 0\right)$ & $b_{6}^{(s)}\left(\hat{\omega}_{1}, \hat{\omega}_{2}\right)$ & $b_{6}^{(s)}\left(\hat{\omega}_{1}, \hat{\omega}_{2}\right)$ unconstrained \\
\hline$b_{7}^{(s)}$ & $b_{7}^{(s)}\left(\hat{\omega}_{1}, 0\right)$ & 0 & $b_{7}^{(s)}\left(\hat{\omega}_{1}, \hat{\omega}_{2}\right)=0$ \\
\hline \multirow[t]{2}{*}{$b_{8}^{(s)}$} & $b_{8}^{(s)}\left(\hat{\omega}_{1}, 0\right)$ & $-2\left(\hat{\omega}_{1}+\hat{\omega}_{2}\right) C_{0}^{(s)^{\prime}}\left(\hat{\omega}_{1}+\hat{\omega}_{2}\right)$ & $b_{8}^{(s)}\left(\hat{\omega}_{1}, \hat{\omega}_{2}\right)=-2\left(\hat{\omega}_{1}+\hat{\omega}_{2}\right) C_{0}^{(s)^{\prime}}\left(\hat{\omega}_{1}+\hat{\omega}_{2}\right)$ \\
\hline & & $+2\left(1+\frac{\hat{\omega}_{1}}{\hat{\omega}_{2}}\right) C_{0}^{(s)}\left(\hat{\omega}_{1}+\hat{\omega}_{2}\right)$ & $+2\left(1+\frac{\hat{\omega}_{1}}{\hat{\omega}_{2}}\right) C_{0}^{(s)}\left(\hat{\omega}_{1}+\hat{\omega}_{2}\right)$ \\
\hline
\end{tabular}

TABLE I: Summary of RPI constraints on the coefficients of the scalar currents in Eq. (566). The first column shows the constraints from type-I RPI on $b_{i}^{(s)}\left(\hat{\omega}_{1}, 0\right)$, the second column shows the constraint on $b_{i}^{(s)}\left(\hat{\omega}_{1}, \hat{\omega}_{2}\right)$ from type-II RPI and the third column gives the combined constraint. A generic entry, like $b_{2}^{(s)}\left(\hat{\omega}_{1}, \hat{\omega}_{2}\right)$ in the second row of the RPI-II column, indicates no constraint. The final currents are displayed in Eq. (71), and are defined so that they automatically satisfy these constraints.

Eq. (67) it is convenient to define

$$
\begin{aligned}
K_{1}^{(s)}(\omega) & =\int d \omega_{1} \mathcal{K}_{1}^{(s)}\left(\omega_{1}, \omega-\omega_{1}\right), \\
K_{2}^{(s)}(\omega) & =\hat{\omega} \int d \omega_{1}\left\{\mathcal{K}_{4}^{(s)}\left(\omega_{1}, \omega-\omega_{1}\right)+\mathcal{K}_{8}^{(s)}\left(\omega_{1}, \omega-\omega_{1}\right)\right\} \\
K_{3}^{(s)}(\omega) & =\omega \int d \omega_{1}\left(\omega_{1}-\omega\right)^{-1} \mathcal{K}_{8}^{(s)}\left(\omega_{1}, \omega-\omega_{1}\right), \\
K_{4}^{(s)}\left(\omega_{1}, \omega_{2}\right) & =\left(\hat{\omega}_{1}+\hat{\omega}_{2}\right) \mathcal{K}_{6}^{(s)}\left(\omega_{1}, \omega_{2}\right) .
\end{aligned}
$$

From RPI it is only these operators that can ever appear. RPI has ruled out some currents and restricted $K_{1,2,3}^{(s)}$ to only depend on one parameter. Once we know this, we can simply forget about the $\mathcal{K}_{i}^{(s)}$ and work directly with the $K_{i}^{(s)}$. Using capital $B$ 's for their Wilson coefficients, our final basis of subleading scalar operators $K_{1-4}^{(s)}$ is

$$
\begin{aligned}
K_{1-3}^{(s)} & =\int d \omega B_{1-3}^{(s)}(\hat{\omega}) \quad K_{1-3}^{(s)}(\omega), \\
K_{4}^{(s)} & =\int d \omega_{1} d \omega_{2} \quad B_{4}^{(s)}\left(\hat{\omega}_{1}, \hat{\omega}_{2}\right) \quad K_{4}^{(s)}\left(\omega_{1}, \omega_{2}\right),
\end{aligned}
$$


where

$$
\begin{aligned}
K_{1}^{(s)}(\omega) & =-\left(\bar{\xi}_{n} \frac{\not h}{2} i \overleftarrow{\not D}{ }_{c}^{\perp} W\right)_{\omega} \frac{1}{\overline{\mathcal{P}}^{\dagger}} h_{v} \\
K_{2}^{(s)}(\omega) & =\frac{v \cdot \mathcal{P}_{\perp}}{m_{b}}\left(\bar{\xi}_{n} W\right)_{\omega} h_{v} \\
K_{3}^{(s)}(\omega) & =\left(\bar{\xi}_{n} \frac{1}{n \cdot v i \bar{n} \cdot D_{c}} i v \cdot \vec{D}_{c}^{\perp} W\right)_{\omega} h_{v} \\
K_{4}^{(s)}\left(\omega_{1}, \omega_{2}\right) & =\frac{1}{m_{b}}\left(\bar{\xi}_{n} W\right)_{\omega_{1}}\left(\frac{1}{\overline{\mathcal{P}}} W^{\dagger} i g \mathbb{P}_{c}^{\perp} W\right)_{\omega_{2}} h_{v}
\end{aligned}
$$

and the RPI type-I and type-II constraints on the Wilson coefficients become

$$
B_{1}^{(s)}(\hat{\omega})=C_{0}^{(s)}(\hat{\omega}), \quad B_{2}^{(s)}(\hat{\omega})=-2 C_{0}^{(s) \prime}(\hat{\omega}), \quad B_{3}^{(s)}(\hat{\omega})=-2 C_{0}^{(s)}(\hat{\omega})
$$

The prime here denotes a derivative with respect to $\hat{\omega}$. Thus, we conclude that there are 4 subleading $\mathcal{O}(\lambda)$ scalar heavy-to-light currents. The coefficient $B_{4}^{(s)}\left(\hat{\omega}_{1}, \hat{\omega}_{2}\right)$ is completely unconstrained, while the other coefficients are fixed from RPI invariance.

In Ref. [13] it was noted that $J_{0}^{(s)}, K_{1}^{(s)}$, and $K_{3}^{(s)}$ are connected by RPI, and for these operators our results agree with taking their matching calculation in Eq. (120) and multiplying by a common Wilson coefficient. The operator $K_{2}^{(s)}$ does not appear in Ref. [13], because the derivative on its coefficient causes it to vanish at tree level. Our three-body operator $K_{4}^{(s)}$ is also new. In the limit that $B_{4}\left(\hat{\omega}_{1}, \hat{\omega}_{2}\right)$ depends only on the sum $\hat{\omega}_{+}=\hat{\omega}_{1}+\hat{\omega}_{2}$ we can switch variables to $\hat{\omega}_{+}, \hat{\omega}_{1}$ and reduce $K_{4}^{(s)}$ to a two-body operator. At tree level this is always possible since the Wilson coefficient is independent of the $\omega_{i}$. To see how the reduction works we write

$$
\begin{aligned}
\int d \omega_{1} K_{4}^{(s)}\left(\omega_{1}, \omega_{+}-\omega_{1}\right) & =\frac{1}{m_{b}}\left(\bar{\xi}_{n} W \frac{1}{\overline{\mathcal{P}}} W^{\dagger} i g \mathbb{P}_{c}^{\perp} W\right)_{\omega_{+}} h_{v} \\
& =\frac{1}{m_{b}}\left(\bar{\xi}_{n}\left[i \overrightarrow{D D}_{c}^{\perp} W\right]\right)_{\omega_{+}} h_{v},
\end{aligned}
$$

where in the last line we used Eq. (14). The derivative structure of this two-body operator is similar to that of operators in Ref. 12, 13], however the specific spin structure appearing in Eq. (73) does not appear from matching the QCD scalar current at tree level. Beyond tree level $B_{4}$ can depend separately on $\omega_{1}$ and $\omega_{2}$ and the reduction in Eq. (73) is not valid.

\section{B. Vector Currents}

The steps for deriving the general set of vector currents are very similar to the steps for the scalar currents in the previous section, so our presentation will be more concise.

At LO gauge invariance plus power counting allows currents of the form $\bar{\xi}_{n} W Z_{\mu} h_{v}$. Im- 
posing type-III RPI invariance allows the Dirac structures

$$
\begin{aligned}
& Z_{\mu}=\left\{\gamma_{\mu}, \gamma_{\mu} \not \bar{n} n \cdot v, \frac{\gamma_{\mu} \not \bar{n}}{\bar{n} \cdot v}, n_{\mu} \not \bar{h}, \frac{n_{\mu}}{n \cdot v}, n_{\mu} \bar{n} \cdot v, v_{\mu}, v_{\mu} \not \bar{n} n \cdot v, \frac{v_{\mu} \not \bar{h}}{\bar{n} \cdot v},\right.
\end{aligned}
$$

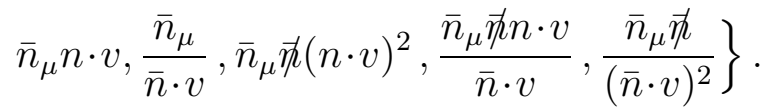

However it is easy to show that all structures involving $\bar{n}$ are ruled out by the $\delta_{\mathrm{II}}^{\left(\lambda^{0}\right)}$ transformations. Thus, at leading order there are only three allowed vector currents

$$
J_{1-3}^{(v)}=\int d \omega C_{1-3}^{(v)}(\hat{\omega}) \quad J_{1-3}^{(v)}(\omega),
$$

where the coefficients are functions of $\hat{\omega}=\omega / m_{b}$ and $\mu / m_{b}$, and

$$
J_{1-3}^{(v)}(\omega)=\left(\bar{\xi}_{n} W\right)_{\omega}\left\{\gamma_{\mu}, v_{\mu}, \frac{n_{\mu}}{n \cdot v}\right\} h_{v} .
$$

The choices $\{1,2,3\}$ correspond to the three different Dirac structures, and our basis in Eq. (76) agrees with Ref. [10].

At NLO the power counting only allows a single $D_{\perp}$ to appear. For the possible spin structures it is easy to see that type-II RPI invariance does not allow the vector index to be in an $\bar{n}^{\mu}$ or any factors of $\bar{n} \cdot v$ to appear just as for the leading currents. Imposing the constraints from gauge invariance, spin reduction, and type-III RPI then leaves $28 \mathcal{O}(\lambda)$ suppressed currents $[j=1, \ldots, 28]$

$$
\mathcal{K}_{j}^{(v)}=\int d \omega_{1} d \omega_{2} \quad b_{j}^{(v)}\left(\hat{\omega}_{1}, \hat{\omega}_{2}\right) \mathcal{K}_{j}^{(v)}\left(\omega_{1}, \omega_{2}\right)
$$

with operators

$$
\begin{aligned}
\mathcal{K}_{1-6}^{(v)}\left(\omega_{1}, \omega_{2}\right) & =\left(\bar{\xi}_{n} W\right)_{\omega_{1}}\left(W^{\dagger} i \overleftarrow{D}_{c}^{\perp} W\right)_{\omega_{2}} \frac{1}{\overline{\mathcal{P}}^{\dagger}} \Gamma_{1-6}^{\mu} h_{v}, \\
\mathcal{K}_{7,8}^{(v)}\left(\omega_{1}, \omega_{2}\right) & =\left(\bar{\xi}_{n} W\right)_{\omega_{1}}\left(W^{\dagger} i \overleftarrow{D}_{c}^{\perp \mu} W\right)_{\omega_{2}} \frac{1}{\overline{\mathcal{P}}^{\dagger}}\left\{\frac{\not h}{2}, \frac{1}{n \cdot v}\right\} h_{v} \\
\mathcal{K}_{9-14}^{(v)}\left(\omega_{1}, \omega_{2}\right) & =\left(\bar{\xi}_{n} W\right)_{\omega_{1}}\left(W^{\dagger} i v \cdot \overleftarrow{D}_{c}^{\perp} W\right)_{\omega_{2}} \frac{1}{\overline{\mathcal{P}}^{\dagger}} \Gamma_{1-6}^{\mu} h_{v}, \\
\mathcal{K}_{15-20}^{(v)}\left(\omega_{1}, \omega_{2}\right) & =\left(\bar{\xi}_{n} W\right)_{\omega_{1}}\left(W^{\dagger} i \vec{D}_{c}^{\perp} W\right)_{\omega_{2}} \frac{1}{\overline{\mathcal{P}}^{\dagger}} \Gamma_{1-6}^{\mu} h_{v}, \\
\mathcal{K}_{21,22}^{(v)}\left(\omega_{1}, \omega_{2}\right) & =\left(\bar{\xi}_{n} W\right)_{\omega_{1}}\left(W^{\dagger} i \vec{D}_{c}^{\perp \mu} W\right)_{\omega_{2}} \frac{1}{\overline{\mathcal{P}}^{\dagger}}\left\{\frac{\not h}{2}, \frac{1}{n \cdot v}\right\} h_{v}, \\
\mathcal{K}_{23-28}^{(v)}\left(\omega_{1}, \omega_{2}\right) & =\left(\bar{\xi}_{n} W\right)_{\omega_{1}}\left(W^{\dagger} i v \cdot \vec{D}_{c}^{\perp} W\right)_{\omega_{2}} \frac{1}{\overline{\mathcal{P}}^{\dagger}} \Gamma_{1-6}^{\mu} h_{v},
\end{aligned}
$$

where the six $\Gamma_{i}^{\mu}$ matrices are

$$
\Gamma_{1-6}^{\mu}=\left\{\frac{\not h \gamma^{\mu}}{2}, \frac{\not h v^{\mu}}{2}, \frac{\not h n^{\mu}}{2 n \cdot v}, \frac{\gamma^{\mu}}{n \cdot v}, \frac{v^{\mu}}{n \cdot v}, \frac{n^{\mu}}{(n \cdot v)^{2}}\right\} .
$$

Working out the transformations of the leading and subleading currents in a similar way as was done for the scalar currents we find that the type-I invariants are $J_{1}^{(v)}+\mathcal{K}_{1}^{(v)}+\mathcal{K}_{12}^{(v)}$, 


\begin{tabular}{|c|c|c|c|}
\hline & $\begin{array}{c}\text { RPI-I } \\
b_{i}^{(v)}\left(\hat{\omega}_{1}, 0\right)=\end{array}$ & $\begin{array}{c}\text { RPI-II } \\
b_{i}^{(v)}\left(\hat{\omega}_{1}, \hat{\omega}_{2}\right)=\end{array}$ & Combined Constraints \\
\hline$b_{1-3}^{(v)}$ & $C_{1-3}^{(v)}\left(\hat{\omega}_{1}\right)$ & $C_{1-3}^{(v)}\left(\hat{\omega}_{1}+\hat{\omega}_{2}\right)$ & $b_{1-3}^{(v)}\left(\hat{\omega}_{1}, \hat{\omega}_{2}\right)=C_{1-3}^{(v)}\left(\hat{\omega}_{1}+\hat{\omega}_{2}\right)$ \\
\hline$b_{4-6}^{(v)}$ & 0 & $b_{4-6}^{(v)}\left(\hat{\omega}_{1}, \hat{\omega}_{2}\right)$ & $b_{4-6}^{(v)}\left(\hat{\omega}_{1}, 0\right)=0$ \\
\hline$b_{7}^{(v)}$ & 0 & 0 & $b_{7}^{(v)}\left(\hat{\omega}_{1}, \hat{\omega}_{2}\right)=0$ \\
\hline$b_{8}^{(v)}$ & $-2 C_{3}^{(v)}\left(\hat{\omega}_{1}\right)$ & $b_{8}^{(v)}\left(\hat{\omega}_{1}, \hat{\omega}_{2}\right)$ & $b_{8}^{(v)}\left(\hat{\omega}_{1}, 0\right)=-2 C_{3}^{(v)}\left(\hat{\omega}_{1}\right)$ \\
\hline$b_{9-11}^{(v)}$ & 0 & 0 & $b_{9-11}^{(v)}\left(\hat{\omega}_{1}, \hat{\omega}_{2}\right)=0$ \\
\hline$b_{12,13}^{(v)}$ & $-2 \hat{\omega}_{1} C_{1,2}^{(v) \prime}\left(\hat{\omega}_{1}\right)$ & $-2\left(\hat{\omega}_{1}+\hat{\omega}_{2}\right) C_{1,2}^{(v)^{\prime}}\left(\hat{\omega}_{1}+\hat{\omega}_{2}\right)$ & $b_{12,13}^{(v)}\left(\hat{\omega}_{1}, \hat{\omega}_{2}\right)=-2\left(\hat{\omega}_{1}+\hat{\omega}_{2}\right) C_{1,2}^{(v)^{\prime}}\left(\hat{\omega}_{1}+\hat{\omega}_{2}\right)$ \\
\hline \multirow[t]{2}{*}{$b_{14}^{(v)}$} & $-2 \hat{\omega}_{1} C_{3}^{(v) \prime}\left(\hat{\omega}_{1}\right)$ & $-2\left(\hat{\omega}_{1}+\hat{\omega}_{2}\right) C_{3}^{(v)^{\prime}}\left(\hat{\omega}_{1}+\hat{\omega}_{2}\right)$ & $b_{14}^{(v)}\left(\hat{\omega}_{1}, \hat{\omega}_{2}\right)=-2\left(\hat{\omega}_{1}+\hat{\omega}_{2}\right) C_{3}^{(v)^{\prime}}\left(\hat{\omega}_{1}+\hat{\omega}_{2}\right)$ \\
\hline & $+2 C_{3}^{(v)}\left(\hat{\omega}_{1}\right)$ & $-b_{8}^{(v)}\left(\hat{\omega}_{1}, \hat{\omega}_{2}\right)$ & $-b_{8}^{(v)}\left(\hat{\omega}_{1}, \hat{\omega}_{2}\right)$ \\
\hline$b_{15-17}^{(v)}$ & $b_{15-17}^{(v)}\left(\hat{\omega}_{1}, 0\right)$ & 0 & $b_{15-17}^{(v)}\left(\hat{\omega}_{1}, \hat{\omega}_{2}\right)=0$ \\
\hline$b_{18-20}^{(v)}$ & $b_{18-20}^{(v)}\left(\hat{\omega}_{1}, 0\right)$ & $b_{18-20}^{(v)}\left(\hat{\omega}_{1}, \hat{\omega}_{2}\right)$ & $b_{18-20}^{(v)}\left(\hat{\omega}_{1}, \hat{\omega}_{2}\right)$ unconstrained \\
\hline$b_{21}^{(v)}$ & $b_{21}^{(v)}\left(\hat{\omega}_{1}, 0\right)$ & 0 & $b_{21}^{(v)}\left(\hat{\omega}_{1}, \hat{\omega}_{2}\right)=0$ \\
\hline$b_{22}^{(v)}$ & $b_{22}^{(v)}\left(\hat{\omega}_{1}, 0\right)$ & $b_{22}^{(v)}\left(\hat{\omega}_{1}, \hat{\omega}_{2}\right)$ & $b_{22}^{(v)}\left(\hat{\omega}_{1}, \hat{\omega}_{2}\right)$ unconstrained \\
\hline$b_{23-25}^{(v)}$ & $b_{23-25}^{(v)}\left(\hat{\omega}_{1}, 0\right)$ & 0 & $b_{23-25}^{(v)}\left(\hat{\omega}_{1}, \hat{\omega}_{2}\right)=0$ \\
\hline \multirow[t]{2}{*}{$b_{26,27}^{(v)}$} & $b_{26,27}^{(v)}\left(\hat{\omega}_{1}, 0\right)$ & $-2\left(\hat{\omega}_{1}+\hat{\omega}_{2}\right) C_{1,2}^{(v)^{\prime}}\left(\hat{\omega}_{1}+\hat{\omega}_{2}\right)$ & $b_{26,27}^{(v)}\left(\hat{\omega}_{1}, \hat{\omega}_{2}\right)=-2\left(\hat{\omega}_{1}+\hat{\omega}_{2}\right) C_{1,2}^{(v)^{\prime}}\left(\hat{\omega}_{1}+\hat{\omega}_{2}\right)$ \\
\hline & & $-2 C_{1,2}^{(v)}\left(\hat{\omega}_{1}+\hat{\omega}_{2}\right)$ & $-2 C_{1,2}^{(v)}\left(\hat{\omega}_{1}+\hat{\omega}_{2}\right)$ \\
\hline \multirow[t]{3}{*}{$b_{28}^{(v)}$} & $b_{28}^{(v)}\left(\hat{\omega}_{1}, 0\right)$ & $-2\left(\hat{\omega}_{1}+\hat{\omega}_{2}\right) C_{3}^{(v)^{\prime}}\left(\hat{\omega}_{1}+\hat{\omega}_{2}\right)$ & $b_{28}^{(v)}\left(\hat{\omega}_{1}, \hat{\omega}_{2}\right)=-2\left(\hat{\omega}_{1}+\hat{\omega}_{2}\right) C_{3}^{(v)^{\prime}}\left(\hat{\omega}_{1}+\hat{\omega}_{2}\right)$ \\
\hline & & $+2\left(1+\frac{\hat{\omega}_{1}}{\hat{\omega}_{2}}\right) C_{3}^{(v)}\left(\hat{\omega}_{1}+\hat{\omega}_{2}\right)$ & $+2\left(1+\frac{\hat{\omega}_{1}}{\hat{\omega}_{2}}\right) C_{3}^{(v)}\left(\hat{\omega}_{1}+\hat{\omega}_{2}\right)$ \\
\hline & & $-b_{22}\left(\hat{\omega}_{1}, \hat{\omega}_{2}\right)$ & $-b_{22}\left(\hat{\omega}_{1}, \hat{\omega}_{2}\right)$ \\
\hline
\end{tabular}

TABLE II: Summary of RPI constraints on the coefficients of the vector currents in Eq. (78). The first column shows the constraints from type-I RPI on $b_{i}^{(s)}(\omega, 0)$, the second column shows the constraint on $b_{i}^{(s)}\left(\omega_{1}, \omega_{2}\right)$ from type-II RPI and the third column gives the combined constraint. The final currents are displayed in Eq. (81), and are defined so that they automatically satisfy these constraints.

$J_{2}^{(v)}+\mathcal{K}_{2}^{(v)}+\mathcal{K}_{13}^{(v)}, J_{3}^{(v)}+\mathcal{K}_{3}^{(v)}+\mathcal{K}_{8}^{(v)}+\mathcal{K}_{14}^{(v)}, \mathcal{K}_{j}^{(v)}, \mathcal{K}_{k}^{(v)}$, where $j=\{4,5,6,7,9,10,11\}$ and $k=\{15, \ldots, 28\}$. Type-I invariance allows any coefficients $b_{k}^{(v)}\left(\hat{\omega}_{1}, \hat{\omega}_{2}\right)$, but restricts $b_{1-14}^{(v)}\left(\hat{\omega}_{1}, \hat{\omega}_{2}\right)$ as shown in the second column of Table 【.

Looking at the transformations under type-II we find that the invariants are independent of the $\left\{\gamma_{\mu}, v_{\mu}, n_{\mu}\right\}$ choice. Our results for the type-II invariants are $J_{1}^{(v)}+\mathcal{K}_{1}^{(v)}+\mathcal{K}_{12}^{(v)}+\mathcal{K}_{26}^{(v)}$, $J_{2}^{(v)}+\mathcal{K}_{2}^{(v)}+\mathcal{K}_{13}^{(v)}+\mathcal{K}_{27}^{(v)}, J_{3}^{(v)}+\mathcal{K}_{3}^{(v)}+\mathcal{K}_{8}^{(v)}+\mathcal{K}_{14}^{(v)}+\left(\mathcal{K}_{22}^{(v)}+\mathcal{K}_{28}^{(v)}\right),\left(\mathcal{K}_{22}^{(v)}-\mathcal{K}_{28}^{(v)}\right)$, while $\mathcal{K}_{\ell}^{(v)}$ for $\ell=\{4,5,6,18,19,20\}$ are invariant by themselves. For these combinations type-II 
invariance allows any $b_{\ell}^{(v)}\left(\hat{\omega}_{1}, \hat{\omega}_{2}\right)$, but restricts $b_{1-3,7,9-17,21-28}^{(v)}$ as shown in the third column of Table II. Furthermore, currents $\mathcal{K}_{m}^{(v)}$ with $m=\{7,9,10,11,15,16,17,21,23,24,25\}$ are ruled out (ie. $\left.b_{m}^{(v)}=0\right)$.

It is easy to see that the type-I and type-II conditions in Table 1 are compatible. The combined set of constraints are given by those in the fourth column. Using Eq. (68) we can show that the constrained $\mathcal{K}_{\{4,5,6\}}^{(v)}$ are redundant with $\mathcal{K}_{\{18,19,20\}}^{(v)}$ respectively, just as was done for the scalar current with $\mathcal{K}_{2}^{(s)}$ and $\mathcal{K}_{6}^{(s)}$. We can also use Eq. (68) to convert $K_{8}^{(v)}\left(\hat{\omega}_{1}, \hat{\omega}_{2}\right)-K_{14}^{(v)}\left(\hat{\omega}_{1}, \hat{\omega}_{2}\right)$ into a term proportional to $\delta\left(\omega_{2}\right)$ and a term that is redundant with $K_{22}^{(v)}\left(\hat{\omega}_{1}, \hat{\omega}_{2}\right)-K_{28}^{(v)}\left(\hat{\omega}_{1}, \hat{\omega}_{2}\right)$.

From the combined constraints we can then define a new complete set of allowed vector operators, $K_{1-14}^{(v)}$. Therefore after imposing type-I and type-II RPI plus all other constraints we are left with our final set of allowed vector current operators

$$
\begin{aligned}
K_{1-10}^{(v)} & =\int d \omega B_{1-10}^{(v)}(\hat{\omega}) K_{1-10}^{(v)}(\omega), \\
K_{11-14}^{(v)} & =\int d \omega_{1} d \omega_{2} B_{11-14}^{(v)}\left(\hat{\omega}_{1}, \hat{\omega}_{2}\right) K_{11-14}^{(v)}\left(\omega_{1}, \omega_{2}\right),
\end{aligned}
$$

where

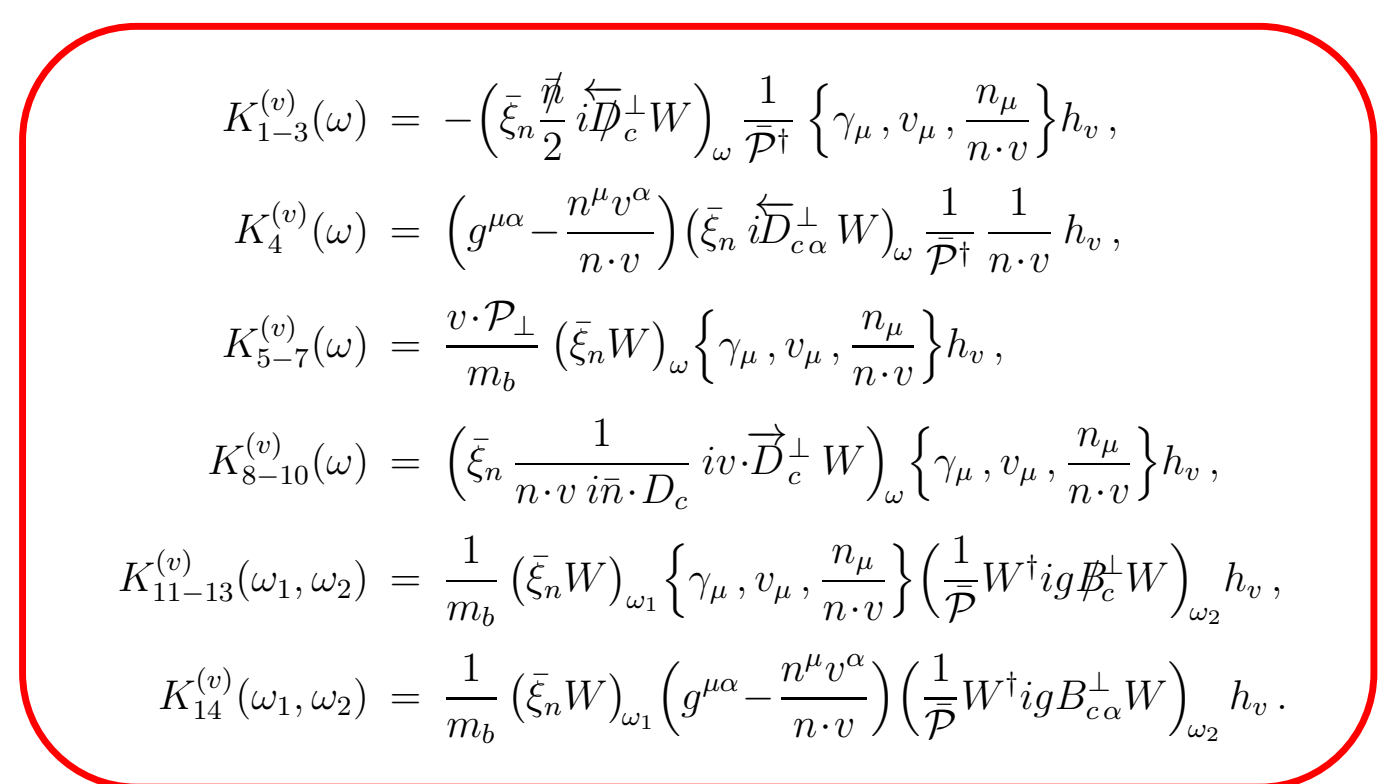

The coefficients $B_{\{11,12,13,14\}}^{(v)}$ in Eq. (80) depend on two parameters $\omega_{1,2}$ and are unconstrained. The remaining coefficients depend on only one parameter and are fixed by reparameterization invariance

$$
\begin{aligned}
B_{1-3}^{(v)}(\hat{\omega}) & =C_{1-3}^{(v)}(\hat{\omega}), \quad B_{5-7}^{(v)}(\hat{\omega})=-2 C_{1-3}^{(v) \prime}(\hat{\omega}), \quad B_{8-10}^{(v)}(\hat{\omega})=-2 C_{1-3}^{(v)}(\hat{\omega}), \\
B_{4}^{(v)}(\hat{\omega}) & =-2 C_{3}^{(v)}(\hat{\omega}) .
\end{aligned}
$$

The form of the currents $K_{1,2}^{(v)}$ agree with Ref. [10], and if we take a frame where $n \cdot v=1$ and $v_{\perp}=0$ then $K_{3,4}^{(v)}$ also agree. Ref. [10] looked at type-I RPI of the vector currents 
and our constraints on $B_{1-4}^{(v)}$ agree with the ones found there. (We note that the authors of Ref. [10] also checked these results with explicit one-loop computations.) At tree-level one matches onto the currents $K_{18}^{(v)}$ and the two-body limit of $K_{13}^{(v)}$ (using the analog of Eq. (73) $)$, and we agree with Ref. [13] on the form of these currents and the RPI constraint between $K_{1}^{(v)}$ and $K_{8}^{(v)}$. The structures in Eq. (81) which are new and which only appear beyond tree level are $K_{5-7,9-12,14}^{(v)}$ and the three-body form of $K_{13}^{(v)}$.

\section{Pseudoscalar and Axial-Vector Currents}

The results for the pseudoscalar and axial-vector heavy-to-light currents can be directly obtained from the analysis for the scalar and vector currents respectively. The analysis is identical except for the extra $\gamma_{5}$ in the Dirac structure. For the pseudo-scalar currents we have the basis $\{\not h / 2,1 / n \cdot v\} \gamma_{5}$, while for the axial-vector currents we have $\Gamma_{\{1, \ldots, 6\}}^{\mu} \gamma_{5}$ where $\Gamma_{j}^{\mu}$ is defined in Eq. (179). At LO the most general allowed pseudoscalar current are thus

$$
\begin{aligned}
J_{0}^{(p)} & =\int d \omega C_{0}^{(p)}\left(\hat{\omega}, \mu / m_{b}\right) J_{0}^{(p)}(\omega), \\
J_{0}^{(p)}(\omega) & =\left(\bar{\xi}_{n} W\right)_{\omega_{1}} \gamma_{5} h_{v},
\end{aligned}
$$

while the axial-vector currents are

$$
\begin{aligned}
J_{1-3}^{(a)} & =\int d \omega C_{1-3}^{(a)}(\hat{\omega}) J_{1-3}^{(a)}(\omega), \\
J_{1-3}^{(a)}(\omega) & =\left(\bar{\xi}_{n} W\right)_{\omega}\left\{\gamma_{\mu},-v_{\mu},-\frac{n_{\mu}}{n \cdot v}\right\} \gamma_{5} h_{v} .
\end{aligned}
$$

At NLO we again have eight possible pseudo-scalar currents $\mathcal{K}_{j}^{(p)}$ and 28 possible axialvector currents $\mathcal{K}_{j}^{(a)}$ before imposing all type-I and type-II constraints. After imposing the RPI constraints we find results very similar to those in Eq. (71) and (81). Thus for the final NLO pseudoscalar currents we have

$$
\begin{aligned}
K_{1-3}^{(p)} & =\int d \omega B_{1-3}^{(p)}(\hat{\omega}) K_{1-3}^{(p)}(\omega) \\
K_{4}^{(p)} & =\int d \omega_{1} d \omega_{2} B_{4}^{(p)}\left(\hat{\omega}_{1}, \hat{\omega}_{2}\right) \quad K_{4}^{(p)}\left(\omega_{1}, \omega_{2}\right),
\end{aligned}
$$

where 


$$
\begin{aligned}
K_{1}^{(p)}(\omega) & =-\left(\bar{\xi}_{n} \frac{\not h}{2} i \overleftarrow{D}_{c}^{\perp} W\right)_{\omega} \frac{1}{\overline{\mathcal{P}}^{\dagger}} \gamma_{5} h_{v} \\
K_{2}^{(p)}(\omega) & =\frac{v \cdot \mathcal{P}_{\perp}}{m_{b}}\left(\bar{\xi}_{n} W\right)_{\omega} \gamma_{5} h_{v} \\
K_{3}^{(p)}(\omega) & =\left(\bar{\xi}_{n} \frac{1}{n \cdot v i \bar{n} \cdot D_{c}} i v \cdot \vec{D}_{c}^{\perp} W\right)_{\omega} \gamma_{5} h_{v} \\
K_{4}^{(p)}\left(\omega_{1}, \omega_{2}\right) & =\frac{1}{m_{b}}\left(\bar{\xi}_{n} W\right)_{\omega_{1}} \gamma_{5}\left(\frac{1}{\overline{\mathcal{P}}} W^{\dagger} i g \mathbb{B}_{c}^{\perp} W\right)_{\omega_{2}} h_{v}
\end{aligned}
$$

and the RPI type-I and type-II constraints on the Wilson coefficients are

$$
B_{1}^{(p)}(\hat{\omega})=C_{0}^{(p)}(\hat{\omega}), \quad B_{2}^{(p)}(\hat{\omega})=-2 C_{0}^{(p) \prime}(\hat{\omega}), \quad B_{3}^{(p)}(\hat{\omega})=-2 C_{0}^{(p)}(\hat{\omega}) .
$$

For the final axial-vector NLO currents we find

$$
\begin{aligned}
K_{1-10}^{(a)} & =\int d \omega \quad B_{1-10}^{(a)}(\hat{\omega}) K_{1-10}^{(a)}(\omega), \\
K_{11-14}^{(a)} & =\int d \omega_{1} d \omega_{2} B_{11-14}^{(a)}\left(\hat{\omega}_{1}, \hat{\omega}_{2}\right) \quad K_{11-14}^{(a)}\left(\omega_{1}, \omega_{2}\right),
\end{aligned}
$$

where

$$
\begin{aligned}
K_{1-3}^{(a)}(\omega) & =-\left(\bar{\xi}_{n} \frac{\not h}{2} i \overleftarrow{D D}_{c}^{\perp} W\right)_{\omega} \frac{1}{\overline{\mathcal{P}}^{\dagger}}\left\{\gamma_{\mu},-v_{\mu},-\frac{n_{\mu}}{n \cdot v}\right\} \gamma_{5} h_{v} \\
K_{4}^{(a)}(\omega) & =-\left(g^{\mu \alpha}-\frac{n^{\mu} v^{\alpha}}{n \cdot v}\right)\left(\bar{\xi}_{n} i \overleftarrow{D}_{c \alpha}^{\perp} W\right)_{\omega} \frac{1}{\overline{\mathcal{P}}^{\dagger}} \frac{1}{n \cdot v} \gamma_{5} h_{v} \\
K_{5-7}^{(a)}(\omega) & =\frac{v \cdot \mathcal{P}_{\perp}}{m_{b}}\left(\bar{\xi}_{n} W\right)_{\omega}\left\{\gamma_{\mu},-v_{\mu},-\frac{n_{\mu}}{n \cdot v}\right\} \gamma_{5} h_{v} \\
K_{8-10}^{(a)}(\omega) & =\left(\bar{\xi}_{n} \frac{1}{n \cdot v i \bar{n} \cdot D_{c}} i v \cdot \vec{D}_{c}^{\perp} W\right)_{\omega}\left\{\gamma_{\mu},-v_{\mu},-\frac{n_{\mu}}{n \cdot v}\right\} \gamma_{5} h_{v} \\
K_{11-13}^{(a)}\left(\omega_{1}, \omega_{2}\right) & =\frac{1}{m_{b}}\left(\bar{\xi}_{n} W\right)_{\omega_{1}}\left\{\gamma_{\mu},-v_{\mu},-\frac{n_{\mu}}{n \cdot v}\right\} \gamma_{5}\left(\frac{1}{\overline{\mathcal{P}}} W^{\dagger} i g \mathbb{B}_{c}^{\perp} W\right)_{\omega_{2}} h_{v} \\
K_{14}^{(a)} & =-\frac{1}{m_{b}}\left(\bar{\xi}_{n} W\right)_{\omega_{1}}\left(g^{\mu \alpha}-\frac{n^{\mu} v^{\alpha}}{n \cdot v}\right) \gamma_{5}\left(\frac{1}{\overline{\mathcal{P}}} W^{\dagger} i g B_{c \alpha}^{\perp} W\right)_{\omega_{2}} h_{v}
\end{aligned}
$$

The coefficients $B_{\{11,12,13,14\}}^{(a)}$ in Eq. (80) depend on two parameters $\omega_{1,2}$ and are unconstrained. The remaining coefficients depend on only one parameter and are fixed by reparameterization invariance

$$
\begin{aligned}
B_{1-3}^{(a)}(\hat{\omega}) & =C_{1-3}^{(a)}(\hat{\omega}), \quad B_{5-7}^{(a)}(\hat{\omega})=-2 C_{1-3}^{(a) \prime}(\hat{\omega}), \quad B_{8-10}^{(a)}(\hat{\omega})=-2 C_{1-3}^{(a)}(\hat{\omega}), \\
B_{4}^{(a)}(\hat{\omega}) & =-2 C_{2}^{(a)}(\hat{\omega}) .
\end{aligned}
$$


The form of the pseudo-scalar and axial-vector currents are very analogous to the scalar and vector currents, so rather than comparing with the literature we simply refer to the comparisons in the proceeding sections for which part of our results were previously known.

\section{Tensor Currents}

At leading order in $\lambda$, there are four tensor currents, defined as

$$
J_{1-4}^{\mu \nu}(\omega)=\left(\bar{\xi}_{n} W\right)_{\omega} \Gamma_{1-4}^{\mu \nu} h_{v},
$$

where the most general allowed Dirac structures are

$$
\Gamma_{1-4}^{\mu \nu}=\left\{i \sigma^{\mu \nu}, \gamma^{[\mu,} v^{\nu]}, \frac{1}{n \cdot v} \gamma^{[\mu,} n^{\nu]}, \frac{1}{n \cdot v} n^{[\mu,} v^{\nu]}\right\},
$$

where $\gamma_{[\mu}, v_{\nu]}=\gamma_{\mu} v_{\nu}-\gamma_{\nu} v_{\mu}$ etc. As before, no $\bar{n}_{\mu}$ can appear at leading order from type-II RPI.

At $O(\lambda), 44$ currents can be written down before imposing the RPI constraints. They can be chosen as

$$
\begin{aligned}
\mathcal{K}_{1-8}^{(t)} & =\left(\bar{\xi}_{n} W\right)_{\omega_{1}}\left(W^{\dagger} i \overleftarrow{\not D}{ }_{c}^{\perp} W\right)_{\omega_{2}}\left\{\frac{\not h}{2} \Gamma_{1-4}^{\mu \nu}, \frac{1}{n \cdot v} \Gamma_{1-4}^{\mu \nu}\right\} \frac{1}{\overline{\mathcal{P}}^{\dagger}} h_{v}, \\
\mathcal{K}_{9-14}^{(t)} & =\left(\bar{\xi}_{n} W\right)_{\omega_{1}}\left(W^{\dagger} i \overleftarrow{D}_{c \perp}^{[\mu,} W\right)_{\omega_{2}} \Gamma_{1-6}^{\nu]} \frac{1}{\overline{\mathcal{P}}^{\dagger}} h_{v}, \\
\mathcal{K}_{15-22}^{(t)} & =\left(\bar{\xi}_{n} W\right)_{\omega_{1}}\left(W^{\dagger} i v \cdot \overleftarrow{D} \overleftarrow{c}_{c}^{\perp} W\right)_{\omega_{2}}\left\{\frac{\not h}{2} \Gamma_{1-4}^{\mu \nu}, \frac{1}{n \cdot v} \Gamma_{1-4}^{\mu \nu}\right\} \frac{1}{\overline{\mathcal{P}}^{\dagger}} h_{v},
\end{aligned}
$$

and

$$
\begin{aligned}
& \mathcal{K}_{23-30}^{(t)}=\left(\bar{\xi}_{n} W\right)_{\omega_{1}}\left(W^{\dagger} i \vec{D}_{c}^{\perp} W\right)_{\omega_{2}}\left\{\frac{\not h}{2} \Gamma_{1-4}^{\mu \nu}, \frac{1}{n \cdot v} \Gamma_{1-4}^{\mu \nu}\right\} \frac{1}{\overline{\mathcal{P}}^{\dagger}} h_{v}, \\
& \mathcal{K}_{31-36}^{(t)}=\left(\bar{\xi}_{n} W\right)_{\omega_{1}}\left(W^{\dagger} i \vec{D}_{c \perp}^{[\mu,} W\right)_{\omega_{2}} \Gamma_{1-6}^{\nu]} \frac{1}{\overline{\mathcal{P}}^{\dagger}} h_{v} \\
& \mathcal{K}_{37-44}^{(t)}=\left(\bar{\xi}_{n} W\right)_{\omega_{1}}\left(W^{\dagger} i v \cdot \vec{D}_{c}^{\perp} W\right)_{\omega_{2}}\left\{\frac{\not \vec{h}}{2} \Gamma_{1-4}^{\mu \nu}, \frac{1}{n \cdot v} \Gamma_{1-4}^{\mu \nu}\right\} \frac{1}{\overline{\mathcal{P}}^{\dagger}} h_{v} .
\end{aligned}
$$

The Dirac matrix with one index $\Gamma_{1-6}^{\mu}$ is defined as in (79)

$$
\Gamma_{1-6}^{\mu}=\left\{\frac{\not h}{2}\left(\gamma_{\mu}, v_{\mu}, \frac{n_{\mu}}{v \cdot n}\right), \frac{1}{n \cdot v}\left(\gamma_{\mu}, v_{\mu}, \frac{n_{\mu}}{v \cdot n}\right)\right\}
$$

The constraints from type-I and type-II RPI are derived as before. The final constraints on the Wilson coefficients $b_{1-44}$ are shown in Table [II]

After imposing the constraints from the table one finds the final minimal set of tensor heavy-light currents in the effective theory at $O(\lambda)$

$$
\begin{aligned}
K_{1-14}^{(t)} & =\int \mathrm{d} \omega B_{1-14}^{(t)}(\omega) K_{1-14}^{\mu \nu}(\omega), \\
K_{15-21}^{(t)} & =\int \mathrm{d} \omega_{1} \mathrm{~d} \omega_{2} B_{15-21}^{(t)}\left(\omega_{1}, \omega_{2}\right) K_{15-21}^{\mu \nu}\left(\omega_{1}, \omega_{2}\right) .
\end{aligned}
$$




\begin{tabular}{|c|c|c|c|}
\hline & $\begin{array}{c}\text { RPI-I } \\
b_{i}^{(t)}\left(\hat{\omega}_{1}, 0\right)=\end{array}$ & $\begin{array}{c}\text { RPI-II } \\
b_{i}^{(t)}\left(\hat{\omega}_{1}, \hat{\omega}_{2}\right)=\end{array}$ & Combined Constraints \\
\hline$b_{1-4}^{(t)}$ & $C_{1-4}^{(t)}\left(\hat{\omega}_{1}\right)$ & $C_{1-4}^{(t)}\left(\hat{\omega}_{1}+\hat{\omega}_{2}\right)$ & $b_{1-4}^{(t)}\left(\hat{\omega}_{1}, \hat{\omega}_{2}\right)=C_{1-4}^{(t)}\left(\hat{\omega}_{1}+\hat{\omega}_{2}\right)$ \\
\hline$b_{5-8}^{(t)}$ & 0 & $b_{5-8}^{(t)}\left(\hat{\omega}_{1}, \hat{\omega}_{2}\right)$ & $b_{5-8}^{(t)}\left(\hat{\omega}_{1}, 0\right)=0$ \\
\hline$b_{9-11}^{(t)}$ & 0 & 0 & $b_{9-11}^{(t)}\left(\hat{\omega}_{1}, \hat{\omega}_{2}\right)=0$ \\
\hline$b_{12}^{(t)}$ & $2 C_{3}^{(t)}\left(\hat{\omega}_{1}\right)$ & $b_{12}^{(t)}\left(\hat{\omega}_{1}, \hat{\omega}_{2}\right)$ & $b_{12}^{(t)}\left(\hat{\omega}_{1}, 0\right)=2 C_{3}^{(t)}\left(\hat{\omega}_{1}\right)$ \\
\hline$b_{13}^{(t)}$ & $-2 C_{4}^{(t)}\left(\hat{\omega}_{1}\right)$ & $b_{13}^{(t)}\left(\hat{\omega}_{1}, \hat{\omega}_{2}\right)$ & $b_{13}^{(t)}\left(\hat{\omega}_{1}, 0\right)=-2 C_{4}^{(t)}\left(\hat{\omega}_{1}\right)$ \\
\hline$b_{14}^{(t)}$ & 0 & $b_{14}^{(t)}\left(\hat{\omega}_{1}, \hat{\omega}_{2}\right)$ & $b_{14}^{(t)}\left(\hat{\omega}_{1}, 0\right)=0$ \\
\hline$b_{15-18}^{(t)}$ & 0 & 0 & $b_{15-18}^{(t)}\left(\hat{\omega}_{1}, \hat{\omega}_{2}\right)=0$ \\
\hline$b_{19,20}^{(t)}$ & $-2 \hat{\omega}_{1} C_{1,2}^{(t) \prime}\left(\hat{\omega}_{1}\right)$ & $-2\left(\hat{\omega}_{1}+\hat{\omega}_{2}\right) C_{1,2}^{(t)^{\prime}}\left(\hat{\omega}_{1}+\hat{\omega}_{2}\right)$ & $b_{19,20}^{(t)}\left(\hat{\omega}_{1}, \hat{\omega}_{2}\right)=-2\left(\hat{\omega}_{1}+\hat{\omega}_{2}\right) C_{1,2}^{(t)^{\prime}}\left(\hat{\omega}_{1}+\hat{\omega}_{2}\right)$ \\
\hline \multirow[t]{2}{*}{$b_{21,22}^{(t)}$} & $-2 \hat{\omega}_{1} C_{3,4}^{(t) \prime}\left(\hat{\omega}_{1}\right)$ & $-2\left(\hat{\omega}_{1}+\hat{\omega}_{2}\right) C_{3,4}^{(t)^{\prime}}\left(\hat{\omega}_{1}+\hat{\omega}_{2}\right)$ & $b_{21,22}^{(t)}\left(\hat{\omega}_{1}, \hat{\omega}_{2}\right)=-2\left(\hat{\omega}_{1}+\hat{\omega}_{2}\right) C_{3,4}^{(t)^{\prime}}\left(\hat{\omega}_{1}+\hat{\omega}_{2}\right)$ \\
\hline & $+2 C_{3,4}^{(t)}\left(\hat{\omega}_{1}\right)$ & $\pm b_{12,13}^{(t)}\left(\hat{\omega}_{1}, \hat{\omega}_{2}\right)$ & $\pm b_{12,13}^{(t)}\left(\hat{\omega}_{1}, \hat{\omega}_{2}\right)$ \\
\hline$b_{23-26}^{(t)}$ & $b_{23-26}^{(t)}\left(\hat{\omega}_{1}, 0\right)$ & 0 & $b_{23-26}^{(t)}\left(\hat{\omega}_{1}, \hat{\omega}_{2}\right)=0$ \\
\hline$b_{27-30}^{(t)}$ & $b_{27-30}^{(t)}\left(\hat{\omega}_{1}, 0\right)$ & $b_{27-30}^{(t)}\left(\hat{\omega}_{1}, \hat{\omega}_{2}\right)$ & $b_{27-30}^{(t)}\left(\hat{\omega}_{1}, \hat{\omega}_{2}\right)$ unconstrained \\
\hline$b_{31-33}^{(t)}$ & $b_{31-33}^{(t)}\left(\hat{\omega}_{1}, 0\right)$ & 0 & $b_{31-33}^{(t)}\left(\hat{\omega}_{1}, \hat{\omega}_{2}\right)=0$ \\
\hline$b_{34-36}^{(t)}$ & $b_{34-36}^{(t)}\left(\hat{\omega}_{1}, 0\right)$ & $b_{34-36}^{(t)}\left(\hat{\omega}_{1}, \hat{\omega}_{2}\right)$ & $b_{34-36}^{(t)}\left(\hat{\omega}_{1}, \hat{\omega}_{2}\right)$ unconstrained \\
\hline$b_{37-40}^{(t)}$ & $b_{37-40}^{(t)}\left(\hat{\omega}_{1}, 0\right)$ & 0 & $b_{37-40}^{(t)}\left(\hat{\omega}_{1}, \hat{\omega}_{2}\right)=0$ \\
\hline \multirow[t]{2}{*}{$b_{41,42}^{(t)}$} & $b_{41,42}^{(t)}\left(\hat{\omega}_{1}, 0\right)$ & $-2\left(\hat{\omega}_{1}+\hat{\omega}_{2}\right) C_{1,2}^{(t)^{\prime}}\left(\hat{\omega}_{1}+\hat{\omega}_{2}\right)$ & $b_{41,42}^{(t)}\left(\hat{\omega}_{1}, \hat{\omega}_{2}\right)=-2\left(\hat{\omega}_{1}+\hat{\omega}_{2}\right) C_{1,2}^{(t)^{\prime}}\left(\hat{\omega}_{1}+\hat{\omega}_{2}\right)$ \\
\hline & & $+2\left(1+\frac{\hat{\omega}_{1}}{\hat{\omega}_{2}}\right) C_{1,2}^{(t)}\left(\hat{\omega}_{1}+\hat{\omega}_{2}\right)$ & $+2\left(1+\frac{\hat{\omega}_{1}}{\hat{\omega}_{2}}\right) C_{1,2}^{(t)}\left(\hat{\omega}_{1}+\hat{\omega}_{2}\right)$ \\
\hline \multirow[t]{3}{*}{$b_{43,44}^{(t)}$} & $b_{43,44}^{(t)}\left(\hat{\omega}_{1}, 0\right)$ & $-2\left(\hat{\omega}_{1}+\hat{\omega}_{2}\right) C_{3,4}^{(t)^{\prime}}\left(\hat{\omega}_{1}+\hat{\omega}_{2}\right)$ & $b_{43,44}^{(t)}\left(\hat{\omega}_{1}, \hat{\omega}_{2}\right)=-2\left(\hat{\omega}_{1}+\hat{\omega}_{2}\right) C_{3,4}^{(t)^{\prime}}\left(\hat{\omega}_{1}+\hat{\omega}_{2}\right)$ \\
\hline & & $+2\left(1+\frac{\hat{\omega}_{1}}{\hat{\omega}_{2}}\right) C_{3,4}^{(t)}\left(\hat{\omega}_{1}+\hat{\omega}_{2}\right)$ & $+2\left(1+\frac{\hat{\omega}_{1}}{\hat{\omega}_{2}}\right) C_{3,4}^{(t)}\left(\hat{\omega}_{1}+\hat{\omega}_{2}\right)$ \\
\hline & & $\pm b_{34,35}\left(\hat{\omega}_{1}, \hat{\omega}_{2}\right)$ & $\pm b_{34,35}\left(\hat{\omega}_{1}, \hat{\omega}_{2}\right)$ \\
\hline
\end{tabular}

TABLE III: Summary of RPI constraints on the coefficients of the tensor currents in Eq. (93). The first column shows the constraints from type-I RPI on $b_{i}(\omega, 0)$, the second column shows the constraint on $b_{i}\left(\omega_{1}, \omega_{2}\right)$ from type-II RPI and the third column gives the combined constraint. Each \pm refers to the first and second terms in their row respectively. The final currents are displayed in Eqs. (9799), and are defined so that they automatically satisfy these constraints. 
There are 14 independent $O(\lambda)$ two-body operators given explicitly by

$$
\begin{aligned}
K_{1-4}^{(t)}(\omega) & =-\left(\bar{\xi}_{n} \frac{\not h}{2} i \overleftarrow{D}_{c}^{\perp} W\right)_{\omega} \frac{1}{\overline{\mathcal{P}}^{\dagger}} \Gamma_{1-4}^{\mu \nu} h_{v} \\
K_{5,6}^{(t)}(\omega) & =\left(\bar{\xi}_{n} i \overleftarrow{D}_{c \perp}^{\alpha} W\right)_{\omega} \frac{1}{\overline{\mathcal{P}}^{\dagger}}\left\{g_{\alpha[\mu}, \gamma_{\nu]}+v_{\alpha} \Gamma_{3}^{\mu \nu}, g_{\alpha[\mu}, v_{\nu]}-v_{\alpha} \Gamma_{4}^{\mu \nu}\right\} h_{v} \\
K_{7-10}^{(t)}(\omega) & =\frac{1}{m_{b}}\left(v \cdot \mathcal{P}_{\perp}\right)\left(\bar{\xi}_{n} W\right)_{\omega} \Gamma_{1-4}^{\mu \nu} h_{v} \\
K_{11-14}^{(t)}(\omega) & =\left(\bar{\xi}_{n} \frac{1}{\bar{n} \cdot i D_{c}} i v \cdot \vec{D}_{c}^{\perp} W\right)_{\omega} \frac{1}{n \cdot v} \Gamma_{1-4}^{\mu \nu} h_{v} .
\end{aligned}
$$

Their coefficients are fixed by reparameterization invariance in terms of the $\mathcal{O}\left(\lambda^{0}\right)$ Wilson coefficients $C_{1-4}^{(t)}(\omega)$ as

$$
\begin{array}{rlrl}
B_{1-4}^{(t)}(\omega) & =C_{1-4}^{(t)}(\omega), & B_{5}^{(t)}(\omega)=2 C_{3}^{(t)}(\omega), & B_{6}^{(t)}(\omega)=-2 C_{4}^{(t)}(\omega), \\
B_{7-10}^{(t)}(\omega) & =-2 C_{1-4}^{(t) \prime}(\omega), \quad B_{11-14}^{(t)}(\omega)=-2 C_{1-4}^{(t)}(\omega) .
\end{array}
$$

In addition, there are 7 three-body collinear operators given by

$$
\begin{aligned}
K_{15-18}^{(t)}\left(\omega_{1}, \omega_{2}\right) & =\frac{1}{m_{b}}\left(\bar{\xi}_{n} W\right)_{\omega_{1}} \Gamma_{1-4}^{\mu \nu}\left(\frac{1}{\overline{\mathcal{P}}} W^{\dagger} i g \not B_{c, \perp} W\right)_{\omega_{2}} h_{v} \\
K_{19}^{(t)}\left(\omega_{1}, \omega_{2}\right) & =\frac{1}{m_{b}}\left(\bar{\xi}_{n} W\right)_{\omega_{1}}\left(g_{\alpha[\mu}, \gamma_{\nu]}+v_{\alpha} \Gamma_{3}^{\mu \nu}\right)\left(\frac{1}{\overline{\mathcal{P}}} W^{\dagger} i g B_{c, \perp}^{\alpha} W\right)_{\omega_{2}} h_{v} \\
K_{20}^{(t)}\left(\omega_{1}, \omega_{2}\right) & =\frac{1}{m_{b}}\left(\bar{\xi}_{n} W\right)_{\omega_{1}}\left(g_{\alpha[\mu}, v_{\nu]}-v_{\alpha} \Gamma_{4}^{\mu \nu}\right)\left(\frac{1}{\overline{\mathcal{P}}} W^{\dagger} i g B_{c, \perp}^{\alpha} W\right)_{\omega_{2}} h_{v} \\
K_{21}^{(t)}\left(\omega_{1}, \omega_{2}\right) & =\frac{1}{m_{b}}\left(\bar{\xi}_{n} W\right)_{\omega_{1}} g_{\alpha[\mu}, n_{\nu]} \frac{1}{n \cdot v}\left(\frac{1}{\overline{\mathcal{P}}} W^{\dagger} i g B_{c, \perp}^{\alpha} W\right)_{\omega_{2}} h_{v} .
\end{aligned}
$$

Their coefficients $B_{15-21}^{(t)}\left(\hat{\omega}_{1}, \hat{\omega}_{2}\right)$ are not constrained by any symmetry of the effective theory and have to be determined by an explicit matching calculation.

At tree-level one matches onto the currents $K_{111}^{(t)}$ and the two-body limit of $K_{17}^{(t)}$ (using the analog of Eq. (73) ), and we agree with Ref. [13] on the form of these currents and the RPI constraint between $K_{1}^{(t)}$ and $K_{11}^{(t)}$. The remaining operators in Eqs. (97) and (99) are new and only appear beyond tree level (including the three body structure of $K_{11}^{(t)}$ ).

\section{SUMMARY FOR COEFFICIENTS, OPERATORS, AND FEYNMAN RULES}

In this section we summarize results that should be useful for future phenomelogical applications. In Section $\mathrm{VA}$ we summarize the full set of known matching results and compare with the literature, in section $\mathrm{VB}$ we give simplified expressions for our basis of currents in the frame $v_{\perp}=0, n \cdot v=1$, and in section $\nabla \mathrm{C}$ we give Feynman rules for the subleading currents and $\mathcal{L}_{\xi q}^{(1,2)}$. 


\section{A. Matching results for the currents}

In this subsection we summarize the one-loop matching results for the LO and NLO $J_{h l}$ Wilson coefficients. With $\hat{\omega}_{i}=\omega / m_{b}$ these coefficients are defined in previous sections as $C_{i}^{(d)}\left(\hat{\omega}, \mu / m_{b}\right)$ and $B_{i}^{(d)}\left(\hat{\omega}_{i}, \mu / m_{b}\right)$ respectively, where $(d)$ denotes whether the current is a scalar, pseudoscalar, vector, axial-vector, or a tensor.

For the LO currents the basis we use is different (though equivalent) to the basis used in Ref. 2]. Since the one-loop matching for the LO coefficients can be found in Ref. [2] it is useful to have the explicit relation between our basis of coefficients $C_{i}^{(d)}$ and the coefficients $C_{j}(j=1-12)$ that can be found there. We find

$$
\begin{array}{llll}
C_{0}^{(s)}=C_{1}, & C_{1}^{(v)}=C_{3}, & C_{1}^{(a)}=C_{6}, & C_{1}^{(t)}=C_{10}, \\
C_{0}^{(p)}=C_{2}, & C_{2}^{(v)}=C_{5}, & C_{2}^{(a)}=C_{8}, & C_{2}^{(t)}=-C_{12}, \\
& C_{3}^{(v)}=C_{4}-C_{3}, & C_{3}^{(a)}=C_{7}-C_{6}, & C_{3}^{(t)}=C_{10}-C_{9}, \\
& & & C_{4}^{(t)}=C_{12}+C_{10}-C_{11} .
\end{array}
$$

At tree level the matching between QCD and SCET is scheme independent. Matching with the full QCD currents $\bar{u}\left\{1, \gamma_{5}, \gamma_{\mu}, \gamma_{\mu} \gamma_{5}, i \sigma_{\mu \nu}\right\} b$ we find

$$
\begin{aligned}
& C_{0}^{(s)}=C_{0}^{(p)}=C_{1}^{(v)}=C_{1}^{(a)}=C_{1}^{(t)}=1, \\
& C_{2}^{(v)}=C_{3}^{(v)}=C_{2}^{(a)}=C_{3}^{(a)}=C_{2}^{(t)}=C_{3}^{(t)}=C_{4}^{(t)}=0 .
\end{aligned}
$$

At one-loop we use the $\overline{\mathrm{MS}}$ scheme with naive dimensional regularization (NDR) and match at $\mu=m_{b}$ to determine the $C_{i}^{(d)}\left(\hat{\omega}, \mu / m_{b}\right)$. Using Eq. (100) and results in Ref. [2] one finds

$$
\begin{aligned}
C_{0}^{(s, p)}(\hat{\omega}, 1) & =1-\frac{\alpha_{s}\left(m_{b}\right) C_{F}}{4 \pi}\left\{2 \ln ^{2}(\hat{\omega})+2 \operatorname{Li}_{2}(1-\hat{\omega})-\frac{2 \ln (\hat{\omega})}{1-\hat{\omega}}+\frac{\pi^{2}}{12}\right\}, \\
C_{1}^{(v, a)}(\hat{\omega}, 1) & =1-\frac{\alpha_{s}\left(m_{b}\right) C_{F}}{4 \pi}\left\{2 \ln ^{2}(\hat{\omega})+2 \operatorname{Li}_{2}(1-\hat{\omega})+\ln (\hat{\omega})\left(\frac{3 \hat{\omega}-2}{1-\hat{\omega}}\right)+\frac{\pi^{2}}{12}+6\right\}, \\
C_{1}^{(t)}(\hat{\omega}, 1) & =1-\frac{\alpha_{s}\left(m_{b}\right) C_{F}}{4 \pi}\left\{2 \ln ^{2}(\hat{\omega})+2 \operatorname{Li}_{2}(1-\hat{\omega})+\ln (\hat{\omega})\left(\frac{4 \hat{\omega}-2}{1-\hat{\omega}}\right)+\frac{\pi^{2}}{12}+6\right\}, \\
C_{2}^{(v, a)}(\hat{\omega}, 1) & =\frac{\alpha_{s}\left(m_{b}\right) C_{F}}{4 \pi}\left\{\frac{2}{(1-\hat{\omega})}+\frac{2 \hat{\omega} \ln (\hat{\omega})}{(1-\hat{\omega})^{2}}\right\}, \\
C_{2}^{(t)}(\hat{\omega}, 1) & =0, \\
C_{3}^{(v, a)}(\hat{\omega}, 1) & =\frac{\alpha_{s}\left(m_{b}\right) C_{F}}{4 \pi}\left\{\frac{(1-2 \hat{\omega}) \hat{\omega} \ln (\hat{\omega})}{(1-\hat{\omega})^{2}}-\frac{\hat{\omega}}{1-\hat{\omega}}\right\}, \\
C_{3}^{(t)}(\hat{\omega}, 1) & =\frac{\alpha_{s}\left(m_{b}\right) C_{F}}{4 \pi}\left\{\frac{-2 \hat{\omega} \ln (\hat{\omega})}{1-\hat{\omega}}\right\}, \\
C_{4}^{(t)}(\hat{\omega}, 1) & =0,
\end{aligned}
$$

where $C_{F}=4 / 3$ for color $S U(3)$. To determine the coefficients for scales $m_{b} \Lambda_{\mathrm{QCD}}<\mu^{2}<m_{b}^{2}$ we require their anomalous dimensions. ${ }^{9}$ The LO and NLO anomalous dimensions are

\footnotetext{
${ }^{9}$ The full NLO result requires a two-loop anomalous dimension which uses information from Ref. [35])
} 
universal and the running of these coefficients is given in Ref. [2] (or for the case $\hat{\omega}=1$ in Ref. [1]).

At NLO in $\lambda$ tree level matching of the QCD current $\bar{u} \Gamma b$ onto SCET gives [10, 12, 13]

$$
\begin{aligned}
J_{\text {tree }}^{(1 a)} & =-\bar{\xi}_{n, p^{\prime}} \frac{\not h}{2} i \overleftarrow{D D}_{c}^{\perp} W \frac{1}{\overline{\mathcal{P}}^{\dagger}} \Gamma h_{v}, \\
J_{\text {tree }}^{(1 b)} & =-\bar{\xi}_{n, p^{\prime}} \Gamma \frac{\not h}{2} \overrightarrow{\not D}_{c}^{\perp} W \frac{1}{n \cdot v m_{b}} h_{v}, \\
J_{\text {tree }}^{(1 c)} & =-\frac{2}{n \cdot v} \bar{\xi}_{n, p^{\prime}} \Gamma \frac{1}{i \bar{n} \cdot \vec{D}_{c}} i v \cdot \vec{D}_{c}^{\perp} W h_{v} .
\end{aligned}
$$

The normalization in Eq. (103) was first derived for $J^{(1 a)}$ in Ref. [10], and for $J^{(1 b, 1 c)}$ in Ref. 13. . Comparing Eq. (103) with our basis of currents we see that for any choice of $\Gamma$ the $J_{\text {tree }}^{(1 a, 1 c)}$ match onto a subset of our two-body currents. On the other hand $J_{\text {tree }}^{(1 b)}$ does not appear in the basis of two-body currents. Instead it is obtained from the projection of a subset of the three-body currents for cases where the corresponding coefficients $B_{i}\left(\hat{\omega}_{2}, \hat{\omega}_{2}\right)$ depend only on the sum $\hat{\omega}_{1}+\hat{\omega}_{2}$. This is certainly the case at tree level since the coefficients are $\hat{\omega}_{i}$ independent. The three-body structure of the currents can only show up at the level of one-loop matching.

Using Eq. (103) to determine the tree-level value of the NLO Wilson coefficients of the operators in Eqs. (7181, 86, 89, 97, 99) we find

$$
\begin{array}{llllrl}
B_{1}^{(s, p)}=1, & B_{1}^{(v, a)}=1, & B_{9,10}^{(v, a)}=0, & B_{1}^{(t)}=1, & B_{12-14}^{(t)}=0, \\
B_{2}^{(s, p)}=0, & B_{2-4}^{(v, a)}=0, & B_{11,12}^{(v, a)}=0, & B_{2-6}^{(t)}=0, & B_{15,16}^{(t)}=0, \\
B_{3}^{(s, p)}=-2, & B_{5-7}^{(v, a)}=0, & B_{13}^{(v, a)}=-1, & B_{7-10}^{(t)}=0, & B_{17}^{(t)}=1, \\
B_{4}^{(s, p)}=0, & B_{8}^{(v, a)}=-2, & B_{14}^{(v, a)}=0, & B_{11}^{(t)}=-2, & B_{18-21}^{(t)}=0 .
\end{array}
$$

These results are in agreement with the RPI constraints in Eqs. (72, 82, 87, 90, 98). Coefficients in Eq. (104) that are zero indicate that the corresponding currents can not be inferred at tree level since they are first matched onto at one-loop (or beyond). The full one-loop matching for all the $\mathcal{O}(\lambda)$ currents is not currently known from direct computations. However, many of the NLO coefficients are fixed in terms of the LO coefficients by RPI, namely $B_{0-3}^{(s, p)}(\hat{\omega}), B_{1-10}^{(v, a)}(\hat{\omega})$, and $B_{1-14}^{(t)}(\hat{\omega})$. Summarizing Eqs. (7282879098) we have

$$
\begin{aligned}
& B_{1}^{(s, p)}=C_{0}^{(s, p)}, \quad B_{2}^{(s, p)}=-2 C_{0}^{(s, p) \prime}, \quad B_{3}^{(s, p)}=-2 C_{0}^{(s, p)}, \\
& B_{1-3}^{(v, a)}=C_{1-3}^{(v, a)}, \quad B_{4}^{(v, a)}=-2 C_{3}^{(v, a)}, \quad B_{5-7}^{(v, a)}=-2 C_{1-3}^{(v, a) \prime}, \quad B_{8-10}^{(v, a)}=-2 C_{1-3}^{(v, a)}, \\
& B_{1-4}^{(t)}=C_{1-4}^{(t)}, \quad B_{5}^{(t)}=2 C_{3}^{(t)}, \quad B_{6}^{(t)}=-2 C_{4}^{(t)}, \quad B_{7-10}^{(t)}=-2 C_{1-4}^{(t) \prime} \text {, } \\
& B_{11-14}^{(t)}=-2 C_{1-4}^{(t)} \text {, }
\end{aligned}
$$

where to save space the $\hat{\omega}$ and $\mu$ dependence of the expressions on both sides of these equalities is suppressed. These results can be used to determine the matching for these coefficients at $\mu=m_{b}$ using Eq. (102). They also imply that the anomalous dimensions of these coefficients are determined by the anomalous dimension of the leading order coefficients [2], so their values for scales $m_{b} \Lambda_{\mathrm{QCD}}<\mu^{2}<m_{b}^{2}$ is known. 
For the coefficients of the 3-body operators, $B_{4}^{(s, p)}\left(\hat{\omega}_{1}, \hat{\omega}_{2}\right), \quad B_{11-14}^{(v, a)}\left(\hat{\omega}_{1}, \hat{\omega}_{2}\right)$, and $B_{15-21}^{(t)}\left(\hat{\omega}_{1}, \hat{\omega}_{2}\right)$, neither the one-loop matching results, nor even the LO anomalous dimensions are currently known.

Finally, we note that it is possible to relate the pseudoscalar and axial-vector coefficients from the scalar and vector coefficients. For massless quarks the QCD diagrams and SCET diagrams change in a trivial way under the chiral transformation, $q \rightarrow \gamma_{5} q$, and $\xi_{n} \rightarrow \gamma_{5} \xi_{n}$, provided we work in a scheme such as NDR. Therefore in this scheme the Wilson coefficients of operators with and without $\gamma_{5}$ are related (see for example Ref. [2] for the relations between LO coefficients). In other renormalization schemes these coefficients may differ.

\section{B. Summary of $\mathcal{O}(\lambda)$ currents in the frame $v_{\perp}=0, n \cdot v=1$}

In sections IVA through IVD we have derived the most general basis of heavy-to-light current to $\mathcal{O}(\lambda)$ in an arbitrary frame. However, for applications it is often most convenient to pick a frame where $v_{\perp}=0$ and $v \cdot n=1$. In this frame the currents $K_{2,3}^{(s, p)}, K_{5-10}^{(v, a)}$, and $K_{7-14}^{(t)}$ drop out. Thus there are only $(2,2,8,8,13)$ order $\mathcal{O}(\lambda)$ heavy-to-light currents which are (scalar, pseudo-scalar, vector, axial-vector, tensor). In this section we summarize our results with this choice of basis vectors.

In this frame our leading order results for the $J_{h l}$ currents with a complete set of Dirac structures can be summarized as

$$
\begin{aligned}
J^{(0)} & =\int d \omega C_{i}^{(d)}(\hat{\omega}) J_{i}^{(0)}(\omega), \\
J_{i}^{(0)}(\omega) & =\left(\bar{\xi}_{n} W\right)_{\omega} \Gamma_{i}^{(d)} h_{v},
\end{aligned}
$$

where in $\Gamma_{i}^{(d)}$ the $(d)$ specifies the type of current (scalar,vector,...), the $i$ specifies the member of the complete set of possible structures of that type, and the Wilson coefficients are $C_{i}^{(d)}(\hat{\omega})$. For the minimum basis of Dirac structures we found

$$
\begin{aligned}
\Gamma_{0}^{(s)} & =1, \quad \Gamma_{0}^{(p)}=\gamma_{5}, \\
\Gamma_{\{1,2,3\}}^{(v)} & =\left\{\gamma_{\mu}, v_{\mu}, n_{\mu}\right\} \\
\Gamma_{\{1,2,3\}}^{(a)} & =\left\{\gamma_{\mu} \gamma_{5}, v_{\mu} \gamma_{5}, n_{\mu} \gamma_{5}\right\} \\
\Gamma_{\{1,2,3,4\}}^{(t)} & =\left\{i \sigma_{\mu \nu}, \gamma_{[\mu}, v_{\nu]}, \gamma_{[\mu}, n_{\nu]}, n_{[\mu}, v_{\nu]}\right\},
\end{aligned}
$$

which is simply a linear combination of the basis in Ref. [2]. At order $\lambda$ our corresponding results for $J_{h l}$ in this frame can be summarized as

$$
\begin{aligned}
J^{(1 a)} & =\int d \omega B_{i}^{(d)}(\hat{\omega}) J_{i}^{(1 a)}(\omega) \\
J^{(1 b)} & =\int d \omega_{1} d \omega_{2} B_{i}^{(d)}\left(\hat{\omega}_{1}, \hat{\omega}_{2}\right) J_{i}^{(1 b)}\left(\omega_{1}, \omega_{2}\right) \\
J_{i}^{(1 a)}(\omega) & =\left(\bar{\xi}_{n} i \overleftarrow{D}_{c \alpha}^{\perp} W\right)_{\omega} \frac{1}{\overline{\mathcal{P}}^{\dagger}} \Upsilon_{i}^{(d) \alpha} h_{v} \\
J_{i}^{(1 b)}\left(\omega_{1}, \omega_{2}\right) & =\frac{1}{m_{b}}\left(\bar{\xi}_{n} W\right)_{\omega_{1}} \Theta_{i}^{(d) \alpha}\left(\frac{1}{\overline{\mathcal{P}}} W^{\dagger} i g B_{c \alpha}^{\perp} W\right)_{\omega_{2}} h_{v}
\end{aligned}
$$



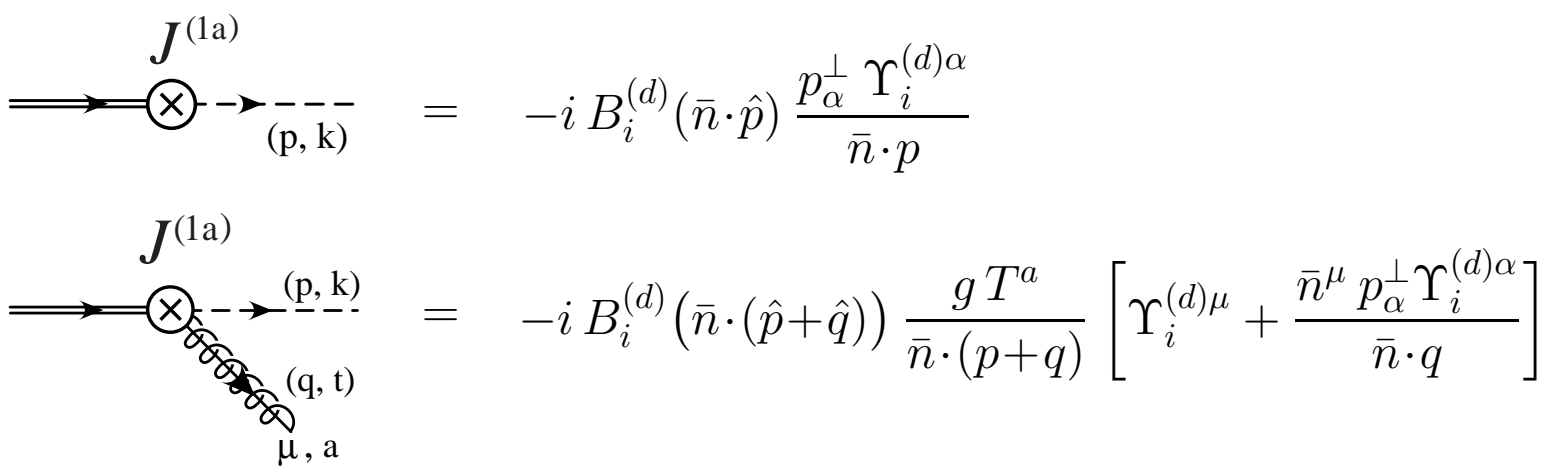

FIG. 1: Feynman rules for the $O(\lambda)$ currents $J^{(1 a)}$ in Eq. (108) with zero and one gluon (the fermion spinors are suppressed). For the collinear particles we show their (label,residual) momenta, where label momenta are $p, q \sim \lambda^{0,1}$ and residual momenta are $k, t \sim \lambda^{2}$. Momenta with a hat are normalized to $m_{b}, \hat{p}=p / m_{b}$ etc.

where

$$
\begin{aligned}
\Upsilon_{1}^{(s) \alpha} & =\gamma_{\perp}^{\alpha} \frac{\not h}{2}, \quad \Theta_{4}^{(s) \alpha}=\gamma_{\perp}^{\alpha}, \quad \Upsilon_{1}^{(p) \alpha}=\gamma_{\perp}^{\alpha} \frac{\not h}{2} \gamma_{5}, \quad \Theta_{4}^{(p) \alpha}=\gamma_{5} \gamma_{\perp}^{\alpha}, \\
\Upsilon_{1-4}^{(v) \alpha} & =\left\{\gamma_{\perp}^{\alpha} \frac{\not h}{2} \gamma^{\mu}, \gamma_{\perp}^{\alpha} \frac{\not h}{2} v^{\mu}, \gamma_{\perp}^{\alpha} \frac{\not h}{2} n^{\mu}, g_{\perp}^{\alpha \mu}\right\}, \quad \Theta_{11-14}^{(v) \alpha}=\left\{\gamma^{\mu} \gamma_{\perp}^{\alpha}, v^{\mu} \gamma_{\perp}^{\alpha}, n^{\mu} \gamma_{\perp}^{\alpha}, g_{\perp}^{\mu \alpha}\right\}, \\
\Upsilon_{1-4}^{(a) \alpha} & =\left\{\gamma_{\perp}^{\alpha} \frac{\not h}{2} \gamma^{\mu}, \gamma_{\perp}^{\alpha} \frac{\not h}{2} v^{\mu}, \gamma_{\perp}^{\alpha} \frac{\not h}{2} n^{\mu}, g_{\perp}^{\alpha \mu}\right\} \gamma_{5}, \quad \Theta_{11-14}^{(a) \alpha}=\left\{\gamma^{\mu} \gamma_{5} \gamma_{\perp}^{\alpha}, v^{\mu} \gamma_{5} \gamma_{\perp}^{\alpha}, n^{\mu} \gamma_{5} \gamma_{\perp}^{\alpha},, g_{\perp}^{\mu \alpha} \gamma_{5}\right\}, \\
\Upsilon_{1-6}^{(t) \alpha} & =\left\{i \gamma_{\perp}^{\alpha} \frac{\not h}{2} \sigma^{\mu \nu}, \gamma_{\perp}^{\alpha} \frac{\not h}{2} \gamma^{[\mu,} v^{\nu]}, \gamma_{\perp}^{\alpha} \frac{\not h}{2} \gamma^{[\mu,} n^{\nu]}, \gamma_{\perp}^{\alpha} n^{[\mu,} v^{\nu]}, g_{\perp}^{\alpha[\mu,} \gamma^{\nu]}, g_{\perp}^{\alpha[\mu,} v^{\nu]}\right\}, \\
\Theta_{15-21}^{(t) \alpha} & =\left\{i \sigma^{\mu \nu} \gamma_{\perp}^{\alpha}, \gamma^{[\mu,} v^{\nu]} \gamma_{\perp}^{\alpha}, \gamma^{[\mu,} n^{\nu]} \gamma_{\perp}^{\alpha}, n^{[\mu,} v^{\nu]} \gamma_{\perp}^{\alpha}, g_{\perp}^{\alpha[\mu,} \gamma^{\nu]}, g_{\perp}^{\alpha[\mu,} v^{\nu]}, g_{\perp}^{\alpha[\mu,} n^{\nu]}\right\} .
\end{aligned}
$$

Note that due to Eq. (14) the form of $J^{(1 b)}$ in Eq. (108) is identical to the form of the currents that was used in Ref. [15] since $\left[W^{\dagger} i \vec{D}_{c \alpha}^{\perp} W\right]=\left[1 / \overline{\mathcal{P}} W^{\dagger} i g B_{c \alpha}^{\perp} W\right]$.

\section{Feynman rules for $J_{h l}$ and $\mathcal{L}_{\xi q}$}

In this subsection Feynman rules are given for the $\mathcal{O}(\lambda)$ heavy-to-light currents $J^{(1 a)}$ and $J^{(1 b)}$ in Eq. (108) which are valid in a frame where $v_{\perp}=0$ and $v \cdot n=1$. We also give the Feynman rules that follow from the final form of the $\mathcal{L}_{\xi q}^{(1,2 a, 2 b)}$ Lagrangians in Eq. (42).

For the subleading currents the zero and one gluon Feynman rules for $J^{(1 a)}$ and $J^{(1 b)}$ are shown in Figs. 1 and 2 respectively. (From the results in the previous sections the Feynman rules for the currents with $v_{\perp} \neq 0$ and $v \cdot n \neq 1$ can also be easily derived.) For $J^{(1 a)}$ the Wilson coefficients depend only on the total $\lambda^{0}$ collinear momentum, while for $J^{(1 a)}$ the coefficients depend on how the momentum is divided between the quark and gluons. The $J^{(1 a)}$ current has non-vanishing Feynman rules with zero or one $A_{n}^{\perp}$ gluon and any number of $\bar{n} \cdot A_{n}$ gluons. The possible gluons that appear in the $J^{(1 b)}$ currents are similar, but the current vanishes unless it has one or more collinear gluons present. 

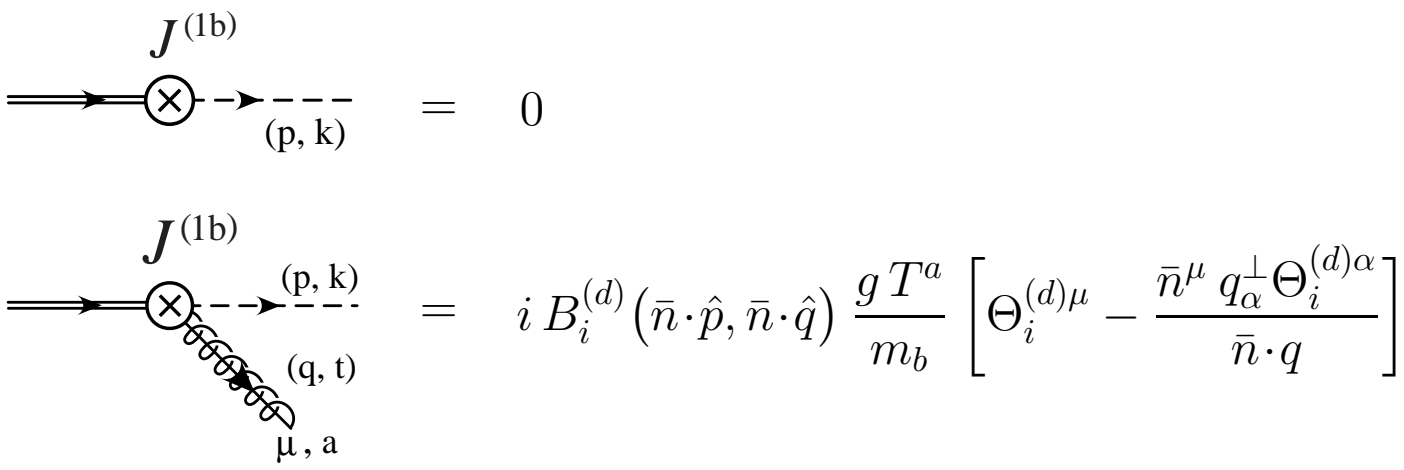

FIG. 2: Feynman rules for the $O(\lambda)$ currents $J^{(1 b)}$ in Eq. (108) with zero and one gluon. For the collinear particles we show their (label,residual) momenta, where label momenta are $p, q, q_{i} \sim \lambda^{0,1}$ and residual momenta are $k, t \sim \lambda^{2}$. Momenta with a hat are normalized to $m_{b}, \hat{p}=p / m_{b}$ etc.

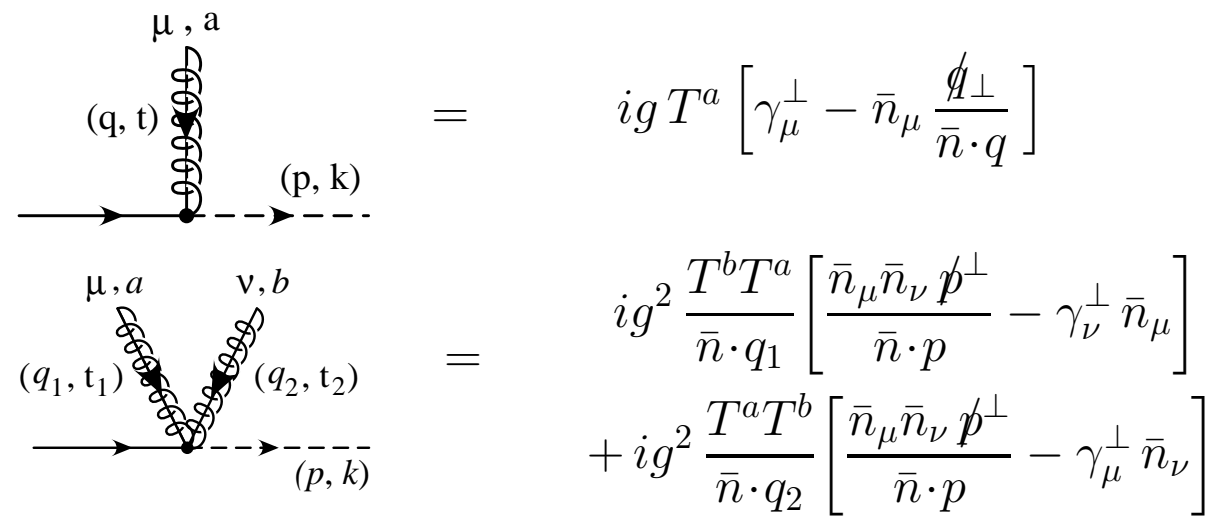

FIG. 3: Feynman rules for the subleading usoft-collinear Lagrangian $\mathcal{L}_{\xi q}^{(1)}$ with one and two collinear gluons (springs with lines through them). The solid lines are usoft quarks while dashed lines are collinear quarks. For the collinear particles we show their (label,residual) momenta. (The fermion spinors are suppressed.)

For the mixed usoft-collinear Lagrangians from Eq. (42),

$$
\begin{aligned}
\mathcal{L}_{\xi q}^{(1)} & =\bar{\xi}_{n} \frac{1}{i \bar{n} \cdot D_{c}} i g \not \mathbb{P}_{c}^{\perp} W q_{u s}+\text { h.c. }, \\
\mathcal{L}_{\xi q}^{(2 a)} & =\bar{\xi}_{n} \frac{1}{i \bar{n} \cdot D_{c}} i g M W q_{u s}+\text { h.c. }, \\
\mathcal{L}_{\xi q}^{(2 b)} & =\bar{\xi}_{n} \frac{\not h}{2} i \not D_{\perp}^{c} \frac{1}{\left(i \bar{n} \cdot D_{c}\right)^{2}} i g \not_{\perp}^{c} W q_{u s}+\text { h.c. },
\end{aligned}
$$

all Feynman rules involve at least one collinear gluon. From $\mathcal{L}_{\xi q}^{(1)}$ we obtain Feynman rules with zero or one $A_{n}^{\perp}$ gluons and any number of $\bar{n} \cdot A_{n}$ gluons. The one and two-gluon results are shown in Fig. 3 .

For $\mathcal{L}_{\xi q}^{(2 a)}$ we have Feynman rules with zero or one $\left\{n \cdot A_{n}, A_{u s}^{\perp}\right\}$ gluon and any number of $\bar{n} \cdot A_{n}$ gluons. The one and two-gluon results are shown in Fig. 4. Finally, for $\mathcal{L}_{\xi q}^{(2 b)}$ one finds Feynman rules with zero, one, or two $A_{n}^{\perp}$ gluons and any number of $\bar{n} \cdot A_{n}$ gluons. In this case the one and two gluon Feynman rules are shown in Fig. 5. Note that it is important to treat the contributions from $\mathcal{L}_{\xi q}^{(2 a)}$ and $\mathcal{L}_{\xi q}^{(2 b)}$ separately since they show up in different parts 


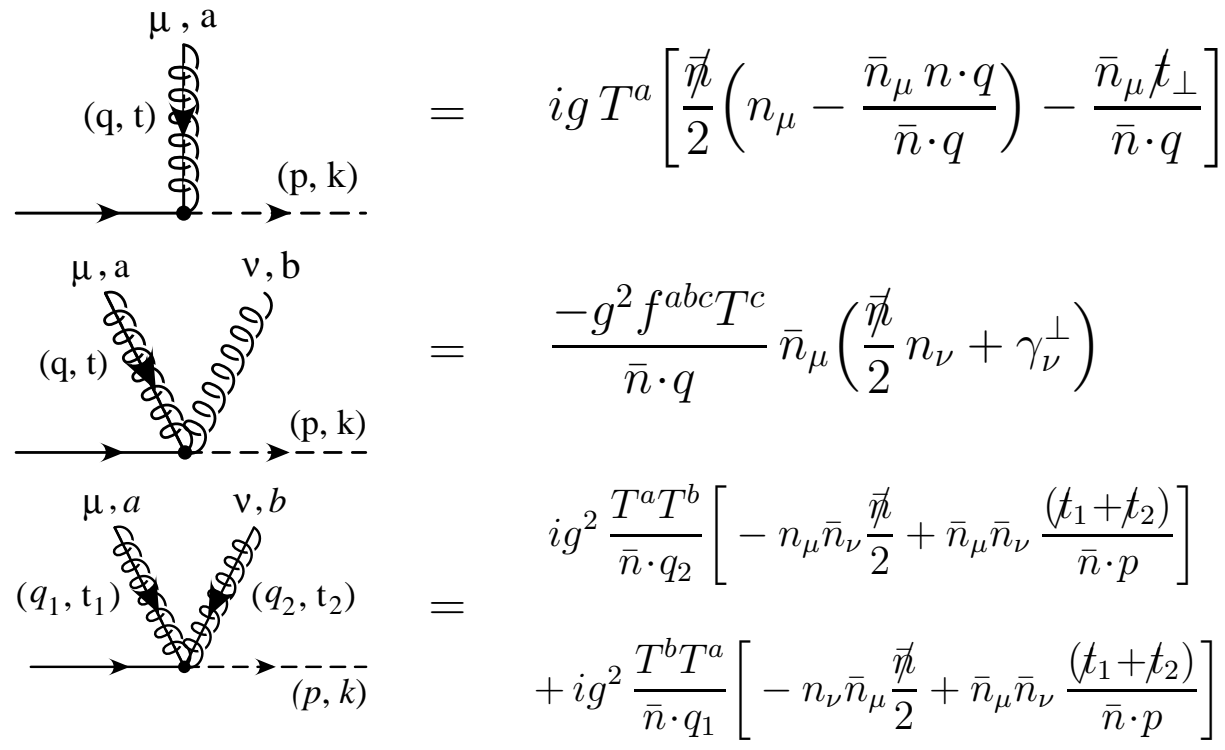

FIG. 4: Feynman rules for the $O\left(\lambda^{2}\right)$ usoft-collinear Lagrangian $\mathcal{L}_{\xi q}^{(2 a)}$ with one and two gluons. The spring without a line through it is an usoft gluon. For the collinear particles we show their (label,residual) momenta, where label momenta are $p, q, q_{i} \sim \lambda^{0,1}$ and residual momenta are $k, t, t_{i} \sim \lambda^{2}$.

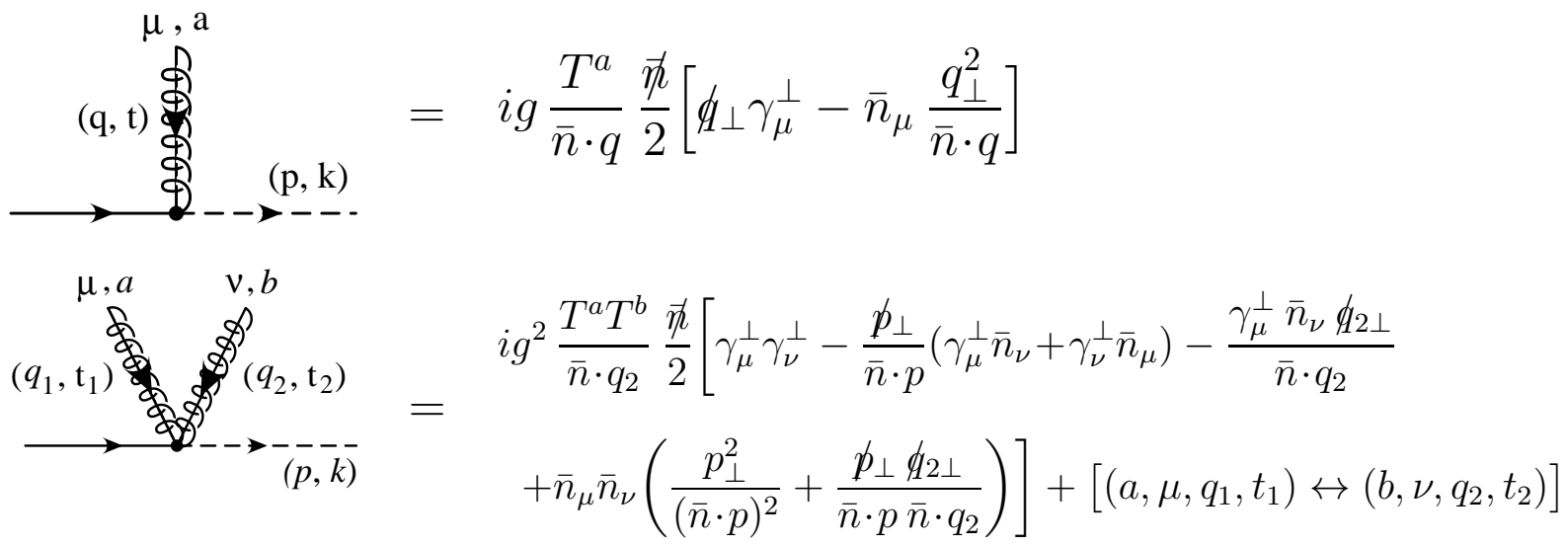

FIG. 5: Feynman rules for the $O\left(\lambda^{2}\right)$ usoft-collinear Lagrangian $\mathcal{L}_{\xi q}^{(2 b)}$ with one and two gluons. For the collinear particles we show their (label,residual) momenta, where label momenta are $p, q, q_{i} \sim$ $\lambda^{0,1}$ and residual momenta are $k, t, t_{i} \sim \lambda^{2}$.

of the heavy-to-light factorization formulae derived in Ref. [15] and shown in Eq. (11).

For $\mathcal{L}_{\xi q}^{(2 a)}+\mathcal{L}_{\xi q}^{(2 b)}$ the Feynman rules are different than one would derive using the intermediate form Eq. (30), since in transforming to the final form the equations of motion were applied. However, observable predictions that are consistently made with either set of Feynman rules will agree. 


\section{LEADING POWER PREDICTIONS FOR $B$ TO PSEUDOSCALAR MESONS.}

As a phenomenological example, we consider the form factors for $B \rightarrow \pi \ell \nu$, or more generally the form factors for $B \rightarrow P$ where $P$ is a pseudoscalar meson (calculations using the factorization theorem in Eq. (11) for the vector meson are just as straightforward). For pseudoscalars there are three form factors in QCD, which are conventionally defined by

$$
\begin{aligned}
\left\langle P(p)\left|\bar{q} \gamma^{\mu} b\right| \bar{B}\left(p_{b}\right)\right\rangle & =f_{+}\left(q^{2}\right)\left[p_{b}^{\mu}+p^{\mu}-\frac{m_{B}^{2}-m_{P}^{2}}{q^{2}} q^{\mu}\right]+f_{0}\left(q^{2}\right) \frac{m_{B}^{2}-m_{P}^{2}}{q^{2}} q^{\mu}, \\
\left\langle P(p)\left|\bar{q} i \sigma^{\mu \nu} q_{\nu} b\right| \bar{B}\left(p_{b}\right)\right\rangle & =-\frac{f_{T}\left(q^{2}\right)}{m_{B}+m_{P}}\left[q^{2}\left(p_{b}^{\mu}+p^{\mu}\right)-\left(m_{B}^{2}-m_{P}^{2}\right) q^{\mu}\right],
\end{aligned}
$$

where $q=p_{b}-p$.

For the region where $Q=\left\{E, m_{b}\right\} \gg \Lambda_{\mathrm{QCD}}$ (ie. small $q^{2}$ ) one can use large energy factorization to study the form factors. For pions our expansion parameter $\Lambda_{\mathrm{QCD}} / \bar{n} \cdot p \sim$ $0.5 \mathrm{GeV} /(2 E)$ becomes $1 / 4$ for $E \simeq 1 \mathrm{GeV}$. This makes the region of $q^{2}$ where the expansion is valid roughly $0 \lesssim q^{2} \lesssim 10 \mathrm{GeV}^{2}$. In SCET the form factors $f_{+}, f_{0}, f_{T}$ split themselves up into contributions associated with three momentum regions: Wilson coefficients for $p^{2} \sim Q^{2}$, two jet functions $J_{a, b}$ for $p^{2} \sim Q \Lambda_{\mathrm{QCD}}$, universal light-cone wavefunctions for $p^{2} \sim \Lambda_{\mathrm{QCD}}^{2}$, and a single non-factorizable form factor $\zeta_{P}$ (containing both $p^{2} \sim Q \Lambda_{\mathrm{QCD}}$ and $p^{2} \sim \Lambda_{\mathrm{QCD}}^{2}$ ). The leading contributions therefore split into factorizable $(\mathrm{F})$ and non-factorizable (NF) contributions. ${ }^{10}$ This decomposition was defined by the proof of a factorization formula for these form factors in Ref. 15.

$$
\begin{aligned}
f^{Q C D}\left(q^{2}\right)= & f^{\mathrm{F}}(Q)+f^{\mathrm{NF}}(Q)+\ldots \\
f^{F}(Q)= & N_{0} \int_{0}^{1} d z \int_{0}^{1} d x \int_{0}^{\infty} d r_{+} T\left(z, Q, \mu_{0}\right) \\
& \quad \times J\left(z, x, r_{+}, Q, \mu_{0}, \mu\right) \phi_{P}(x, \mu) \phi_{B}\left(r_{+}, \mu\right), \\
f^{\mathrm{NF}}(Q)= & C_{k}(Q, \mu) \zeta_{k}^{P}(Q, \mu) .
\end{aligned}
$$

where $f^{\mathrm{F}}(Q) \sim f^{\mathrm{NF}}(Q) \sim Q^{-3 / 2}$ and the ellipses denote terms that are suppressed by more powers of $1 / Q$. Here $\phi_{B}=\phi_{B}^{ \pm}$.

To separate the scales $Q^{2}$ and $Q \Lambda$ we match QCD onto an $\operatorname{SCET}_{\mathrm{I}}$. The scales $Q \Lambda$ and $\Lambda^{2}$ are then separated by matching $\mathrm{SCET}_{\mathrm{I}}$ onto an $\mathrm{SCET}_{\mathrm{II}}$ [15]. Operators in $\mathrm{SCET}_{\mathrm{I}}$ are divided into $\mathrm{F}$ and $\mathrm{NF}$ categories depending on the form of the result of factoring usoft gluons from collinear fields. In $\mathrm{SCET}_{\mathrm{I}}$ the $\mathrm{F}$ contributions are from the time-ordered products

$$
\begin{aligned}
& T_{1}^{F}=\int \mathrm{d}^{4} x T\left\{J^{(1 a)}(0), i \mathcal{L}_{\xi q}^{(1)}(x)\right\}, \quad T_{2}^{F}=\int \mathrm{d}^{4} x T\left\{J^{(1 b)}(0), i \mathcal{L}_{\xi q}^{(1)}(x)\right\} \\
& T_{3}^{F}=\int \mathrm{d}^{4} x T\left\{J^{(0)}(0), i \mathcal{L}_{\xi q}^{(2 b)}(x)\right\},
\end{aligned}
$$

\footnotetext{
${ }^{10}$ Here the phrase non-factorizable simply refers to the fact that the matrix elements can not be expressed in terms of convolutions with the standard light-cone wavefunctions.
} 
where the currents are taken from Eq. (108) and the usoft-collinear Lagrangians from Eq. (110). After factorization of usoft and collinear fields, the $T_{i}^{F}$ 's are matched onto softcollinear SCET $_{\mathrm{II}}$ operators. The collinear matrix elements are given in terms of jet functions $J\left(z, x, r_{+}, Q\right)$, and the soft operators are given in terms of $B$ light-cone wave functions $\phi_{ \pm}\left(r_{+}\right)$ defined as [19, 22]

$$
\begin{aligned}
& \left\langle 0\left|\bar{q}\left(x^{-}\right) S_{n}\left(x^{-}, 0\right) \Gamma h_{v}(0)\right| \bar{B}(v)\right\rangle \\
& \quad=-\frac{i}{2} f_{B} m_{B} \int \mathrm{d} r^{+} e^{-\frac{i}{2} r^{+} x^{-}} \operatorname{Tr}\left\{\frac{1+\psi}{2}\left[\frac{\not h \not h}{4} \phi_{B}^{+}\left(r_{+}\right)+\frac{\not h \not h}{4} \phi_{B}^{-}\left(r_{+}\right)\right] \gamma_{5} \Gamma\right\} .
\end{aligned}
$$

A few general properties of the factorizable term $f^{F}(Q)$ can be given without an explicit computation. First, the matrix elements of $T_{1,2}^{F}$ can only depend on $\phi_{B}^{+}\left(r_{+}\right)$. This follows from the explicit form of the subleading Lagrangian $\mathcal{L}_{\xi q}^{(1)}$, where $\bar{\xi}_{n}=\bar{\xi}_{n} \frac{n \vec{n}}{4}$, so the usoft field $q_{u s}$ appears only in the combination $\bar{q}_{u s} \frac{\eta \vec{p}}{4}$. Using (114) this implies that only the $\phi_{B}^{+}\left(r_{+}\right)$term gives a nonvanishing contribution. On the other hand, the factorizable operator $T_{3}^{F}$ depends on the combination $\bar{q}_{u s} \frac{\vec{h}}{2}$, so its matrix element can only contain $\phi_{B}^{-}\left(r_{+}\right)$. At tree-level the jet function $J$ from the matrix element of $T_{3}^{F}$ vanishes, but a nonzero result could appear at one-loop order. However, the matrix element of $T_{3}^{F}$ contains the leading order current $J^{(0)}$, so it obeys the same symmetry relations as those derived for the nonfactorizable part $f^{N F}(Q)$ [15]. Therefore, although this matrix element is factorizable it does not increase the number of unknown non-perturbative functions since for phenomenological analyses $\phi_{B}^{-}$can be absorbed in $\zeta_{k}^{M}$. With this choice, all remaining factorizable contributions are expressible in terms of just $\phi_{B}^{+}\left(r_{+}\right)$.

Using the approach explained in [15] we can obtain the results for the form factors. After factorization of usoft and collinear fields the T-products of collinear fields coming from $T_{1,2}^{F}$ are given by (using Eqs. (108) and (110) )

$$
\begin{aligned}
\mathcal{J}_{\omega}^{1 a}(x) & \equiv T\left[\bar{\xi}_{n} i \overleftarrow{D}_{c \alpha}^{\perp} W\right]_{\omega}^{i A}(0)\left[W^{\dagger} i g B_{\perp}^{c} W \frac{1}{\overline{\mathcal{P}}^{\dagger}} W^{\dagger} \xi_{n}\right]_{0}^{j B}(x) \\
\mathcal{J}_{\omega_{1}, \omega_{2}}^{1 b}(x) & \equiv T\left[\left[\bar{\xi}_{n} W\right]_{\omega_{1}}\left[\frac{1}{\overline{\mathcal{P}}} W^{\dagger} i g B_{\perp \alpha}^{c} W\right]_{\omega_{2}}\right]^{i A}(0)\left[W^{\dagger} i g \mathbb{B}_{\perp}^{c} W \frac{1}{\overline{\mathcal{P}}^{\dagger}} W^{\dagger} \xi_{n}\right]_{0}^{j B}(x),
\end{aligned}
$$

where $i, j$ are Dirac indices and here $A, B$ are color indices in the fundamental representation. The functions $\mathcal{J}_{1 a, 1 b}$ are collinear gauge invariant and satisfy the spin structure constraints $\not h \mathcal{J}_{1 a, 1 b}=\mathcal{J}_{1 a, 1 b} \not h=0$, and $\operatorname{tr}\left[\mathcal{J}_{1 a, 1 b}\right]=0$.

Taking into account constraints from the Dirac structure of the effective theory fields one can easily find the most general form of the operators appearing in the matching of $\mathcal{J}^{1 a, 1 b}$ onto operators in $\mathrm{SCET}_{\mathrm{II}}$. The jet functions $J_{a, b}$ are defined by the terms which contribute 
on a pseudoscalar state

$$
\begin{aligned}
& \mathcal{J}_{\omega}^{1 a}(x)=i \delta\left(x_{+}\right) \delta^{2}\left(x_{\perp}\right)\left[\gamma_{\perp}^{\alpha} \not h \gamma_{5}\right]^{j i} \delta^{A B} \frac{1}{\omega} \int d \bar{\eta} \int \frac{d r^{+}}{2 \pi} e^{-\frac{i}{2} r^{+} x^{-}} \\
& \times J_{a}\left(\bar{\eta}, r^{+}\right)\left[\left(\bar{\xi}_{n} W\right) \delta\left(\bar{\eta}-\overline{\mathcal{P}}_{+}\right) \frac{\not h}{2} \gamma_{5}\left(W^{\dagger} \xi_{n}\right)\right]_{\mathrm{II}}+\cdots \\
& \mathcal{J}_{\omega_{1}, \omega_{2}}^{1 b}(x)=i \delta\left(x_{+}\right) \delta^{2}\left(x_{\perp}\right)\left[\gamma_{\perp}^{\alpha} \not h \gamma_{5}\right]^{j i} \delta^{A B} \frac{1}{\omega_{1}+\omega_{2}} \int d \bar{\eta} \int \frac{d r^{+}}{2 \pi} e^{-\frac{i}{2} r^{+} x^{-}} \\
& \times J_{b}\left(\bar{\omega}, \bar{\eta}, r^{+}\right)\left[\left(\bar{\xi}_{n} W\right) \delta\left(\bar{\eta}-\overline{\mathcal{P}}_{+}\right) \frac{\not \not h}{2} \gamma_{5}\left(W^{\dagger} \xi_{n}\right)\right]_{\mathrm{II}}+\cdots
\end{aligned}
$$

where $\bar{\omega}=\omega_{1}-\omega_{2}$. We have suppressed the dependence of $J_{1 a, 1 b}$ on the $\mu$ 's and on $\omega, \omega_{1}+\omega_{2}$ (the latter combinations would be simply set to $\bar{n} \cdot p$ in the pseudoscalar matrix element by momentum conservation [3]). The ellipses in Eq. (116) denote color octet terms and other operators which do not contribute for a pseudoscalar meson $P$.

Using Eq. (116) the operators in $\left\langle P_{n}(p)\left|T_{1,2}^{F}\right| \bar{B}_{v}\right\rangle$ factor into a product of matrix elements that can be evaluated with Eq. (114) and Eq. $(12,13)$ of Ref. [7]. Switching variables to $x, z$ by using $\bar{\omega}=(2 x-1) \bar{n} \cdot p$ and $\bar{\eta}=(2 z-1) \bar{n} \cdot p$ we find the following factorization theorems which are valid at leading order ${ }^{11}$ in $1 / Q$ and all orders in $\alpha_{s}$

$$
\begin{aligned}
f_{+}\left(q^{2}\right)= & N_{0} \int_{0}^{1} \mathrm{~d} x \int_{0}^{\infty} \mathrm{d} r_{+}\left[\frac{2 E-m_{B}}{m_{B}} T_{a}^{(+)}\left(E, \mu_{0}\right) J_{a}\left(x, r_{+}, Q, \mu_{0}, \mu\right)\right. \\
& \left.+\frac{2 E}{m_{b}} \int_{0}^{1} \mathrm{~d} z T_{b}^{(+)}\left(E, z, \mu_{0}\right) J_{b}\left(z, x, r_{+}, Q, \mu_{0}, \mu\right)\right] \phi_{P}(x, \mu) \phi_{B}^{+}\left(r_{+}, \mu\right) \\
& +\left\{C_{1}^{(v)}\left(2 \hat{E}, \mu_{0}\right)+\frac{E}{m_{B}} C_{2}^{(v)}\left(2 \hat{E}, \mu_{0}\right)+C_{3}^{(v)}\left(2 \hat{E}, \mu_{0}\right)\right\} \zeta^{P}\left(Q, \mu_{0}\right), \\
f_{0}\left(q^{2}\right)= & N_{0} \int_{0}^{1} \mathrm{~d} x \int_{0}^{\infty} \mathrm{d} r_{+}\left[\frac{2 E\left(m_{B}-2 E\right)}{m_{B}^{2}} T_{a}^{(0)}\left(E, \mu_{0}\right) J_{a}\left(x, r_{+}, Q, \mu_{0}, \mu\right)\right. \\
& \left.+\frac{4 E^{2}}{m_{b} m_{B}} \int_{0}^{1} \mathrm{~d} z T_{b}^{(0)}\left(E, z, \mu_{0}\right) J_{b}\left(z, x, r_{+}, Q, \mu_{0}, \mu\right)\right] \phi_{P}(x, \mu) \phi_{B}^{+}\left(r_{+}, \mu\right) \\
& +\frac{2 E}{m_{B}}\left\{C_{1}^{(v)}\left(2 \hat{E}, \mu_{0}\right)+\frac{m_{B}-E}{m_{B}} C_{2}^{(v)}\left(2 \hat{E}, \mu_{0}\right)+C_{3}^{(v)}\left(2 \hat{E}, \mu_{0}\right)\right\} \zeta^{P}\left(E, \mu_{0}\right), \\
m_{B}+m_{P} & N_{0}^{1} \mathrm{~d} x \int_{0}^{\infty} \mathrm{d} r_{+}\left[-T_{a}^{(T)}\left(E, \mu_{0}\right) J_{a}\left(x, r_{+}, Q, \mu_{0}, \mu\right)\right. \\
& \left.-\frac{2 E}{m_{b}} \int_{0}^{1} \mathrm{~d} z T_{b}^{(T)}\left(E, z, \mu_{0}\right) J_{b}\left(z, x, r_{+}, Q, \mu_{0}, \mu\right)\right] \phi_{P}(x, \mu) \phi_{B}^{+}\left(r_{+}, \mu\right) \\
& +\frac{m_{B}+m_{P}}{m_{B}}\left\{C_{1}^{(t)}\left(2 \hat{E}, \mu_{0}\right)-C_{2}^{(t)}\left(2 \hat{E}, \mu_{0}\right)-C_{4}^{(t)}\left(2 \hat{E}, \mu_{0}\right)\right\} \zeta^{P}\left(E, \mu_{0}\right),
\end{aligned}
$$

where $\hat{E}=E / m_{b}, Q=\left\{E, m_{b}\right\}$, and the normalization coefficient is given by $N_{0}=$ $f_{B} f_{P} m_{B} /\left(4 E^{2}\right)$. The matrix element involving non-factorizable operators gives $\zeta^{P}(Q, \mu)$

${ }^{11}$ We kept a kinematic factor of $m_{P}$ in the prefactor of $f_{T}$ even though it is formally power suppressed. 
which is the reduced form factor describing decays to a pseudoscalar meson $P$. The quantities in square brackets and curly brackets are calculable, that is the $T_{a, b}$ 's and $J_{a, b}$ 's have expansions in $\alpha_{s}(Q)$ and $\alpha_{s}(\sqrt{Q \Lambda})$ respectively. Note that the $J_{a, b}$ are universal, meaning that at any order in $\alpha_{s}$ it is these same jet functions which appear for any pseudoscalar meson and independent of which form factor $f_{+, 0, T}$ we consider. Therefore, the factorization theorem still gives information even in the case where we assume that $\alpha_{s}(\sqrt{Q \Lambda})$ is non-perturbative.

Working at $\mathcal{O}\left(\alpha_{s}\left(\mu_{0}\right)\right)$ (ie. tree level) for the jet functions gives

$$
\begin{aligned}
J_{1 a}\left(x, r^{+}\right) & =\frac{\pi C_{F}}{N_{c}} \frac{\alpha_{s}\left(\mu_{0}\right)}{x r^{+}} \\
J_{1 b}\left(z, x, r^{+}\right) & =\frac{\pi C_{F}}{N_{c}} \frac{\alpha_{s}\left(\mu_{0}\right)}{x r^{+}} \delta(z-x) .
\end{aligned}
$$

At this level the $z$ integrals in Eq. (118) disappear because the tree level jet gives a $\delta(z-x)$, and this causes the $z$ variable in the $T_{b}$ 's to be replaced by $x$. The $T_{a, b}^{(j)}$ are combinations of Wilson coefficients appearing in the $J^{(1 a, 1 b)}$ currents given in Eq. (108) and should be evaluated at a scale $\mu_{0}^{2} \sim Q \Lambda$. Expressed in terms of the Wilson coefficients defined in Sec. IV, they are given by

$$
\begin{aligned}
& T_{a}^{(+)}(E, \mu)=B_{1}^{(v)}(2 \hat{E}, \mu)+\frac{\left[E B_{2}^{(v)}(2 \hat{E}, \mu)+m_{B} B_{3}^{(v)}(2 \hat{E}, \mu)\right]}{\left(2 E-m_{B}\right)}, \\
& T_{a}^{(0)}(E, \mu)=B_{1}^{(v)}(2 \hat{E}, \mu)+\frac{\left[\left(m_{B}-E\right) B_{2}^{(v)}(2 \hat{E}, \mu)+m_{B} B_{3}^{(v)}(2 \hat{E}, \mu)\right]}{\left(m_{B}-2 E\right)}, \\
& T_{a}^{(T)}(E, \mu)=B_{1}^{(t)}(2 \hat{E}, \mu)-B_{2}^{(t)}(2 \hat{E}, \mu)-2 B_{3}^{(t)}(2 \hat{E}, \mu)+B_{4}^{(t)}(2 \hat{E}, \mu),
\end{aligned}
$$

where the dependence on $\hat{\omega}=2 \hat{E}$ is shown, and

$$
\begin{aligned}
& T_{b}^{(+)}(E, z, \mu)=B_{11}^{(v)}(2 \hat{E}, z, \mu)-\frac{E}{m_{B}} B_{12}^{(v)}(2 \hat{E}, x, \mu)-B_{13}^{(v)}(2 \hat{E}, z, \mu), \\
& T_{b}^{(0)}(E, z, \mu)=B_{11}^{(v)}(2 \hat{E}, z, \mu)-\frac{\left(m_{B}-E\right)}{m_{B}} B_{12}^{(v)}(2 \hat{E}, z, \mu)-B_{13}^{(v)}(2 \hat{E}, z, \mu), \\
& T_{b}^{(T)}(E, z, \mu)=B_{15}^{(t)}(2 \hat{E}, z, \mu)+B_{16}^{(t)}(2 \hat{E}, z, \mu)-B_{18}^{(t)}(2 \hat{E}, z, \mu),
\end{aligned}
$$

where $\hat{\omega}_{1}+\hat{\omega}_{2}=2 \hat{E}$ and the dependence on $z$ is induced from the $\hat{\omega}_{1}-\hat{\omega}_{2}$ dependence of the coefficients.

If we work at tree level in $J_{a, b}$ using Eq. (119) and also in $T_{a, b}$ then these coefficients are scale independent and satisfy $T_{a}^{(+, 0, T)}=T_{b}^{(+, 0)}=1$ and $T_{b}^{(T)}=0$. In this case if we take the ratios $f_{0} / f_{+}$and $f_{T} / f_{+}$and expand assuming that the $f^{F}$ terms are smaller than the $f^{N F}$ terms then our results agree with Ref. [19]. We note that using just the information in our factorization theorem that it is not clear that one wants to expand in this way since the $\mathrm{F}$ and NF terms could actually be similar in size as discussed in the introduction. The expectation from QCD sum rules is that the "soft" NF part of the form factors is larger than the "hard" F part [20]. 


\section{CONCLUSION}

The soft-collinear effective theory (SCET) allows a rich structure of allowed operators at higher orders in the expansion parameter $\lambda$. In contrast to simpler effective theories, the presence of fields $\left(\bar{n} \cdot A_{n}\right)$ and derivatives $\left(\bar{n} \cdot i D_{c}\right)$ scaling like $\lambda^{0}$, allows a continuum set of operators at any given order in $\lambda$. A similar situation is encountered in deep inelastic scattering, where an infinite number of operators of increasing dimension can contribute to the same order in $1 / Q$. In a generic process with energetic hadrons it is therefore important to have a well-defined procedure for organizing the structure of the soft-collinear operators at a given order in $\lambda$. This organization is provided by SCET.

In this paper we formulated a general prescription for constructing the most general ultrasoft-collinear operators appearing in the Lagrangian or in the matching of an external current at a given order in $\lambda$ but to all orders in $\alpha_{s}$. This was done by including constraints from collinear gauge invariance, the Dirac structure of the effective theory fields and reparameterization invariance. These conditions prove to be surprisingly predictive, and constrain not only the number of allowed operators, but also their functional dependence on label momenta.

For the case of the heavy-light currents, the constraints from the Dirac structure of the effective theory fields have been included at leading order in [2], and allow only 3 structures in the $v_{\perp}=0$ frame. Here we consider the more general case of an arbitrary heavy quark velocity $v$, which is necessary in order to have a set of operators which closes under reparameterization transformations.

At subleading order $O(\lambda)$ the Dirac constraints alone allow many more operators. In particular, in addition to 2-body operators $\left(\bar{\xi}_{n} W\right)_{\omega} \cdots h_{v}$, one has to include also 3-body currents of the form $\left(\bar{\xi}_{n} W\right)_{\omega_{1}} \cdots\left(W^{\dagger} i D W\right)_{\omega_{2}} \cdots h_{v}$. RPI constraints on a subset of the two body operators were previously considered in [10, 13], and it was shown their coefficients are fixed in terms of the coefficients of leading order currents. Here we extended the constraints to the full set of allowed two-body and three-body operators, and showed that type (II) RPI imposed severe constraints on the $\left(\omega_{1}, \omega_{2}\right)$ dependence of the latter. For example, the scalar current $\bar{q} b$ is matched at $O(\lambda)$ onto 8 general operators in the effective theory. After imposing all constraints, only one of these has a free Wilson coefficient, which has to be determined from a matching calculation. A similar reduction is obtained for the more complicated case of the vector/axial and tensor currents, for which one can write $(28,44)$ structures but only $(4,7)$ Wilson coefficients are not fixed by the symmetries of the effective theory.

In this paper we have focused on mixed usoft-collinear interactions, however for many exclusive heavy-to-light processes the final operators that are needed are of soft-collinear type as was the case for heavy-to-light form factors. In practice it appears simplest to derive collinear-soft interactions from the collinear-usoft ones using the two-stage matching technique, QCD $\rightarrow$ SCET $_{\mathrm{I}} \rightarrow \mathrm{SCET}_{\mathrm{II}}$, discussed in the proof of factorization for heavy-tolight decays Ref. [15]. The operators in this paper describe interactions in the intermediate $\mathrm{SCET}_{\mathrm{I}}$ theory. For exclusive processes such as $B \rightarrow D \pi[7]$ where the intermediate $p^{2} \sim Q \Lambda$ fluctuations in $\mathrm{SCET}_{\mathrm{I}}$ are responsible for inducing simple operators in $\mathrm{SCET}_{\mathrm{II}}$ the procedure used in Ref. [15] reduces to the one discussed in Ref. 41].

Note Added: In the final stages of this work, Ref. [17] appeared where a direct study of 
light-light soft-collinear operators was performed. The Wilson coefficients of these operators were determined by matching from QCD up to one-loop order, and both 2-body and 3-body operators were found to contribute. An intermediate theory with modes of momentum $p^{\mu} \sim Q\left(\lambda^{\prime}, 1, \lambda^{\prime}\right)$ as dynamical degrees of freedom was also considered. This appears similar in spirit to the QCD $\rightarrow \mathrm{SCET}_{\mathrm{I}} \rightarrow \mathrm{SCET}_{\mathrm{II}}$ construction used in Ref. [15], however in the intermediate $\mathrm{SCET}_{\mathrm{I}}$ theory we found that the dynamical collinear modes should have momentum scaling as $p^{\mu} \sim Q\left(\lambda^{\prime}, 1, \sqrt{\lambda^{\prime}}\right)$. Finally, reparameterization invariance constraints on soft-collinear operators were also discussed in Ref. [17], and were shown to constrain the form of certain Wilson coefficients.

\section{Acknowledgments}

We thank C. Bauer and T. Mehen for discussions. This work was supported in part by the Department of Energy under the grants NSF PHY-9970781 and DE-FG03-00-ER-41132. 
[1] C. W. Bauer, S. Fleming and M. Luke, Phys. Rev. D 63, 014006 (2001) [hep-ph/0005275].

[2] C. W. Bauer, S. Fleming, D. Pirjol and I. W. Stewart, Phys. Rev. D 63, 114020 (2001) [hep-ph/0011336].

[3] C. W. Bauer and I. W. Stewart, Phys. Lett. B 516, 134 (2001) [arXiv:hep-ph/0107001].

[4] C. W. Bauer, D. Pirjol and I. W. Stewart, Phys. Rev. D 65, 054022 (2002) [arXiv:hep$\mathrm{ph} / 0109045]$.

[5] For reviews R. L. Jaffe, [arXiv:hep-ph/9602236]; G. Sterman, TASI lectures 1995, [arXiv:hepph/9606312]; J.C. Collins, D.E. Soper, and G. Sterman in Perturbative Quantum Chromodynamics, Ed. by A. H. Mueller, World Scientific Publ., 1989, p. 1-93.

[6] S. J. Brodsky and G. P. Lepage, in Perturbative Quantum Chromodynamics, pp. 93-240; S. J. Brodsky, SLAC report SLAC-PUB-9281, arXiv:hep-ph/0208158.

[7] C. W. Bauer, D. Pirjol and I. W. Stewart, Phys. Rev. Lett. 87, 201806 (2001) [arXiv:hep$\mathrm{ph} / 0107002]$.

[8] C. W. Bauer, S. Fleming, D. Pirjol, I. Z. Rothstein and I. W. Stewart, Phys. Rev. D 66, 014017 (2002) [arXiv:hep-ph/0202088];

[9] C. W. Bauer, C. W. Chiang, S. Fleming, A. K. Leibovich and I. Low, Phys. Rev. D 64, 114014 (2001) [arXiv:hep-ph/0106316]; S. Descotes-Genon and C. T. Sachrajda, arXiv:hepph/0209216; E. Lunghi, D. Pirjol and D. Wyler, Nucl. Phys. B 649, 349 (2003) [arXiv:hep$\mathrm{ph} / 0210091]$.

[10] J. Chay and C. Kim, Phys. Rev. D 65, 114016 (2002) [arXiv:hep-ph/0201197].

[11] A. V. Manohar, T. Mehen, D. Pirjol and I. W. Stewart, Phys. Lett. B 539, 59 (2002) [arXiv:hep-ph/0204229].

[12] C. W. Bauer, D. Pirjol and I. W. Stewart, Phys. Rev. D 66, 054005 (2002). [arXiv:hep$\mathrm{ph} / 0205289]$.

[13] M. Beneke, A. P. Chapovsky, M. Diehl and T. Feldmann, Nucl. Phys. B 643, 431 (2002) [arXiv:hep-ph/0206152].

[14] T. Feldmann, arXiv:hep-ph/0209239.

[15] C. W. Bauer, D. Pirjol and I. W. Stewart, arXiv:hep-ph/0211069.

[16] R. K. Ellis, W. Furmansky and R. Petronzio, Nucl. Phys. B 207, 1 (1982); Nucl. Phys. B 212, 
29 (1983); R. L. Jaffe and M. Soldate, Phys. Rev. D 26, 49 (1982); A. V. Efremov and O. V. Teryaev, Yad. Phys. 39, 1517 (1984); I. V. Anikin and O. V. Teryaev, Phys. Lett. B 509, 95 (2001).

[17] R. J. Hill and M. Neubert, arXiv:hep-ph/0211018.

[18] A. Szczepaniak, E. M. Henley and S. J. Brodsky, Phys. Lett. B 243, 287 (1990); G. Burdman and J. F. Donoghue, Phys. Lett. B 270, 55 (1991); R. Akhoury, G. Sterman and Y. P. Yao, Phys. Rev. D 50, 358 (1994); H. n. Li and H. L. Yu, Phys. Rev. D 53, 2480 (1996) [arXiv:hepph/9411308]; H. n. Li, arXiv:hep-ph/0102013; H. n. Li, Phys. Rev. D 64, 014019 (2001) [arXiv:hep-ph/0012140]; T. Kurimoto, H. n. Li and A. I. Sanda, Phys. Rev. D 65, 014007 (2002) [arXiv:hep-ph/0105003]. S. Descotes-Genon and C. T. Sachrajda, Nucl. Phys. B 625, 239 (2002) [arXiv:hep-ph/0109260].

[19] M. Beneke and T. Feldmann, Nucl. Phys. B 592, 3 (2001).

[20] A. Khodjamirian, R. Ruckl, S. Weinzierl and O. I. Yakovlev, Phys. Lett. B 410, 275 (1997) [arXiv:hep-ph/9706303]. E. Bagan, P. Ball and V. M. Braun, Phys. Lett. B 417, 154 (1998) [arXiv:hep-ph/9709243]; P. Ball, V. M. Braun, Y. Koike and K. Tanaka, Nucl. Phys. B 529, 323 (1998) [arXiv:hep-ph/9802299]; P. Ball and V. M. Braun, Phys. Rev. D 58, 094016 (1998) [arXiv:hep-ph/9805422]; A. Khodjamirian, R. Ruckl, S. Weinzierl, C. W. Winhart and O. I. Yakovlev, Phys. Rev. D 62, 114002 (2000) [arXiv:hep-ph/0001297]; P. Ball, JHEP 9809, 005 (1998) [arXiv:hep-ph/9802394]; P. Ball and R. Zwicky, JHEP 0110, 019 (2001) [arXiv:hep-ph/0110115].

[21] V. L. Chernyak and I. R. Zhitnitsky, Nucl. Phys. B 345, 137 (1990).

[22] A. G. Grozin and M. Neubert, Phys. Rev. D 55, 272 (1997) [arXiv:hep-ph/9607366].

[23] J. Charles et al., Phys. Rev. D 60, 014001 (1999). [arXiv:hep-ph/9812358].

[24] M. J. Dugan and B. Grinstein, Phys. Lett. B 255, 583 (1991).

[25] U. Aglietti, G. Corbo and L. Trentadue, Int. J. Mod. Phys. A 14, 1769 (1999) [arXiv:hepph/9712237]; U. Aglietti and G. Corbo, Int. J. Mod. Phys. A 15, 363 (2000) [arXiv:hepph/9712242]; C. Balzereit, T. Mannel and W. Kilian, Phys. Rev. D 58, 114029 (1998) [arXiv:hep-ph/9805297]; see also the structure of interactions in Light-Cone QCD, for a recent review see S. Brodsky, arXiv:hep-ph/0208158.

[26] D. Politzer and M. Wise, Phys. Lett. B257, 399 (1991).

[27] J. B. Kogut and D. E. Soper, Phys. Rev. D1, 2901 (1970); J. D. Bjorken, J. B. Kogut and 
D. E. Soper, Phys. Rev. D3, 1382 (1971); H. Leutwyler, Nucl. Phys. B76, 413 (1974).

[28] M. E. Luke and A. V. Manohar, Phys. Lett. B 286, 348 (1992). [arXiv:hep-ph/9205228].

[29] A. V. Manohar and M. B. Wise, Cambridge Monogr. Part. Phys. Nucl. Phys. Cosmol. 10, 1 (2000).

[30] Y. Q. Chen, Phys. Lett. B 317, 421 (1993); M. Finkemeier, H. Georgi, and M. McIrvin, Phys. Rev. D 55, 6933 (1997); [arXiv:hep-ph/9701243]; A. V. Manohar, Phys. Rev. D 56, 230 (1997); [arXiv:hep-ph/9701294]; R. Sundrum, Phys. Rev. D 57, 331 (1998); [arXiv:hep-ph/9704256] F. Campanario and T. Mannel, Phys. Rev. D 65, 094017 (2002) [arXiv:hep-ph/0201136].

[31] M. Neubert, Phys. Lett. B 306, 357 (1993). [arXiv:hep-ph/9302269]

[32] M. E. Luke, A. V. Manohar and I. Z. Rothstein, Phys. Rev. D 61, 074025 (2000) [arXiv:hep$\mathrm{ph} / 9910209]$.

[33] J. Chay and C. Kim, arXiv:hep-ph/0205117.

[34] I. W. Stewart, arXiv:hep-ph/0207253.

[35] G. P. Korchemsky and G. Marchesini, Nucl. Phys. B 406, 225 (1993) [arXiv:hep-ph/9210281]; G. P. Korchemsky and A. V. Radyushkin, Nucl. Phys. B 283, 342 (1987). 